BULLETIN (New Series) OF THE

AMERICAN MATHEMATICAL SOCIETY

Volume 36, Number 4, Pages 433-488

S 0273-0979(99)00849-6

Article electronically published on August 19, 1999

\title{
MERITS AND DEMERITS OF THE ORBIT METHOD
}

\author{
A. A. KIRILLOV
}

Abstract. This survey is the expanded version of my talk at the AMS meeting in April 1997. I explain to non-experts how to use the orbit method, discuss its strong and weak points and advertise some open problems.

\section{Contents}

0 . Introduction

Merits versus Demerits

1. Geometry of coadjoint orbits

1.1. Coadjoint representation

1.2. Symplectic structure. The first approach

1.3. Symplectic structure. The second approach

1.4. Coadjoint invariant polynomials

1.5. The moment map and the universal property of coadjoint orbits

1.6. Some particular cases

2. The triumph of the orbit method: The case of solvable Lie groups

2.1. Lie groups and Lie algebras

2.2. The orbit method for nilpotent Lie groups

2.3. Exponential Lie groups

2.4. Solvable Lie groups

3. The first obstacle: Compact Lie groups

3.1. Geometry of coadjoint orbits for compact Lie groups

3.2. Borel-Weil-Bott theorem

3.3. The integral formula for characters

3.4. The multiplicity of a weight

3.5. Application to the symmetrization map

3.6. Intertwining operators

4. More troubles: Non compact semisimple groups

4.1. Principal and degenerate series

4.2. Discrete series

4.3. Complementary series

4.4. Metaplectic representation and its analogs

5. Beyond the Lie groups

5.1. $\mathfrak{p}$-adic and adelic groups

Received by the editors October 1998.

1991 Mathematics Subject Classification. Primary 22-XX, 20C35.

Key words and phrases. Unitary representations, Lie groups, coadjoint orbits, symplectic geometry, geometric quantization.

I wish to thank my students who taught me so much.

(C)1999 American Mathematical Society 
5.2. Finite groups

5.3. Infinite dimensional groups

5.4. Quantum groups and Poisson-Lie groups

5.5. Groups related to von Neumann factors of type II

6. Explanation of why the orbit method works

6.1. Mathematical argument

6.2. Physical argument

7. Byproducts, side effects and related topics

7.1. The moment map

7.2. Geometric quantization

7.3. Integrable systems

8. Some open problems and subjects for meditation

8.1. Functional dimension

8.2. Infinitesimal characters

8.3. Multiplicities and geometry

8.4. Complementary series

8.5. Finite groups

8.6. Infinite dimensional groups

References

\section{INTRODUCTION}

The idea behind the orbit method is the unification of harmonic analysis with symplectic geometry (and it can also be considered as a part of the more general idea of the unification of mathematics and physics). In fact, this is a post factum formulation. Historically, the orbit method was proposed in [K1] for the description of the unitary dual (i.e. the set of equivalence classes of unitary irreducible representations) of nilpotent Lie groups. It turned out that not only this problem but all other principal questions of representation theory-topological structure of the unitary dual, explicit description of the restriction and induction functors, character formulae, etc. - can be naturally answered in terms of coadjoint orbits. Moreover, the answers make sense for general Lie groups (and even beyond), though sometimes with more or less evident corrections. I mentioned in [K1] the possible applications of the orbit method to other types of Lie groups, ${ }^{1}$ but the realization of this program took much time and is still not accomplished despite the efforts of many authors. ${ }^{2}$

As usual, the faults of the method are continuations of its advantages. We quote briefly the most important ones.

\footnotetext{
${ }^{1}$ Unfortunately, this remark was omitted in the exposition of the article at the Bourbaki Seminar.

${ }^{2}$ Of course, I cannot list here all those who contributed to the development of the orbit method, but I certainly should mention the outstanding role of Bertram Kostant and Michel Duflo.
} 


\section{Merits versus Demerits}

1. Universality: the method works for Lie groups of any type over any field.

2. The rules are visual, easy to memorize and illustrate by a picture.

3. The method explains some facts which otherwise look mysterious.

4. It provides a great number of symplectic manifolds and Poisson commuting families of functions.

5. The method introduces two new fundamental notions: coadjoint orbits and moment maps.
1. The recipes are not accurately and precisely formulated.

2. Sometimes they are wrong and need corrections or modifications.

3. It could be difficult to transform this explanation into a rigorous proof.

4. Most completely integrable dynamical systems were discovered earlier by other methods.

5. The description of coadjoint orbits and their structure is sometimes not an easy problem.

The goals of this article are: first, to describe the essence of the orbit method for non-experts; second, to attract the younger generation of mathematicians to some old and still unsolved problems in representation theory where I believe the orbit method could be helpful. The level of exposition varies in different parts of the article so that both experts and beginners can find something interesting and useful. The interested reader can find most of the unexplained notions, terms and constructions elucidated in my book [K2] or in the survey [K3].

Of course, the bibliography is not complete. The AMS catalog of mathematical articles contains today about 500 papers where coadjoint orbits are mentioned and more than 2,000 papers on geometric quantization (which is the physical counterpart of the orbit method), so I included only papers quoted in the text.

In conclusion I want to express the hope that the orbit method becomes for my readers a source of thoughts and inspirations as it has been for me during the last thirty-five years.

\section{Geometry of condjoint orbits}

The main new object which appeared in the orbit method for the first time is the notion of a coadjoint orbit, i.e. an orbit of a Lie group $G$ in the space $\mathfrak{g}^{*}$ dual to $\mathfrak{g}=\operatorname{Lie}(G)$ where $G$ acts via the coadjoint representation. In this section we consider the geometry of coadjoint orbits and discuss the problem of their classification.

1.1. Coadjoint representation. Let $G$ be a Lie group, i.e. a smooth manifold endowed with a multiplication law which is a smooth map $G \times G \rightarrow G$ satisfying the group axioms. It is useful to have in mind the following example: $G$ is a subgroup and at the same time a smooth submanifold of $G L(n, \mathbb{R})$, the group of all invertible $n \times n$ matrices with real entries. Such groups $G$ we shall call matrix groups. ${ }^{3}$

Let $\mathfrak{g}=\operatorname{Lie}(G)$ be the tangent space $T_{e}(G)$ to $G$ at the unit point $e$. The group $G$ acts on itself by inner automorphisms: $A(g): x \mapsto g x g^{-1}$. The point $e$ is a fixed point of this action, and we get the derived map $A_{*}(g): \mathfrak{g} \rightarrow \mathfrak{g}$ which is usually denoted by $A d(g)$. The map $g \mapsto A d(g)$ is called the adjoint representation of

\footnotetext{
${ }^{3}$ This example is almost universal: any Lie group is locally isomorphic to a matrix group.
} 
$G$. In the matrix group case $\mathfrak{g}$ is a subspace of $\operatorname{Mat}_{n}(\mathbb{R})$, the space of all $n \times n$ real matrices, and the adjoint representation is simply matrix conjugation:

$$
\operatorname{Ad}(g) X=g X g^{-1}, X \in \mathfrak{g}, g \in G .
$$

Consider now the dual linear space to $\mathfrak{g}$ usually denoted by $\mathfrak{g}^{*}$. In the matrix case we can use the fact that $\operatorname{Mat}_{n}(\mathbb{R})$ has a bilinear form $\langle A, B\rangle=\operatorname{tr}(A B)$ which is invariant under conjugation. So, the space $\mathfrak{g}^{*}$ dual to the subspace $\mathfrak{g} \subset \operatorname{Mat}_{n}(\mathbb{R})$ can be identified with the quotient space $\operatorname{Mat}_{n}(\mathbb{R}) / \mathfrak{g}^{\perp}$ where the sign ${ }^{\perp}$ means the orthogonal complement with respect to $\langle$,$\rangle . In practice the latter space is often$ identified with some subspace $V \subset \operatorname{Mat}_{n}(\mathbb{R})$ which is transverse to $\mathfrak{g}^{\perp}$ and has the complementary dimension. Let $p$ be the projection of $\operatorname{Mat}_{n}(\mathbb{R})$ on $V$ parallel to $\mathfrak{g}^{\perp}$. Then the coadjoint representation $K$, which is dual to the adjoint representation defined above, can be written in the simple form

$$
K(g): F \mapsto p\left(g F g^{-1}\right) .
$$

Remark. If $V$ were invariant under $A d(G)$ (as we can assume for a semi-simple or reductive $\mathfrak{g}$ ), we could omit the projection $p$ in (1.1.1).

Example 1. Let $G$ be the group of upper triangular matrices from $G L(n, \mathbb{R})$. Then $\mathfrak{g}$ consists of all upper triangular matrices from $\operatorname{Mat}_{n}(\mathbb{R})$, and we can take for $V$ the space of all lower triangular matrices. The projection $p$ in this case sends any matrix to its "lower part" (i.e. replaces all entries above the main diagonal by zeros).

Example 2. Let $G=S O(n, \mathbb{R})$. Then $\mathfrak{g}$ consists of all skew-symmetric matrices from $\operatorname{Mat}_{n}(\mathbb{R})$. Here we can put $V=\mathfrak{g}$ and omit the projection $p$ in (1.1.1). The same is true for any reductive subgroup of $G L(n, \mathbb{R})$.

We also give the infinitesimal version of the coadjoint action:

$$
\left\langle K_{*}(X) F, Y\right\rangle=\langle F,-a d(X) Y\rangle=\langle F,[Y, X]\rangle
$$

which for matrix groups takes the form

$$
K_{*}(X) F=p([X, F]), X \in \mathfrak{g}, F \in \mathfrak{g}^{*} .
$$

1.2. Symplectic structure. The first approach. The most remarkable feature of the coadjoint representation is the fact that all coadjoint orbits possess a canonical $G$-invariant symplectic structure. This means that on each orbit $\Omega \subset \mathfrak{g}^{*}$ there is a canonically defined closed non-degenerate $G$-invariant differential 2 -form $\sigma$. Here we give a direct construction of this form.

First, we observe that an invariant differential form on a homogeneous manifold is uniquely determined by its value at a single point. Note that this value should be invariant under the action of the stabilizer of the point and subjected to no other restrictions. Thus, it is enough to specify the value of $\sigma$ at some point $F \in \Omega$.

Remark. For some groups (especially infinite dimensional; see 5.3) one can find an explicit formula for $\sigma$ only at a specially chosen point $F_{0}$ of a given orbit. This defines $\sigma$ uniquely but not explicitly.

Now let $\operatorname{Stab}(F)$ denote the stabilizer of $F \in \Omega$ and $\operatorname{stab}(F)$ be the Lie algebra of $\operatorname{Stab}(F)$. Consider the exact sequence

$$
0 \rightarrow \operatorname{stab}(F) \rightarrow \mathfrak{g} \rightarrow T_{F}(\Omega) \rightarrow 0
$$


which comes from the action of $G$ on $\Omega$. It allows us to identify the tangent space $T_{F}(\Omega)$ with the quotient of $\mathfrak{g}$ by $\operatorname{stab}(F)$. But there is a natural skew symmetric bilinear form on $\mathfrak{g}$ whose kernel is exactly $\operatorname{stab}(F)$, namely

$$
B_{F}(X, Y)=\langle F,[X, Y]\rangle .
$$

Indeed,

$$
\text { ker } \begin{aligned}
B_{F} & =\left\{X \in \mathfrak{g} \mid B_{F}(X, Y)=0 \forall Y \in \mathfrak{g}\right\} \\
& =\left\{X \in \mathfrak{g} \mid\left\langle K_{*}(X) F, Y\right\rangle=0 \forall Y \in \mathfrak{g}\right\}=\operatorname{stab}(F) .
\end{aligned}
$$

So, we can define the value $\sigma_{F}$ of $\sigma$ at the point $F$ by

$$
\sigma_{F}\left(K_{*}(X) F, K_{*}(Y) F\right)=B_{F}(X, Y) .
$$

The non-degeneracy and $G$-invariance of the constructed form $\sigma$ follow immediately from the definition. We could check directly that this form is closed by some more involved (though simple) computations (see e.g. [K2]), but instead we use the following observation.

For $F \in \Omega$ let $p_{F}$ denote the submersion $G \rightarrow \Omega: g \mapsto K(g) F$. Then $p_{F}^{*}(\sigma)$ is a left invariant 2-form on $G$. It turns out that this form is not only closed but exact. Namely, one can check that $p_{F}^{*}(\sigma)$ is the exterior derivative of the left invariant 1 -form

$$
\theta_{F}=\left\langle F, g^{-1} d g\right\rangle
$$

Since $p_{F}$ is a submersion, $p_{F}^{*}$ is injective. But $p_{F}^{*} d \sigma=d p_{F}^{*}(\sigma)=d^{2} \theta_{F}=0$. Hence, $\sigma$ is closed.

In general $\theta_{F}$ cannot be written as $p_{F}^{*}(\phi)$ for some 1 -form $\phi$ on $\Omega$, so we can't claim that $\sigma$ is exact.

Recall that a closed 2-form on a smooth orientable manifold is exact iff its integral over any 2-cycle vanishes. For future use we make the following

Definition. A coadjoint orbit $\Omega$ is integral if the canonical symplectic form $\sigma$ has the property

$$
\int_{C} \sigma \in \mathbb{Z} \text { for every geometric 2-cycle } \mathrm{C} \text { in } \Omega \text {. }
$$

The meaning of the integrality condition is revealed by the following

Proposition 1. The following are equivalent:

(i) An orbit $\Omega \subset \mathfrak{g}^{*}$ is integral.

(ii) There exists a line bundle over $\Omega$ with a Hermitian connection $\nabla$ such that

$$
\operatorname{curv}(\nabla)=2 \pi i \sigma .
$$

(iii) For any $F \in \Omega$ the 1-dimensional representation of the Lie algebra stab $F)$ given by

$$
X \mapsto 2 \pi i\langle F, X\rangle
$$

can be integrated to a unitary 1-dimensional representation of the Lie group $\operatorname{Stab}^{0}(F)$, the connected component of the identity in $\operatorname{Stab}(F)$. 
1.3. Symplectic structure. The second approach. We now discuss another way to introduce the canonical symplectic structure on coadjoint orbits. It is based on the notion of Poisson manifold, which is by definition a smooth manifold $M$ endowed with a bivector field $c=c^{i j} \partial_{i} \partial_{j}$ such that the Poisson brackets

$$
\left\{f_{1}, f_{2}\right\}=c^{i j} \partial_{i} f_{1} \partial_{j} f_{2}
$$

define a Lie algebra structure on $C^{\infty}(M) \cdot{ }^{4}$ Any symplectic manifold $(M, \sigma)$ has a canonical Poisson structure $c$ such that in some (and hence in every) local coordinate system the matrices $\left\|c^{i j}\right\|$ and $\left\|\sigma_{i j}\right\|$ are reciprocal to each other.

The main structure theorem about Poisson manifolds (see, e.g. [K3]) claims that any such manifold $(M, c)$ can be uniquely foliated by its symplectic leaves, i.e. represented as the disjoint union of submanifolds $\left\{M_{\alpha}\right\}_{\alpha \in A}$ such that the bivector $c$ admits a restriction $c_{\alpha}$ on each $M_{\alpha}$ and $\left(M_{\alpha}, c_{\alpha}\right)$ is a symplectic manifold for each $\alpha \in A$. In other words, the value of $\left\{f_{1}, f_{2}\right\}$ at the point $m \in M_{\alpha}$ is equal to $\left\{\left.f_{1}\right|_{M_{\alpha}},\left.f_{2}\right|_{M_{\alpha}}\right\}_{\alpha}(m)$ where $\{\cdot, \cdot\}_{\alpha}$ is the Poisson bracket on $M_{\alpha}$.

Now let $\mathfrak{g}$ be a Lie algebra with a basis $\left\{X_{i}\right\}_{1 \leq i \leq n}$ and structure constants $c_{i j}^{k}$. On the space $\mathfrak{g}^{*}$, dual to $\mathfrak{g}$, with coordinates given by the basis above, the bivector

$$
c=c_{i j}^{k} X_{k} \partial^{i} \wedge \partial^{j}
$$

defines a Poisson structure. (Indeed, to check the Jacobi identity it is sufficient to consider the Poisson brackets of linear functions on $\mathfrak{g}^{*}$. But it is clear from (1.3.1) that

$$
\left\{X_{i}, X_{j}\right\}=c_{i j}^{k} X_{k}
$$

i.e. the linear functions form a Lie algebra isomorphic to $\mathfrak{g}.)^{5}$

Proposition 2. The symplectic leaves of the Poisson manifold $\left(\mathfrak{g}^{*}, c\right)$ are exactly the coadjoint orbits.

For the proof see [K3] (or check it directly).

1.4. Coadjoint invariant polynomials. In this section we discuss the classification problem for coadjoint orbits. The first step in the solution of this problem is to find all invariants of the group action. We start with polynomial and rational invariants. It is known that for algebraic actions of complex algebraic groups on affine algebraic manifolds there are enough rational invariants to separate the orbits in the following sense:

The common level set of all rational invariants consists of a finite number of orbits, and a generic level is just one orbit.

Moreover, each rational invariant can be written in the form $R=\frac{P}{Q}$ where $P$ and $Q$ are relatively invariant polynomials of the same weight (i.e. there is a homomorphism $\lambda: G \rightarrow \mathbb{C}^{\times}$such that both $P$ and $Q$ under the action of a $g \in G$ are multiplied by $\lambda(g)$ ). In particular, for nilpotent and semisimple groups which have no non-trivial rational homomorphisms to $\mathbb{C}^{\times}$, all rational invariants are quotients of polynomial invariants.

For real algebraic groups the common level sets of invariants could be split into finitely many connected components which are not separated by rational invariants.

\footnotetext{
${ }^{4}$ This imposes on $c$ the condition $[c, c]=0$ where $[$,$] is the so-called Schouten bracket operation$ on polyvector fields.

${ }^{5}$ As A. Weinstein clarified recently, this Poisson structure was known already to Sophus Lie. Being out of use for a long time, it was rediscovered by F. A. Berezin in 1968.
} 
(Compare with the geometry of quadrics in a real affine plane. The two branches of a hyperbola provide the most visual example.)

The general scheme for the construction of invariants of a group $G$ acting on a space $X$ is the following. Suppose we can construct a subset $S \subset X$ whose intersection with (almost) every orbit is a unique point. Any invariant function on $X$ defines by restriction a function on $S$. Conversely, any function on $S$ can be canonically extended to a $G$-invariant function defined (almost everywhere) on $X$. If $S$ is a smooth (resp. algebraic, rational, etc.) submanifold, then we get the information about smooth (resp. algebraic, rational, etc.) invariants.

Warning. While restriction to $S$ usually preserves the nice properties of invariants, the extension procedure does not. E.g., the extension of a polynomial function on $S$ could only be rational on $X$ (see Example 4 below).

Example 3. Let $G=G L(n, \mathbb{R})$ act on $X=\operatorname{Mat}_{n}(\mathbb{R})$ by conjugation. Let $S$ be the affine subspace consisting of matrices of the form

$$
\left(\begin{array}{ccccc}
0 & 1 & 0 & \cdots & 0 \\
0 & 0 & 1 & \cdots & 0 \\
\cdot & \cdot & \cdot & \cdots & \cdot \\
0 & 0 & 0 & \cdots & 1 \\
c_{n} & c_{n-1} & c_{n-2} & \cdots & c_{1}
\end{array}\right) .
$$

One can check that $S$ intersects almost all conjugacy classes in exactly one point. ${ }^{6}$ It follows that all polynomial invariants form an algebra $\mathbb{R}\left[c_{1}, c_{2}, \cdots, c_{n}\right]$. Indeed, every polynomial invariant restricted to $S$ becomes a polynomial in $c_{1}, c_{2}, \cdots, c_{n}$. On the other hand, all $c_{i}$ 's admit extensions as invariant polynomials on $\operatorname{Mat}_{n}(\mathbb{R})$ - they coincide up to sign with the coefficients of the characteristic polynomial $P_{A}(\lambda)=\operatorname{det}(A-\lambda \cdot 1)$.

There is a nice generalization of this example to all semisimple Lie algebras due to B. Kostant.

Example 4. Let $N_{+}$(resp. $N_{-}$) be the subgroup of strictly upper (resp. lower) triangular matrices from $G L(n, \mathbb{R})$. The group $G=N_{+} \times N_{-}$acts on $X=\operatorname{Mat}_{n}(\mathbb{R})$ :

$$
g=\left(n_{+}, n_{-}\right): A \mapsto n_{+} \cdot A \cdot n_{-}^{-1} .
$$

Take the subspace of diagonal matrices as $S$. Then almost all $G$-orbits intersect $S$ in a single point. But now, polynomial functions on $S$ extend to rational invariant functions on $X$. Namely, let $\Delta_{k}(A)$ denote the principal minor of order $k$ for a matrix $A$. It is a $G$-invariant polynomial on $X$, and the $k$-th diagonal element on $S$ extends as a rational function $\Delta_{k}(A) / \Delta_{k-1}(A)$ on $X$.

In the case of the coadjoint action the polynomial and rational invariants play an important role in representation theory due to their connection with so-called infinitesimal characters (see the next section). Here we remark only that smooth $K(G)$-invariants on $\mathfrak{g}^{*}$ form the center of the Lie algebra $C^{\infty}\left(\mathfrak{g}^{*}\right)$ with respect to Poisson brackets. Indeed, this center consists of functions $f$ such that

$$
c_{i j}^{k} X_{k} \partial^{i} f=0 \quad \forall j \in[1, n] .
$$

But this means exactly that $f$ is annihilated by all Lie vector fields $K_{*}\left(X_{j}\right)$, $1 \leq j \leq n$, hence is $K(G)$-invariant.

\footnotetext{
${ }^{6}$ Geometrically this means that for almost all operators $A \in \operatorname{Mat}_{n}(\mathbb{R})$ there exists a cyclic vector $\xi$, i.e. such that the vectors $\xi, A \xi, A^{2} \xi, \cdots, A^{n-1} \xi$ form a basis in $\mathbb{R}^{n}$.
} 
1.5. The moment map and the universal property of coadjoint orbits. We have seen that any coadjoint orbit is a homogeneous symplectic manifold. The converse is "almost true": up to some algebraic and topological corrections (see [K2] for details) any homogeneous symplectic manifold is a coadjoint orbit. This theorem looks more natural in the context of Poisson manifolds. Later on we assume that $G$ is a connected Lie group.

Let us define a Poisson $G$-manifold as a pair $\left(M, f_{(\cdot)}^{M}\right)$ where $M$ is a Poisson manifold with an action of $G$ and $f_{(\cdot)}^{M}: \mathfrak{g} \rightarrow C^{*}(M): X \mapsto f_{X}^{M}$ is a Lie algebra homomorphism such that the following diagram is commutative:

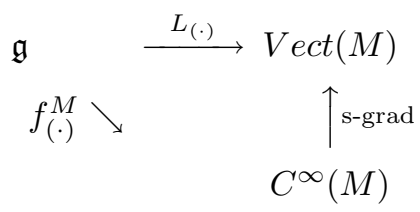

Here $L_{X}$ is the Lie field on $M$ associated with $X \in \mathfrak{g}$, and s-grad $(f)$ denotes the skew gradient of a function $f$, i.e. the vector field on $M$ such that

$$
\text { s-grad }(f) g=\{f, g\} \quad \text { for all } g \in C^{\infty}(M) .
$$

For a given Lie group $G$ the collection of all Poisson $G$-manifolds forms the category $\mathcal{P}(G)$ where a morphism $\alpha:\left(M, f_{(\cdot)}^{M}\right) \rightarrow\left(N, f_{(\cdot)}^{N}\right)$ is a smooth map from $M$ to $N$ which preserves the Poisson brackets: $\left\{\alpha^{*}(\phi), \alpha^{*}(\psi)\right\}=\alpha^{*}(\{\phi, \psi\})$ and makes the following diagram commutative:

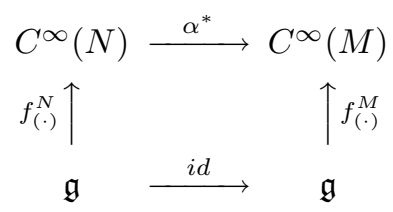

Observe that the last condition implies that $\alpha$ commutes with the $G$-action.

An important example of a Poisson $G$-manifold is the space $\left(\mathfrak{g}^{*}, c\right)$ considered in subsection 1.3. Here the map $\mathfrak{g} \rightarrow C^{\infty}\left(\mathfrak{g}^{*}\right)$ is defined by $f_{X}^{\mathfrak{g}^{*}}(F)=\langle F, X\rangle$.

Theorem 1. The Poisson $G$-manifold $\left(\mathfrak{g}^{*}, c\right)$ is a universal attracting object in the category $\mathcal{P}(G)$.

This means that for any object $\left(M, f_{(\cdot)}^{M}\right)$ there exists a unique morphism $\mu$ : $\left(M, f_{(\cdot)}^{M}\right) \rightarrow\left(\mathfrak{g}^{*}, f_{(\cdot)}^{\mathfrak{g}^{*}}\right)$, namely, the so-called moment map defined by

$$
\langle\mu(m), X\rangle=f_{X}^{M}(m) .
$$

Proof. Direct corollary of property (1.5.3) of a morphism in the category $\mathcal{P}(G)$.

We observe that for a homogeneous Poisson $G$-manifold its image under the moment map $\mu$ is necessarily a coadjoint orbit. Moreover, the transitivity of a $G$ action implies that $\mu$ is locally a diffeomorphism. Hence, any homogeneous Poisson $G$-manifold is a covering of a coadjoint orbit. I call this the universal property of coadjoint orbits. 
1.6. Some particular cases. For most "classical" (or "natural") groups the classification of coadjoint orbits is equivalent to one or another already known problems. In some cases, especially for infinite dimensional groups, new interesting geometric and analytic problems arise. We discuss here only three examples. Some others will appear below.

Example 5. Let $G=G L(n, \mathbb{R})$. Then $\mathfrak{g}=\operatorname{Mat}_{n}(\mathbb{R})$ possesses an $A d(G)$-invariant bilinear form (cf. 1.1) $\langle A, B\rangle=\operatorname{tr}(A B)$ so that the coadjoint representation is equivalent to the adjoint one. The classification of coadjoint orbits is therefore nothing but the problem of classification of matrices up to similarity.

Example 6. Let $M$ be a compact, smooth, simply connected 3-dimensional manifold with a given volume form vol. Let $G=\operatorname{Diff}(M, v o l)$ be the group of diffeomorphisms of $M$ which preserve the volume form.

The role of the Lie algebra $\mathfrak{g}=\operatorname{Lie}(G)$ is played by the space $\operatorname{Vect}(M, \mathrm{vol})$ of all smooth, divergence-free vector fields on $M$. We recall that the divergence of a vector field $\xi$ w.r.t. a volume form $v o l$ is a function $\operatorname{div} \xi$ on $M$ such that $L_{\xi}(v o l)=\operatorname{div} \xi \cdot v o l$. Here $L_{\xi}$ is the Lie derivative along the field $\xi$. Using the well known equality

$$
L_{\xi}=d \circ i_{\xi}+i_{\xi} \circ d
$$

we obtain that $i_{\xi}$ vol $=d \theta_{\xi}$ where $\theta_{\xi}$ is some 1 -form on $M$ defined modulo exact forms (differentials of functions). Now any smooth map $K: S^{1} \rightarrow M$ defines a linear functional $F_{K}$ on $\mathfrak{g}$ :

$$
F_{K}(\xi)=\int_{S^{1}} K^{*}\left(\theta_{\xi}\right)
$$

(It is clear that adding to $\theta_{\xi}$ a differential of a function does not change the value of the integral.) Moreover, the functional $F_{K}$ does not change if we reparametrize $S^{1}$ so that the orientation is preserved. In other words, it depends only on the oriented curve $K\left(S^{1}\right)$.

We see that the classification of coadjoint orbits for this group contains as a particular case the classification of oriented knots in $M$ up to a volume-preserving isotopy.

Example 7. Let Diff $+\left(S^{1}\right)$ denote the group of orientation preserving diffeomorphisms of the circle and $G$ be its only non-trivial central extension. This example will be considered more thoroughly in 5.3.3. Here we note only that the classification of coadjoint orbits for this group is equivalent to each of the following apparently unrelated problems.

1. Consider the second order differential equation

$$
L y:=c y^{\prime \prime}+p(x) y=0 .
$$

If we change the independent variable: $x \mapsto \phi(t)$ and at the same time change the unknown function $y \mapsto y \circ \phi \cdot\left(\phi^{\prime}\right)^{-\frac{1}{2}}$, the equation $L y=0$ goes to the equation $\tilde{L} \tilde{y}=0$ of the same form but with new coefficient

$$
\tilde{p}=p \circ \phi \cdot\left(\phi^{\prime}\right)^{2}+c S(\phi) \text { where } S(\phi)=\frac{\phi^{\prime \prime \prime}}{\phi^{\prime}}-\frac{3}{2}\left(\frac{\phi^{\prime \prime}}{\phi^{\prime}}\right)^{2} .
$$

Assume now that the coefficient $p(x)$ is $2 \pi$-periodic and the function $\phi(t)$ has the property $\phi(t+2 \pi)=\phi(t)+2 \pi$. The problem is to classify the equations (1.6.2) up to transformations (1.6.3). (For more details see 5.3.3.) 
2. Let $G$ be the simply-connected covering of the group $S L(2, \mathbb{R}),{ }^{7}$ and let $A$ be the group of all automorphisms of $G$. The problem is to classify elements of $G$ up to the action of $A$.

3. The locally projective structure on the oriented circle $S^{1}$ is defined by a covering of $S^{1}$ by charts $\left\{U_{\alpha}\right\}_{\alpha \in A}$ with local parameter $t_{\alpha}$ on $U_{\alpha}$ such that the transition functions $\phi_{\alpha \beta}$ are fractional linear and orientation preserving. (This means that $t_{\alpha}=\frac{a t_{\beta}+b}{c t_{\beta}+d}, a d-b c>0$.)

The problem is to classify the locally projective structures on $S^{1}$ up to the action of $\operatorname{Diff}_{+}\left(S^{1}\right)$.

\section{The triumph of the orbit method: The case of solvable Lie groups}

2.1. Lie groups and Lie algebras. The tangent space $T_{x}(X)$ can be considered as a linear approximation to a non-linear manifold $X$. In the case of Lie groups this has much deeper meaning. We recall that any connected and simply connected Lie group $G$ can be uniquely reconstructed from its Lie algebra $\mathfrak{g}=\operatorname{Lie}(G)=T_{e}(G)$.

Moreover, the exponential map exp : $\mathfrak{g} \rightarrow G$ is defined ${ }^{8}$ such that for any local homomorphism $\rho$ of one Lie group to another we have the commutative diagram:

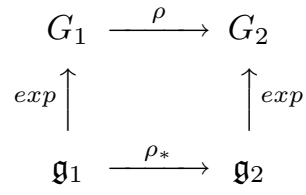

where $\rho_{*}$ is the derivative of $\rho$ at the unit point $e \in G$. So, the category of simply connected Lie groups is isomorphic to the category of Lie algebras.

The orbit method is also a kind of approximation, and the nicer the relation between a Lie group and its Lie algebra, the better it works.

A Lie group $G$ is called exponential if the map exp : $\mathfrak{g} \rightarrow G$ is a diffeomorphism. It is known that the class of exponential Lie groups lies strictly between nilpotent and solvable groups. In particular, no semisimple group is exponential. On the other side, the group $T_{+}(n, \mathbb{R})$ of all upper triangular real matrices with positive diagonal elements is exponential. It is worth mentioning that the property of being exponential is inherited by closed connected subgroups and by semi-direct extensions.

Now we introduce notations and assumptions and also recall some basic facts.

$G$ - a connected and simply connected Lie group, $\mathfrak{g}=\operatorname{Lie}(G)$ - its Lie algebra, $\mathfrak{g}^{*}$ - the dual space.

$P\left(\mathfrak{g}^{*}\right)$ - the naturally graded algebra of polynomial functions on $\mathfrak{g}^{*}$; we identify it with the symmetric algebra $S(\mathfrak{g})$ via the map $\mathfrak{g} \ni X \mapsto 2 \pi i\langle\cdot, X\rangle \in P^{1}\left(\mathfrak{g}^{*}\right)$.

$Y(\mathfrak{g})=P\left(\mathfrak{g}^{*}\right)^{G}$ - the algebra of $G$-invariant polynomials on $\mathfrak{g}^{*}$.

$U(\mathfrak{g})$ - the universal enveloping algebra of the Lie algebra $\mathfrak{g}$ (algebraically, it is an associative algebra over $\mathbb{C}$ generated by $\mathfrak{g}$ with relations $X \cdot Y-Y \cdot X=[X, Y]$; analytically, $U(\mathfrak{g})$ can be viewed as the algebra of all left invariant differential operators on $G$ so that elements of $\mathfrak{g}$ are realized by the infinitesimal right shifts). For any representation $T$ of $G$ in a linear topological space $V$ one can define the

\footnotetext{
${ }^{7}$ This group has no faithful finite dimensional linear representations. The simplest non-linear representation is the action of this group on $\mathbb{R}$ which covers the fractional-linear action of $S L(2, \mathbb{R})$ on $P^{1}(\mathbb{R}) \simeq S^{1}$.

${ }^{8}$ For matrix groups the exponential map is given by the standard formula: $\exp X=\sum_{n=0}^{\infty} \frac{X^{n}}{n !}$.
} 
corresponding representation $T^{\infty}$ of $U(\mathfrak{g})$ in the space $V^{\infty}$ of smooth vectors in $V$ (i.e. such vectors $\xi \in V$ for which the map $G \ni g \mapsto T(g) \xi \in V$ is of class $C^{\infty}$ ).

$Z(\mathfrak{g})$ - the center of $U(\mathfrak{g})$ (its elements, viewed as differential operators on $G$, are left and right invariant; they are called Laplace or Casimir operators).

The symmetrization map sym $: S(\mathfrak{g}) \rightarrow U(\mathfrak{g})$ is a linear map defined on monomials by

$$
\operatorname{sym}\left(X_{1} X_{2} \cdots X_{k}\right)=\frac{1}{k !} \sum_{\sigma \in S_{k}} \operatorname{sgn}(\sigma) X_{\sigma(1)} X_{\sigma(2)} \cdots X_{\sigma(k)} \text { for } X_{1}, X_{2}, \cdots, X_{k} \in \mathfrak{g} .
$$

In particular, for any $X \in \mathfrak{g}$ we have $\operatorname{sym}\left(X^{n}\right)=X^{n} \in U(\mathfrak{g})$.

This map is $G$-covariant and bijective. Using the above identification of $S(\mathfrak{g})$ and $P\left(\mathfrak{g}^{*}\right)$ we get a $G$-covariant linear isomorphism $U(\mathfrak{g}) \rightarrow P\left(\mathfrak{g}^{*}\right): A \mapsto p_{A}$ which maps the subspace $Z(\mathfrak{g})$ onto $Y(\mathfrak{g})$.

$\widehat{G}$ - the unitary dual of the group $G$, i.e. the set of (equivalence classes of) unitary irreducible representations (unirreps for short) of $G$.

$H$ - a closed connected subgroup of $G, \mathfrak{h}$ - its Lie algebra, $\mathfrak{h}^{*}$ - the dual space.

$p$ - the natural projection of $\mathfrak{g}^{*}$ onto $\mathfrak{h}^{*}$ dual to the inclusion $\mathfrak{h} \hookrightarrow \mathfrak{g}$.

$\mathcal{O}(G)=\mathfrak{g}^{*} / G$ - the set of coadjoint orbits of $G$.

$\operatorname{Res}_{H}^{G} T$ - the restriction to $H$ of the representation $T$ of $G$.

$\operatorname{Ind}_{H}^{G} S$ - the representation of $G$ induced by the representation $S$ of $H$.

$T_{\Omega}$ - the representation associated to the coadjoint orbit $\Omega$.

A subalgebra $\mathfrak{h} \subset \mathfrak{g}$ is called subordinate to a functional $F \in \mathfrak{g}^{*}$ if $\left.F\right|_{[\mathfrak{h}, \mathfrak{h}]}=0$; in this case the formula

$$
U_{F, H}(\exp X):=e^{2 \pi i\langle F, X\rangle}, X \in \mathfrak{h},
$$

defines a 1-dimensional unitary representation $U_{F, H}$ of the subgroup $H=\exp (\mathfrak{h})$.

The generalized character of a representation $T$ of $G$ is the distribution $\chi_{T}$ on $G$ which is defined as follows. Let $C_{c}^{\infty}(G)$ denote the space of smooth compactly supported functions on $G$. For $\phi \in C_{c}^{\infty}(G)$ we define $T_{\phi}:=\int_{G} \phi(g) T(g) d g$ where $d g$ is the Haar measure on $G$. It is known that for all unirreps of nilpotent or semisimple Lie groups this operator belongs to the trace class. We put

$$
\left\langle\chi_{T}, \phi\right\rangle:=\operatorname{tr} T_{\phi} .
$$

The infinitesimal character of a unirrep $T$ is by definition the homomorphism $\lambda_{T}: Z(\mathfrak{g}) \rightarrow \mathbb{C}$ such that for any $A \in Z(\mathfrak{g})$ we have

$$
T^{\infty}(A)=\lambda_{T}(A) \cdot 1 \text {. }
$$

It also can be defined by

$$
T(A \phi)=\lambda_{T}(A) \cdot T(\phi) \text { for } \phi \in C_{c}^{\infty}(G) .
$$

Here in the left hand side $A \in Z(\mathfrak{g})$ is considered as a differential operator on $G$.

2.2. The orbit method for nilpotent Lie groups. This is the ideal situation where the orbit method works most perfectly. We shall formulate the results in the form of a "user's guide", where practical instructions are given on how to get answers to ten basic questions of representation theory. Almost all results in this section were obtained in [K1]; the homeomorphism of $\widehat{G}$ and $\mathcal{O}(G)$ was proved later in $[\mathrm{B}]$. 
Recall once again that these simple rules are applicable only for simply connected nilpotent groups. For groups of general type we formulate later the "ten amendments" to these rules.

What do you want

1. Describe the unitary dual $\widehat{G}$ as a topological space.

2. Construct the unirrep $T_{\Omega}$ associated to the orbit $\Omega \in \mathfrak{g}^{*}$.

3. Describe the spectrum of $\operatorname{Res}_{H}^{G} T_{\Omega}$.

4. Describe the spectrum of $\operatorname{Ind}_{H}^{G} S_{\Omega}$.

5. Describe the spectrum of the tensor product $T_{\Omega_{1}} \otimes T_{\Omega_{2}}$.

6. Compute the generalized character of $T_{\Omega}$.

7. Compute the infinitesimal character of $T_{\Omega}$.

8. What is the relation between $T_{\Omega}$ and $T_{-\Omega}$ ?

9. Find the functional dimension of $T_{\Omega}$.

10. Compute the Plancherel measure $\mu$ on $\widehat{G}$.

\section{USER'S GUIDE}

What you have to do

Take the space $\mathcal{O}(G)$ of coadjoint orbits with the quotient topology.

Choose a point $F \in \Omega$, take a subalgebra

$\mathfrak{h}$ of maximal dimension subordinate

to $F$ and put $T_{\Omega}=\operatorname{Ind}_{H}^{G} U_{F, H}$.

Take the projection $p(\Omega)$ and split it into $H$-orbits.

Take the $G$-saturation of $p^{-1}(\Omega)$ and split it into $G$-orbits.

Take the arithmetic sum $\Omega_{1}+\Omega_{2}$ and split it into orbits.

$\operatorname{tr} T_{\Omega}(\exp X)=\int_{\Omega} e^{2 \pi i\langle F, X\rangle+\sigma}$ or

$\left\langle T_{\Omega}, \phi\right\rangle=\int_{\Omega} \tilde{\phi}(F) e^{\sigma}$.

For $A \in Z(\mathfrak{g})$ take the value

of $p_{A}$ on the orbit $\Omega$.

They are contragradient (dual)

to each other.

It is equal to $\frac{1}{2} \operatorname{dim} \Omega$.

The measure on $\mathcal{O}(G)$ arising when the Lebesgue measure on $\mathfrak{g}^{*}$ is decomposed into canonical measures on coadjoint orbits.

We give below some worked examples to illustrate how this guide works. Other instructive examples can be found in [K3], Chapter $7, \S 2$.

Example 1. Let $\mathfrak{g}$ be the only 4-dimensional nilpotent Lie algebra which does not split into a direct sum of ideals. ${ }^{9}$ It has a basis $\left\{X_{1}, X_{2}, X_{3}, Y\right\}$ with the commutation relations

$$
\left[X_{i}, X_{j}\right]=0,\left[Y, X_{i}\right]=X_{i-1}, i=2,3 .
$$

The matrix realization of $\mathfrak{g}$ looks as follows:

$$
\sum_{i=1}^{3} a_{i} X_{i}+b Y=\left(\begin{array}{cccc}
0 & b & 0 & a_{1} \\
0 & 0 & b & a_{2} \\
0 & 0 & 0 & a_{3} \\
0 & 0 & 0 & 0
\end{array}\right) .
$$

The general group element is

$$
g\left(\alpha_{1}, \alpha_{2}, \alpha_{3}, \beta\right)=\left(\begin{array}{cccc}
1 & \beta & \frac{\beta^{2}}{2} & \alpha_{1} \\
0 & 1 & \beta & \alpha_{2} \\
0 & 0 & 1 & \alpha_{3} \\
0 & 0 & 0 & 1
\end{array}\right) .
$$

\footnotetext{
${ }^{9}$ Representations of this Lie algebra are used in the theory of an anharmonic oscillator and were first described in [D1].
} 
The element $F \in \mathfrak{g}^{*}$ with coordinates $\left\{X_{1}, X_{2}, X_{3}, Y\right\}$ can be written as a lower triangular matrix

$$
F=\left(\begin{array}{cccc}
0 & 0 & 0 & 0 \\
Y & 0 & 0 & 0 \\
0 & 0 & 0 & 0 \\
X_{1} & X_{2} & X_{3} & 0
\end{array}\right)
$$

and the projection $p: \operatorname{Mat}_{4}(\mathbb{R}) \rightarrow \mathfrak{g}^{*}$ has the form:

$$
p\left(\left\|A_{i j}\right\|\right)=\left(\begin{array}{cccc}
0 & 0 & 0 & 0 \\
A_{21}+A_{32} & 0 & 0 & 0 \\
0 & 0 & 0 & 0 \\
A_{41} & A_{42} & A_{43} & 0
\end{array}\right) .
$$

Direct computation shows that the coadjoint action is

$$
\begin{aligned}
& K\left(g\left(\alpha_{1}, \alpha_{2}, \alpha_{3}, \beta\right)\right)\left(X_{1}, X_{2}, X_{3}, Y\right)= \\
& \left(X_{1}, X_{2}-\beta X_{1}, X_{3}-\beta X_{2}+\frac{\beta^{2}}{2} X_{1}, Y+\alpha_{2} X_{1}+\alpha_{3} X_{2}-\beta \alpha_{3} X_{1}\right) .
\end{aligned}
$$

We see immediately that $p_{1}(F)=X_{1}$ is an invariant polynomial on $\mathfrak{g}^{*}$ (indeed, the element $X_{1}$ belongs to the center of $\mathfrak{g}$ ). But this cannot be the only invariant, because the generic orbit cannot have the dimension $3 .^{10}$

To find another invariant, let's consider the plane $X_{2}=0, Y=0$. It is clear from (2.2.2) that almost all orbits meet this plane. An easy computation shows that for the orbit of $\left(X_{1}, X_{2}, X_{3}, Y\right)$ the intersection point has the coordinates $\left(X_{1}, 0, X_{3}-\frac{X_{2}^{2}}{2 X_{1}}, 0\right)$. So, the desired polynomial invariant is $p_{2}=2 X_{1} X_{3}-X_{2}^{2}$.

Consider the common level set of these two invariants given by

$$
p_{1}(F)=c_{1}, \quad p_{2}(F)=c_{2} .
$$

We leave it to the reader to check that for $c_{1} \neq 0$ this level set is a single orbit. For $c_{1}=0, c_{2}<0$ it splits into two separate orbits distinguished by the sign of $X_{2}$; for $c_{1}=0, c_{2}>0$ the level is empty; and finally for $c_{1}=c_{2}=0$ it splits into 0 -dimensional orbits (fixpoints of the coadjoint action).

The final description of the topological space $\widehat{G}$ looks as follows. Take a real plane $\mathbb{R}_{c_{1}, c_{2}}^{2}$, delete the line $c_{1}=0$, and glue in two points instead of each point of the deleted ray $c_{1}<0$ and a whole 2-plane instead of the deleted origin.

Example 2. Let us derive the explicit formulae for unirreps of the group $G$ from Example 1.

The 1-dimensional representation associated with the one-point orbit $\{(0,0, a, b)\}$ is

$$
U_{a, b}\left(g\left(\alpha_{1}, \alpha_{2}, \alpha_{3}, \beta\right)\right)=e^{2 \pi i\left(a \alpha_{3}+b \beta\right)} .
$$

All the other representations correspond to 2-dimensional orbits. They are infinite dimensional and have the functional dimension one (see rule 9). Roughly speaking, the latter statement means that the representation is naturally realized in a space of functions of one variable. ${ }^{11}$ In our case, according to rule 2 , each of

\footnotetext{
${ }^{10}$ Recall that coadjoint orbits are even dimensional.

${ }^{11}$ For a more detailed discussion of the notion of functional dimension we refer to [K4], Part II. See also the discussion in Chapter 8.
} 
these representations is induced from a 1-dimensional representation of the appropriate 3-dimensional subgroup. In fact, for all the cases we can take the subgroup $A=\exp \mathfrak{a}$ where $\mathfrak{a}$ is spanned by $X_{1}, X_{2}, X_{3}$. (It is clear that $\mathfrak{a}$, being abelian, is subordinate to any functional and has the maximal possible dimension.)

Now we recall the standard procedure (described in detail e.g. in [K2]) to form a unitary induced representation $\operatorname{Ind}_{A}^{G} U$. For a moment we assume that the homogeneous space $X=A \backslash G$ admits a $G$-invariant measure $\mu$. In case of nilpotent Lie groups such a measure always exists and is unique up to a constant factor. In the general case the construction below needs a slight modification (see [K2]).

In fact, there are two equivalent procedures: one is more convenient in theoretical questions, and the other in computations.

1. Consider the space $L(G, A, U)$ of complex valued functions $\phi$ on $G$ satisfying

$$
\phi(a g)=U(a) \phi(g) \text {, for all } a \in A, g \in G \text {. }
$$

The group $G$ acts on $L(G, A, U)$ by right shifts:

$$
\left(T\left(g_{1}\right) \phi\right)(g)=\phi\left(g g_{1}\right) \text {. }
$$

One can introduce an inner product in $L(G, A, U)$ so that $T$ becomes a unitary representation. This is the first way to define $\operatorname{Ind}_{A}^{G} U$.

2. Consider the right coset space $X=A \backslash G$, and choose a representative $s(x) \in$ $G$ for each coset $x \in X$. Then each group element can be uniquely written as $g=a \cdot s(x), a \in A, x \in X$. Apply this to the element $s(x) \cdot g$, and write

$$
s(x) \cdot g=a \cdot s(y), \text { where } a=a(x, g) \in A, y=y(x, g) \in X .
$$

The induced representation $T=\operatorname{Ind}_{A}^{G} U$ acts by definition on the Hilbert space $\mathcal{H}=L^{2}(X, \mu)$ via

$$
(T(g) f)(x)=U(a(x, g)) \cdot f(y(x, g)) .
$$

One can check that representations (2.2.6) and (2.2.8) are in fact equivalent. Namely, to a function $\phi \in L(G, A, U)$ there corresponds the function $f=\phi \circ s \in \mathcal{H}$.

In our special case it is convenient to identify the measure space $(X, \mu)$ with $\left(\mathbb{R}^{1}, d x\right)$ and take the element $\exp x Y$ as $s(x), x \in \mathbb{R}^{1}$.

Next we have to solve the equation (2.2.6), which in our case takes the form

$$
\left(\begin{array}{cccc}
1 & x & \frac{x^{2}}{2} & 0 \\
0 & 1 & x & 0 \\
0 & 0 & 1 & 0 \\
0 & 0 & 0 & 1
\end{array}\right) \cdot\left(\begin{array}{cccc}
1 & \beta & \frac{\beta^{2}}{2} & \alpha_{1} \\
0 & 1 & \beta & \alpha_{2} \\
0 & 0 & 1 & \alpha_{3} \\
0 & 0 & 0 & 1
\end{array}\right)=\left(\begin{array}{cccc}
1 & 0 & 0 & a_{1} \\
0 & 1 & 0 & a_{2} \\
0 & 0 & 1 & a_{3} \\
0 & 0 & 0 & 1
\end{array}\right) \cdot\left(\begin{array}{cccc}
1 & y & \frac{y^{2}}{2} & 0 \\
0 & 1 & y & 0 \\
0 & 0 & 1 & 0 \\
0 & 0 & 0 & 1
\end{array}\right)
$$

with given $\alpha_{i}, \beta$ and $x$ and unknown $a_{i}$ and $y$. The answer is

$$
y=x+\beta, a_{1}=\alpha_{1}+\alpha_{2} x+\frac{1}{2} \alpha_{3} x^{2}, a_{2}=\alpha_{2}+\alpha_{3} x, a_{3}=\alpha_{3} .
$$

The last step is to put in (2.2.8) $U=U_{F, A}$, where for generic representations $T_{c_{1}, c_{2}}$ with $c_{1} \neq 0$ we have to take $F=\left(c_{1}, 0, \frac{c_{2}}{2 c_{1}}, 0\right)$, while for the remaining representations $S_{c}, c \neq 0$, we put $F=(0, c, 0,0)$.

So we come to the final formulae

$$
\left(T_{c_{1}, c_{2}}\left(g\left(\alpha_{1}, \alpha_{2}, \alpha_{3}, \beta\right)\right) f\right)(x)=e^{2 \pi i\left(c_{1}\left(\alpha_{1}+\alpha_{2} x+\frac{1}{2} \alpha_{3} x^{2}\right)+\frac{c_{2}}{2 c_{1}} \alpha_{3}\right)} f(x+\beta)
$$

and

$$
\left(S_{c}\left(g\left(\alpha_{1}, \alpha_{2}, \alpha_{3}, \beta\right)\right) f\right)(x)=e^{2 \pi i c\left(\alpha_{2}+\alpha_{3} x\right)} f(x+\beta) .
$$


In conclusion we give the table of the images of basic elements of $\mathfrak{g}$ and of the central elements $A_{1}=\frac{1}{2 \pi i} X_{1}$ and $A_{2}=\frac{1}{4 \pi^{2}}\left(X_{2}^{2}-2 X_{1} X_{3}\right) \in Z(\mathfrak{g})$ under the unirreps constructed.

$\begin{array}{llll} & T_{c_{1}, c_{2}} & S_{c} & U_{a, b} \\ X_{1} & 2 \pi i c_{1} & 0 & 0 \\ X_{2} & 2 \pi i c_{1} x & 2 \pi i c & 0 \\ X_{3} & \pi i c_{1} x^{2}+2 \pi i c_{2} & 2 \pi i c x & 2 \pi i a \\ Y & \frac{d}{d x} & \frac{d}{d x} & 2 \pi i b \\ A_{1} & c_{1} & 0 & 0 \\ A_{2} & 2 c_{1} c_{2} & -c^{2} & 0\end{array}$

We observe that the commutation relations (2.2.1) are obviously satisfied and that the infinitesimal characters separate generic representations $T_{c_{1}, c_{2}}$ (but not $S_{c}$ and $S_{-c}$ ).

Example 3. Consider the subalgebra $\mathfrak{h} \subset \mathfrak{g}$ generated by $X_{1}, X_{2}$ and $Y$. It is the famous Heisenberg Lie algebra whose infinite dimensional unirreps depend on one real parameter $\hbar$ (interpreted as the Planck constant) and are given by

$$
V_{\hbar}\left(X_{1}\right)=2 \pi i \hbar, \quad V_{\hbar}\left(X_{2}\right)=2 \pi i \hbar x, \quad V_{\hbar}(Y)=\frac{d}{d x} .
$$

From the table above it is clear that $\operatorname{Res}_{H}^{G} T_{c_{1}, c_{2}}=V_{c_{1}}$. We leave it to the reader to check that it agrees with rule 3 .

Example 4. The group $G$ acts naturally by affine unimodular transformation of $\mathbb{R}^{3}$ :

$$
g\left(\alpha_{1}, \alpha_{2}, \alpha_{3}, \beta\right)(x, y, z)=\left(x+\alpha_{1}+\beta y+\frac{\beta^{2}}{2} z, y+\alpha_{2}+\beta z, z+\alpha_{3}\right) .
$$

Thus, we get a unitary representation $T$ of $G$ in $L^{2}\left(\mathbb{R}^{3}, d x d y d z\right)$. What is its spectrum (i.e. decomposition into unirreps) $?^{12}$

Observe that $T=\operatorname{Ind}_{H}^{G} 1$ where $H$ is the 1-dimensional subgroup exp $\mathbb{R} \cdot Y$ and 1 denotes the trivial 1-dimensional representation of $H$. According to rule 4 , we have to consider the projection $p_{Y}$ of $\mathfrak{g}^{*}$ on the $Y$-axis, take the $G$-saturation of the hyperplane $Y=0$ (which is $p_{Y}^{-1}(0)$ ) and decompose it into $G$-orbits. This hyperplane contains all 2-dimensional orbits and also a 1-parameter family of 0dimensional orbits. And these are exactly the unirreps which are weakly contained in $T$ in the sense of $[\mathrm{F}]$.

Since in decomposition problems one can neglect sets of measure 0 , we can restrict ourselves to generic representations. The final answer looks as follows:

$$
T=\int T_{c_{1}, c_{2}} d \mu\left(c_{1}, c_{2}\right)
$$

where $\mu$ is any measure equivalent to the Lebesgue measure on $\mathbb{R}_{c_{1}, c_{2}}^{2} \cdot{ }^{13}$

\footnotetext{
${ }^{12} \mathrm{We}$ assume here that the reader is acquainted with the decomposition theory of unitary representation; see e.g. [K2] or [K3].

${ }^{13}$ The space $L^{2}\left(\mathbb{R}^{3}, d x d y d z\right)$ has functional dimension 3 , so it is natural that it splits into a 2parameter family of spaces of functional dimension 1. This decomposition can also be interpreted as simultaneous diagonalization (spectral decomposition) of the two self adjoint Laplace operators $A_{1}$ and $A_{2}$.
} 
Example 5. Here we compute the spectrum of the tensor product $T_{c_{1}, c_{2}} \otimes S_{c}$. According to rule 5 we have to consider the arithmetic sum of $\Omega_{c_{1}, c_{2}}$, and $\Omega_{c}$. The generic points of these orbits have the coordinates $\left(c_{1}, X, \frac{c_{2}+X^{2}}{2 c_{1}}, Y\right)$ and $(0, c, x, y)$ respectively. It follows that the arithmetic sum is the hyperplane $X_{1}=c_{1}$. So, the answer is: the spectrum consists of all representations $T_{c_{1}, c_{2}}$ with fixed $c_{1}$. This agrees with the count of functional dimensions: for the tensor product the functional dimension is 2 as well as for the sum of all $T_{c_{1}, c_{2}}$ with fixed value of $c_{1}$.

Example 6. Here we shall compute the generalized character $\chi_{c_{1}, c_{2}}$ of the unirrep $T_{c_{1}, c_{2}}$.

According to rule 6 we have to compute the generalized function on $G$ given by

$$
\int_{\Omega_{c_{1}, c_{2}}} e^{2 \pi i\langle F, X\rangle+\sigma}=\int_{\Omega_{c_{1}, c_{2}}} e^{2 \pi i\langle F, X\rangle} \sigma
$$

(we use the equality $e^{\sigma}=1+\sigma$ on the 2-dimensional orbit $\Omega_{c_{1}, c_{2}}$ ).

Let's start with computation of the form $\sigma$. From (2.2.2) we get the following expression for the infinitesimal coadjoint action of $\mathfrak{g}$ on $\mathfrak{g}^{*}$ :

$$
\begin{gathered}
K_{*}\left(X_{1}\right)=0, K_{*}\left(X_{2}\right)=-X_{1} \frac{\partial}{\partial Y}, \\
K_{*}\left(X_{3}\right)=-X_{2} \frac{\partial}{\partial Y}, K_{*}(Y)=X_{1} \frac{\partial}{\partial X_{2}}+X_{2} \frac{\partial}{\partial X_{3}} .
\end{gathered}
$$

The orbit $\Omega_{c_{1}, c_{2}}$ is given by equations (2.2.3), so one can take $X:=X_{2}$ and $Y$ as global coordinates on it. In terms of these coordinates the $\mathfrak{g}$-action on $\Omega_{c_{1}, c_{2}}$ takes the form

$$
K_{*}\left(X_{1}\right)=0, K_{*}\left(X_{2}\right)=-c_{1} \frac{\partial}{\partial Y}, K_{*}\left(X_{3}\right)=-X \frac{\partial}{\partial Y}, K_{*}(Y)=c_{1} \frac{\partial}{\partial X} .
$$

In this case formula (1.2.2) implies

$$
\sigma=\frac{d X \wedge d Y}{c_{1}}
$$

We are now in a position to compute the integral above. Using the equations (2.2.3) we can rewrite it as

$$
\chi_{c_{1}, c_{2}}\left(\exp \left(\sum_{i=1}^{3} a_{i} X_{i}+b Y\right)\right)=\int_{\mathbb{R}^{2}} e^{2 \pi i\left(a_{1} c_{1}+a_{2} X+a_{3} \frac{c_{2}+X^{2}}{2 c_{1}}+b Y\right)} \frac{d X d Y}{c_{1}},
$$

and we get the final formula

$$
\chi_{c_{1}, c_{2}}\left(g\left(a_{1}, a_{2}, a_{3}, b\right)\right)=\left|a_{3} c_{1}\right|^{-\frac{1}{2}} e^{2 \pi i\left(c_{1}\left(a_{1}-\frac{a_{2}^{2}}{2 a_{3}}\right)+c_{2} \frac{a_{3}}{2 c_{1}}-\frac{1}{4} \operatorname{sgn}\left(a_{3} c_{1}\right)\right)} \delta(b) .
$$

It is worth mentioning that the same result can be obtained by formal computation of the trace of the operator (2.2.9) considered as an integral operator with distributional kernel. (For this we have to take into account that $\exp \left(\sum_{i=1}^{3} a_{i} X_{i}\right)=$ $g\left(a_{1}, a_{2}, a_{3}, 0\right)$.)

Example 7. Let us now compute the Plancherel measure $\mu$ on $\widehat{G}$. We shall use the values $c_{1}, c_{2}$ as local coordinates on $\widehat{G}$. By definition, we have

$$
\delta(g)=\int_{\widehat{G}} \chi_{c_{1}, c_{2}}(g) d \mu .
$$


The direct computation using formula (2.2.12) for the generalized character is rather complicated, but rule 10 gives the answer immediately:

$$
\mu=\frac{1}{2} d c_{1} d c_{2} .
$$

Indeed, from (2.2.3) and (2.2.9) we see that

$$
\int_{\mathfrak{g}^{*}} f\left(X_{1}, X_{2}, X_{3}, Y\right) d X_{1} d X_{2} d X_{3} d Y=\frac{1}{2} \int_{\mathbb{R}^{2}} d c_{1} d c_{2} \int_{\Omega_{c_{1}, c_{2}}} f(F) \cdot \sigma .
$$

2.3. Exponential Lie groups. Most of the prescriptions of the User's Guide above (namely, rules $1,3,4,5,8,9$ ) are still valid in this more general situation.

These results were obtained mainly by the French school; see [BCD]. The validity of rules $3,4,5$ was first proved in $[\mathrm{Bu}]$. As for the homeomorphism of $\widehat{G}$ and $\mathcal{O}(G)$ it was established only recently in [LL].

Rules 2, 6, 7 and 10 need modifications which we shall discuss below.

In rule 2 the following important correction is needed. Let us say that a subalgebra $\mathfrak{h}$ subordinate to $F \in \mathfrak{g}^{*}$ satisfies the Pukanszky condition if

$$
p^{-1}(p(F))=F+\mathfrak{h}^{\perp} \subset \Omega_{F} ;
$$

i.e. the preimage of $p(F)$ lies entirely in one $G$-orbit.

The modified rule $\mathbf{2}$ differs from the initial one by the requirement that $\mathfrak{h}$ satisfies the Pukanszky condition. In fact, the necessity of this condition follows immediately from rule 4. According to that rule the spectrum of the induced representation $\operatorname{Ind}_{H}^{G} U_{F, H}$ consists of a single point iff the Pukanszky condition is satisfied, and in this case $\operatorname{Ind}_{H}^{G} U_{F, H}$ should be a multiple of a unirrep associated with $\Omega_{F}$.

Taking into account the functional dimension argument (rule 9), we conclude that the multiplicity is 1 when the equality

$$
\operatorname{codim}_{\mathfrak{g}} \mathfrak{h}=\frac{1}{2} \operatorname{dim} \Omega_{F}
$$

holds. So, (2.3.1) and (2.3.2) together give the necessary and sufficient condition for

$$
T_{\Omega_{F}}=\operatorname{Ind}_{H}^{G} U_{F, H} .
$$

We have not included the Pukanszky condition in the initial rule 2 because of the following fact.

Proposition 1. i) Let $\mathfrak{g}$ be any Lie algebra and $\mathfrak{h} \subset \mathfrak{g}$ be any Lie subalgebra which is subordinate to $F$ and has codimension $\frac{1}{2} \operatorname{dim} \Omega$ in $\mathfrak{g}$. Then the affine manifold $F+\mathfrak{h}^{\perp}$ has open intersection with $\Omega_{F}$ (i.e. the local version of the Pukanszky condition is satisfied).

ii) If $\mathfrak{g}$ is a nilpotent Lie algebra, (2.3.1) is always satisfied.

Proof. Part i) follows from the consideration of the $H$-orbit of $F$ where $H=\exp (\mathfrak{h})$. One easily checks that this orbit is contained in $F+\mathfrak{h}^{\perp}$ and has the same dimension.

Part ii) is a consequence of $i$ ) and of the following useful fact: for nilpotent Lie groups all coadjoint orbits are affine algebraic subvarieties in $\mathfrak{g}^{*}$ (i.e. are defined by a system of polynomial equations).

For general exponential groups this is no longer true (see Example 8 below), and we have to include the Pukanszky condition in the formulation of rule 2. 
Theorem 1. (See $[\mathrm{P} 1]$ ) The modified rule 2 is valid for all exponential Lie groups.

Now we come to rule 6, which needs corrections for two reasons. First, for non-nilpotent exponential groups the generalized characters of unirreps are not necessarily well defined as distributions. Namely, it could happen that the operator $T_{\phi}$ does not belong to the trace class even for $\phi \in C_{c}^{\infty}(G)$.

The explanation of this phenomenon by the orbit method is very simple. The coadjoint orbits are no longer closed submanifolds in $\mathfrak{g}^{*}$. As a consequence, the canonical volume form associated with the symplectic structure on an orbit can have a singularity at the boundary, and the integral formula of rule 6 does not define a distribution on $G$. So, we have to restrict the domain of definition of the generalized character by imposing additional conditions on the test functions $\phi \in C_{c}^{\infty}(G)$. In the simplest case (see Example 8 below) the additional condition is very simple and natural: the Fourier transform

$$
\tilde{\phi}(F)=\int_{\mathfrak{g}} \phi(\exp X) \cdot e^{2 \pi i\langle F, X\rangle} d X
$$

should vanish on the boundary of the orbit.

Second, for nilpotent groups the Fourier transform $\phi \mapsto \tilde{\phi}$ given by (2.3.4) defines a unitary transformation from $L^{2}(G, d g)$ to $L^{2}\left(\mathfrak{g}^{*}, d F\right)$ where $d g$ is the Haar measure on $G$ and $d X$ is the corresponding Lebesgue measure on $\mathfrak{g}$. Recall that in terms of this transform rule 6 looks like

$$
\left\langle\chi_{\Omega}, \phi\right\rangle:=\operatorname{tr} T_{\Omega}(\phi)=\int_{\Omega} \tilde{\phi}(F) \cdot \frac{\sigma^{r}}{r !} \quad \text { where } \quad r=\frac{\operatorname{dim} \Omega}{2} .
$$

Or, in more simple words:

The Fourier transform of $\chi_{\Omega}$ is the canonical measure on the coadjoint orbit $\Omega$.

In the case of non-nilpotent groups one has to correct the Fourier transform (2.3.4) taking into account the more complicated relation between invariant measures on $G$ and on $\mathfrak{g}$.

For non-unimodular groups the densities of the right and left Haar measures have the following form in canonical coordinates:

$$
d_{r}(\exp X)=\operatorname{det}\left(\frac{e^{\operatorname{ad} X}-1}{\operatorname{ad} X}\right) d X, \quad d_{l}(\exp X)=\operatorname{det}\left(\frac{1-e^{-\operatorname{ad} X}}{\operatorname{ad} X}\right) d X .
$$

Let us define their "geometric mean" $q(X) d X$ by $q(X)=\operatorname{det}\left(\frac{\sinh (\operatorname{ad} X / 2)}{\operatorname{ad} X / 2}\right)$.

The function $q(X)$ is analytic on $\mathfrak{g}$, and its zeros coincide with singular points of the exponential map. For exponential groups it is everywhere positive, and so the function

$$
j(X)=\sqrt{q(X)}=\left(\operatorname{det}\left(\frac{\sinh (\operatorname{ad} X / 2)}{\operatorname{ad} X / 2}\right)\right)^{\frac{1}{2}}, j(0)=1,
$$

is well defined and analytic.

We define the modified Fourier transform by

$$
\tilde{\phi}(F)=\int_{\mathfrak{g}} e^{2 \pi i<F, X>} \phi(\exp X) j(X) d X .
$$

For unimodular exponential groups this is a unitary bijection from $L^{2}(G, d g)$ to $L^{2}\left(\mathfrak{g}^{*}, d F\right)$, and we shall use it instead of (2.3.4) in the expression (2.3.5). 
The modified rule 6 acquires the form

$$
\operatorname{tr} T_{\Omega}(\exp X)=\frac{1}{j(X)} \int_{\Omega} e^{2 \pi i\langle F, X\rangle+\sigma} .
$$

Since for nilpotent groups $j(X) \equiv 1$, the modified rule 6 coincides in this case with the original one.

It turns out that the modified rule 6 is valid for a wide class of non-nilpotent groups provided that test functions are subjected to appropriate additional restrictions.

Example 8. Let $G=\operatorname{Aff}_{+}(1, \mathbb{R})$ be the group of orientation preserving affine transformations of the real line. ${ }^{14}$ It is realized by $2 \times 2$ matrices of the form

$$
g=\left(\begin{array}{ll}
a & b \\
0 & 1
\end{array}\right), \quad a, b \in \mathbb{R}, a>0 .
$$

The elements of the Lie algebra $\mathfrak{g}$ and of the dual space $\mathfrak{g}^{*}$ are realized by real $2 \times 2$ matrices of the form

$$
X=\left(\begin{array}{cc}
\alpha & \beta \\
0 & 0
\end{array}\right), \quad F=\left(\begin{array}{cc}
x & 0 \\
y & 0
\end{array}\right)
$$

and the coadjoint action is

$$
K(a, b):(x, y) \mapsto\left(x+a^{-1} b y, a^{-1} y\right) .
$$

We see that $\mathfrak{g}^{*}$ splits into two 2-dimensional orbits $\Omega_{ \pm}=\{(x, y) \mid \pm y>0\}$ and a family of 0-dimensional orbits $\Omega_{x}=\{(x, 0)\}$.

We leave to the reader the explicit construction of unirreps for $G$ and the computation of their characters. Observe only that for any $F \in \Omega_{ \pm}$there is exactly one 1-dimensional subalgebra (the 1-dimensional ideal $\mathfrak{h} \subset \mathfrak{g}$ ) which satisfies the Pukanszky condition.

The initial formulation of rule 7 also should be corrected. The point is that the map $A \mapsto P_{A}$ of $Y(\mathfrak{g})$ to $Z(\mathfrak{g})$ defined in 2.1 via the symmetrization (2.1.1) is not an algebra homomorphism for general $\mathfrak{g}$. We shall have an opportunity to speak more about it in 6.1 and here cite only the correct formula. Namely, the modified rule 7 looks exactly as before: $\lambda_{\Omega}(A)=p_{A}(\Omega)$, but with a differently defined correspondence $A \longleftrightarrow p_{A}$.

Let $J \in \operatorname{End} S(\mathfrak{g})$ be the differential operator of infinite order with constant coefficients on $\mathfrak{g}^{*}$ which corresponds to the analytic function $j(X)$ on $\mathfrak{g}$ defined above by (2.3.6). For $A \in U(\mathfrak{g})$ we define $p_{A} \in P\left(\mathfrak{g}^{*}\right)$ so that

$$
A=\operatorname{sym}\left(J p_{A}\right) \text {. }
$$

Note that for nilpotent $\mathfrak{g}$ this definition of $p_{A}$ coincides with the initial one because $j(X) \equiv 1$ in this case.

Consider finally rule 10 concerning the Plancherel formula and the Plancherel measure. The representation-theoretic meaning of the Plancherel formula for a unimodular group $G$ is the explicit decomposition of the representation of $G \times G$ on $L^{2}(G, d g)$ into unirreps. It can be written in three equivalent ways:

\footnotetext{
${ }^{14}$ We refer to [K3], where this simple but instructive case is treated in detail (see also [BCD] and $[\mathrm{LL}])$.
} 
1. As the expression of the $\delta$-function on $G$ supported at $e$ as a (continuous) linear combination of characters:

$$
\delta(g)=\int_{\widehat{G}} \chi_{\lambda}(g) d \mu(\lambda) .
$$

2. As the inversion formula for the non-commutative Fourier transform:

$$
\phi(g)=\int_{\widehat{G}} \operatorname{tr}\left(\pi_{\lambda}(\phi) \pi_{\lambda}(g)^{*}\right) d \mu(\lambda) .
$$

3. As the analog of the classical Plancherel formula:

$$
\int_{G}|\phi(g)|^{2} d g=\int_{\widehat{G}} \operatorname{tr}\left(\pi_{\lambda}(\phi) \pi_{\lambda}(\phi)^{*}\right) d \mu(\lambda) .
$$

The measure $d \mu$ appearing in all three cases is called the Plancherel measure on $\widehat{G}$.

On general Lie groups the left and right Haar measures do not coincide. Thus, the Plancherel formula makes no sense. In terms of coadjoint orbits this is reflected by the fact that the Lebesgue measure on $\mathfrak{g}^{*}$ is no longer $K(G)$-invariant, hence can't be decomposed into canonical measures on coadjoint orbits.

But for unimodular exponential groups rule 10 is still valid (it follows from the modified rule 6).

2.4. Solvable Lie groups. There is a new phenomenon which occurs when we consider non-exponential solvable groups. Some of these groups do not belong to type I. This means that the representation theory for these groups has several unpleasant features: the topological space $\widehat{G}$ violates even the mildest separation axiom $T_{0},{ }^{15}$, the decomposition of a unitary representation into irreducible components can be essentially non-unique, there exist factor representations of types II and III in the sense of von Neumann, etc. I suggested the term wild for these groups. Accordingly, groups of type I will be called tame.

It is interesting that there are two different reasons for a solvable Lie group to be wild. Both have a simple interpretation in the orbit picture. The first reason is that the space $\mathcal{O}(G)$ can violate the semiseparation axiom. This is because coadjoint orbits are not necessarily locally closed. The simplest examples of such an occurrence were discovered by F.I. Mautner in the 50's and rediscovered later many times.

Example 9. The groups in question have dimension 5, depend on a real irrational parameter $\alpha$ and admit the matrix realization

$$
\left(\begin{array}{ccc}
e^{i t} & 0 & z \\
0 & e^{i \alpha t} & w \\
0 & 0 & 1
\end{array}\right), t \in \mathbb{R}, z, w \in \mathbb{C} .
$$

The typical coadjoint orbit is 2-dimensional with a 3-dimensional closure.

The second reason is that the canonical form $\sigma$ on some orbits can be nonexact. This innocent looking circumstance implies that after applying the standard Mackey technique (see e.g. [K2, §13]) we must consider representations of some non-abelian discrete groups which are usually wild.

\footnotetext{
${ }^{15}$ Recall that a topological space is called semiseparated or a $T_{0}$-space if for each pair of distinct points at least one possesses a neighborhood which does not contain the other.
} 
Example 10. The simplest group of this kind has dimension seven and can be realized by block-diagonal $6 \times 6$ matrices with diagonal $3 \times 3$ blocks of the form

$$
\left(\begin{array}{ccc}
e^{i s} & 0 & z \\
0 & e^{i t} & w \\
0 & 0 & 1
\end{array}\right) \text { and }\left(\begin{array}{ccc}
1 & s & r \\
0 & 1 & t \\
0 & 0 & 1
\end{array}\right), s, r, t \in \mathbb{R}, z, w \in \mathbb{C} .
$$

We refer to $[\mathrm{K} 2, \S 19]$ for the description of orbits and representations of the wild groups from examples 9 and 10.

It is natural for wild groups of the first kind to extend the notion of coadjoint orbit and consider ergodic $G$-invariant measures on $\mathfrak{g}^{*}$ as virtual coadjoint orbits. ${ }^{16}$ This was suggested in [K2] (not knowing that it had already been done in [P2], where in particular the analogue of rule 6 was obtained; the left hand side of the formula is the relative trace of the operator $T(g)$, and the right hand side is the integral over a virtual orbit).

Now we return to the tame groups. The description of the unitary dual for all solvable Lie groups of type I was obtained by L. Auslander and B. Kostant in $[\mathrm{AK}]$. We shall present here their results together with some essential complements suggested by I.M. Shchepochkina [Sh]. ${ }^{17}$

Theorem 2 (Auslander-Kostant). A connected and simply connected solvable Lie group $G$ is tame (belongs to type I) iff the space $\mathcal{O}(G)$ is semiseparated and the canonical form $\sigma$ is exact on each orbit.

Even for tame solvable groups the correspondence between coadjoint orbits and representations need not be one-to-one. To describe this correspondence we have to modify the space $\mathfrak{g}^{*}$.

The point is that, unlike the case of exponential Lie groups, a coadjoint orbit $\Omega$ need not be simply connected. Simple topological considerations show that the fundamental group $\pi_{1}(\Omega)$ is isomorphic to $\operatorname{Stab}(F) / \operatorname{Stab}^{0}(F)$ (here, as usual, $H^{0}$ denotes the connected component of the identity in $H$ ).

We define a rigged momentum as a pair $(F, \chi)$ where $F \in \mathfrak{g}^{*}$ and $\chi$ is a unitary 1-dimensional representation of $\operatorname{Stab}(F)$ such that $\chi_{*}(e)=\left.2 \pi i F\right|_{\operatorname{stab}(F)}{ }^{18}$ The set of all rigged momenta will be denoted by $\mathfrak{g}_{\text {rigg }}^{*}$. The group $G$ acts naturally on $\mathfrak{g}_{\text {rigg }}^{*}$; the corresponding orbits are called rigged orbits, and the set of all such orbits will be denoted by $\mathcal{O}_{\text {rigg }}(G)$.

Proposition 2. i) The G-action commutes with the natural projection

$$
\pi: \mathfrak{g}_{\text {rigg }}^{*} \rightarrow \mathfrak{g}^{*}:(F, \chi) \mapsto F .
$$

ii) For tame groups the projection $\pi$ is onto and the fiber over a point $F \in \mathfrak{g}^{*}$ is a torus of dimension equal to the first Betti number of $\Omega_{F}$.

So, the correspondence between usual and rigged orbits is one-to-many.

The second basic result is

\footnotetext{
${ }^{16}$ One can prove that in case of semiseparated $\mathcal{O}(G)$ all such measures are proportional to canonical measures on orbits.

${ }^{17}$ Unfortunately, the complete text of her Ph.D. thesis (Moscow, 1980) was never published and so is inaccessible for the mathematical community in the West.

${ }^{18}$ Recall that such $\chi$ exists only if the orbit $\Omega_{F}$ is integral (see Proposition 1 in 1.2). For type I solvable groups this condition is satisfied because $\sigma$ is exact.
} 
Theorem 3 (Auslander-Kostant). For a connected and simply connected solvable Lie group $G$ of type $I$ there is a natural bijection between $\widehat{G}$ and the space $\mathcal{O}_{\text {rigg }}(G)$.

We refer to the original paper $[\mathrm{AK}]$ or to $[\mathrm{K} 3]$ for the precise construction of the unirrep $T_{\Omega}$ associated with a rigged orbit $\Omega$. Here we remark only that it requires the new procedure of holomorphic induction and outline the main idea which is behind this notion. We shall speak more about it in 3.2 .

The usual induction procedure is not sufficient for the construction of a unirrep from a given rigged orbit. The reason is that for some $F \in \mathfrak{g}^{*}$ there is no subalgebra $\mathfrak{h} \subset \mathfrak{g}$ of the required dimension which is subordinate to the functional $F$. But a complex subalgebra $\mathfrak{p}$ with these properties always exists in the complexification $\mathfrak{g}_{\mathbb{C}}$.

Now, the condition (2.2.5) which we used above (see Example 2) to define the representation space $L(G, A, U)$ can be replaced by its infinitesimal version. In this form it makes sense for a complex subalgebra $\mathfrak{p}$. Namely, for $(F, \chi) \in \mathfrak{g}_{\text {rigg }}^{*}$ and for a complex subalgebra $\mathfrak{p} \in \mathfrak{g}_{\mathbb{C}}$ subordinate to $F$ we define $L(G, F, \chi, \mathfrak{p})$ as the space of complex valued functions $\phi$ on $G$ satisfying

$$
\phi(h g)=\chi(h) \phi(g) \text { for } h \in \operatorname{Stab}(F), \quad\left(L_{X}+2 \pi i\langle F, X\rangle\right) \phi=0 \text { for } X \in \mathfrak{p}
$$

where $L_{X}$ is the right invariant complex vector field on $G$ associated with $X \in \mathfrak{g}_{\mathbb{C}}$.

It turns out that under suitable conditions on $\mathfrak{p}$ (including the Pukanszky condition and the condition $\operatorname{codim}_{\mathbb{C}} \mathfrak{p}=\frac{1}{2}$ rk $B_{F}$ ) the resulting representation of $G$ by right shifts in $L(G, F, \chi, \mathfrak{p})$ is irreducible and its equivalence class depends only on the rigged orbit $\Omega$ which contains $(F, \chi)$. Therefore, we denote it by $T_{\Omega}$.

Now we discuss briefly the modified rules $3,4,5$ suggested by Shchepochkina. Let $H$ be a closed subgroup of $G$. We say that a rigged orbit $\Omega^{\prime} \in \mathcal{O}_{\text {rigg }}(H)$ lies under a rigged orbit $\Omega \in \mathcal{O}_{\text {rigg }}(G)$ (or, equivalently, $\Omega$ lies over $\Omega^{\prime}$ ) if there exist rigged momenta $(F, \chi) \in \Omega$ and $\left(F^{\prime}, \chi^{\prime}\right) \in \Omega^{\prime}$ such that the following conditions are satisfied:

$$
p(F)=F^{\prime}, \quad \chi=\chi^{\prime} \text { on } H \cap \operatorname{Stab}(F) .
$$

We also define the sum of rigged orbits $\Omega_{1}$ and $\Omega_{2}$ as the set of all $(F, \chi) \in$ $\mathcal{O}_{\text {rigg }}(G)$ for which there exist $\left(F_{i}, \chi_{i}\right) \in \Omega_{i}, i=1,2$, such that

$$
F=F_{1}+F_{2}, \quad \chi=\chi_{1} \chi_{2} \text { on } \operatorname{Stab}\left(F_{1}\right) \cap \operatorname{Stab}\left(F_{2}\right) .
$$

Theorem 4 (Shchepochkina). Let $G$ be a connected and simply connected solvable Lie group and $H$ a closed subgroup. Then

1. The spectrum of Ind ${ }_{H}^{G} S_{\Omega^{\prime}}$ consists of those $T_{\Omega}$ for which $\Omega$ lies over $\Omega^{\prime}$.

2. The spectrum of $\operatorname{Res}_{H}^{G} T_{\Omega}$ consists of those $S_{\Omega^{\prime}}$ for which $\Omega^{\prime}$ lies under $\Omega$.

3. The spectrum of $T_{\Omega_{1}} \otimes T_{\Omega_{2}}$ consists of those $T_{\Omega}$ for which $\Omega$ lies in $\Omega_{1}+\Omega_{2}$.

The proofs use the induction on the dimension of $G$ and are based on the following result, which is interesting in its own right.

Let $\mathrm{G}$ be a connected and simply connected solvable Lie group, $H$ a closed subgroup and $K$ the maximal connected normal subgroup of $G$ which is contained in $H$. Then the quotient group $G / K$ is called a basic group.

Proposition 3 (Shchepochkina). Any basic group coincides with the simply connected covering of one of the following groups:

1. The only non-commutative 2-dimensional Lie group $G_{2}=$ Aff $(1, \mathbb{R})$.

2. The one-parameter family of 3-dimensional groups $G_{3}(\gamma)$ in $A f f(1, \mathbb{C})$ acting on $\mathbb{C}$ by translations and multiplications by $e^{\gamma} t, \gamma \in \mathbb{C} \backslash \mathbb{R}$. 


\section{The 4-dimensional group $G_{4}=A f f(1, \mathbb{C})$.}

\section{The first obstacle: Compact Lie groups}

The case of compact Lie groups is the oldest and the most developed part of the representation theory of Lie groups. For classical compact groups, i.e. unitary groups over $\mathbb{R}, \mathbb{C}$, or $\mathbb{H}$, almost all basic results are known since the 20 's and were intensively used in quantum physics, which appeared just at that time. ${ }^{19}$ So, it is unlikely to expect that one can tell something new in this domain. Nevertheless, the orbit method gives new insight into the known results and even reveals some new facts.

At the same time the idyllic harmony of the unirreps-orbits correspondence starts to break down in this case. In particular, the two ways to establish this correspondence (via the induction-restriction functors from the one side and via character theory from the other one) lead to different results! ${ }^{20}$

3.1. Geometry of coadjoint orbits for compact Lie groups. Let $G$ be a compact connected Lie group. It is well known that any finite dimensional real representation of $G$ is equivalent to an orthogonal one. So, the Lie algebra $\mathfrak{g}=$ $\operatorname{Lie}(G)$ possesses a non-degenerate positive $G$-invariant bilinear form. It makes it possible to identify $\mathfrak{g}^{*}$ with $\mathfrak{g}$ and the coadjoint representation with the adjoint one.

Another well known theorem about compact transformation groups claims that for a given $G$ there are only finitely many types of coadjoint orbits as homogeneous $G$-manifolds. In other words, there are finitely many subgroups $G_{i}, 1 \leq i \leq k$, such that any orbit is isomorphic to a coset space $X_{i}=G / G_{i}$. More precisely, if $T$ is a maximal abelian subgroup in $G,{ }^{21}$ then the subgroups $G_{i}$ can be chosen so that $T \subset G_{i} \subset G$ and every group intermediate between $T$ and $G$ is conjugate to some $G_{i}$.

Example 1. Let $G=U(n), T=$ the subgroup of diagonal matrices. Then $\mathfrak{g}$ consists of all $n \times n$ skew-Hermitian matrices $X$. We can take $(X, Y):=-\operatorname{tr}(X Y)$ as an invariant bilinear form on $\mathfrak{g}$. Every (co)adjoint orbit $\Omega$ has non-empty intersection with the subspace $\mathfrak{t}=\operatorname{Lie}(T)$. Gathering equal eigenvalues of $X$ in blocks of sizes $n_{1}, \cdots, n_{r}$, we see that $\operatorname{Stab}(X)=U_{n_{1}}, \cdots, n_{r} \cong U_{n_{1}} \times \cdots \times U_{n_{r}}$. So, the number of different types of orbits is equal to the number $p(n)$ of all partitions of $n$.

The space $X=G / T$ is called the full flag manifold for $G$. The other homogeneous spaces $X_{i}=G / G_{i}$ are called degenerate flag manifolds: they can be obtained from $X$ by a projection whose fibers are isomorphic to smaller flag manifolds $G_{i} / T$.

The flag manifolds have rich geometric structure.

First, being homogeneous spaces of a compact Lie group, they admit a $G$ invariant Riemannian metric.

Second, being coadjoint orbits, they have a canonical $G$-invariant symplectic structure.

\footnotetext{
${ }^{19}$ We are not speaking about abelian compact Lie groups which were used in Fourier analysis more than two centuries ago.

${ }^{20}$ It is a temptation to consider this discrepancy as a peculiar manifestation of the Heisenberg uncertainty principle: to a given unirrep we can associate an orbit with only a certain degree of accuracy (of size $\rho=$ half sum of positive roots).

${ }^{21}$ All such subgroups are connected and belong to the same conjugacy class.
} 
Third, they can be endowed with a $G$-invariant complex structure (i.e. admit complex coordinates in which the action of $G$ is holomorphic).

Moreover, all three structures can be united in one by saying that flag manifolds are homogeneous Kähler $G$-manifolds.

We recall that a complex manifold $X$ is called Kähler if in every tangent space $T_{x}(X)$ a Hermitian form $w$ is given such that its real part $\Re w$ defines a Riemannian metric on $X$ while its imaginary part $\Im w$ defines a symplectic structure.

Locally any Kähler form $w$ can be written as $w=w_{\alpha \bar{\beta}} d z_{\alpha} d \bar{z}_{\beta}$ where the coefficients have the form $w_{\alpha \bar{\beta}}=\partial_{\alpha} \bar{\partial}_{\beta} K$ for some real-valued function $K$ which is called the Kähler potential of $w$.

Example 2. Let $G=U(n+1), X=G / U_{n, 1}=P^{n}(\mathbb{C})$. For $n \geq 2$ this is a degenerate flag manifold. Denote by $\left(x_{0}: x_{1}: \ldots: x_{n}\right)$ the homogeneous coordinates on $X$, and put

$$
K_{i}=-\log \frac{\left|x_{i}\right|^{2}}{\sum_{k=0}^{n}\left|x_{k}\right|^{2}} \text { on the affine part } X_{i} \subset X \text { where } x_{i} \neq 0 .
$$

Since the difference $K_{j}-K_{i}=\log \frac{x_{i}}{x_{j}}+\overline{\log \frac{x_{i}}{x_{j}}}$ is a sum of analytic and antianalytic functions, the corresponding Kähler form does not depend on $i$ and is well defined on $X$. In local coordinates $z_{k}=\frac{x_{k}}{x_{0}}, 1 \leq k \leq n$, the Kähler form on $X_{0}$ looks like

$$
w=\frac{\sum_{k=1}^{n}\left|d z_{k}\right|^{2}}{1+\sum_{k=0}^{n}\left|z_{k}\right|^{2}}-\frac{\sum_{k, l} \bar{z}_{k} z_{l} d z_{k} d \bar{z}_{l}}{\left(1+\sum_{k=0}^{n}\left|z_{k}\right|^{2}\right)^{2}}
$$

There is a simple explanation for why all flag manifolds possess a complex structure. To make things technically simpler we assume that $G$ is simply connected. ${ }^{22}$ Then it is a maximal compact subgroup of a complex semisimple Lie group $G_{\mathbb{C}}$ (also simply connected) with the Lie algebra $\mathfrak{g}_{\mathbb{C}}=\mathfrak{g} \otimes \mathbb{C}$. Choose a Borel (i.e. maximal connected solvable) subgroup $B \subset G_{\mathbb{C}}$ which contains $T$. Then $H=T_{\mathbb{C}}$ will be a Cartan (i.e. maximal abelian Ad-semisimple) subgroup of $G_{\mathbb{C}}$.

Consider the complex homogeneous manifold $Y=G_{\mathbb{C}} / B$. It turns out that our compact group $G$ acts transitively on it, and the stabilizer of the initial point coincides with $B \cap G=T$. So we can identify $X$ with $Y$ and obtain a $G$-invariant complex structure on the flag manifold $X$. For degenerate flag manifolds $X_{i}=G / G_{i}$ the situation is analogous; the role of $Y_{i}$ is played by $G_{\mathbb{C}} / P_{i}$ where $P_{i}$ is a so-called parabolic subgroup of $G_{\mathbb{C}}$ which contains $G_{i}$.

Remark. The $G$-invariant complex structure on a flag manifold is not unique. In the case of full flag manifold $X=G / T$ all possible complex structures form a principal homogeneous space for the Weyl group $W=N_{G}(T) / Z_{G}(T)$. This group acts by automorphisms of $X$ considered as a $G$-space and permutes the $|W|$ different $G$-invariant complex structures on $X$. (Note, that there are exactly $|W|$ different Borel subgroups containing $H$, hence $|W|$ different ways to identify $X$ with $Y$.)

From now on we fix the choice of a complex structure on $X$ or, in other words, fix a Borel subgroup $B \supset H \supset T$.

Example 3. Let $G=S U(2)$. Then $G_{\mathbb{C}}=S L(2, \mathbb{C}), X=S^{2}, Y=P^{1}(\mathbb{C})$ and the two complex structures on $S^{2}$ result from the two possible $G$-covariant identifications of $S^{2}$ with $P^{1}(\mathbb{C})$.

We leave to the reader the rather instructive

\footnotetext{
${ }^{22}$ This assumption in no way changes the coadjoint orbits.
} 
Exercise. Describe the six different invariant complex structures on $X=S U(3) / T$.

Hint. The points of the space $Y=S L(3, \mathbb{C}) / B$ are geometrically realized by pairs $V_{1} \subset V_{2}$ where $V_{i}$ is an $i$-dimensional subspace in $\mathbb{C}^{3}$. We can consider $V_{1}$ as a point of the projective plane $P^{2}(\mathbb{C})$ and $V_{2}$ (more precisely, its annihilator $V_{2}^{\perp} \subset\left(\mathbb{C}^{3}\right)^{*}$ ) as a point of a dual projective plane $P^{2}(\mathbb{C})^{*}$. We get a $G$-covariant embedding of $Y$ into the product of two dual complex projective planes. The six $G$-covariant maps $X \rightarrow Y$ correspond to the six fixpoints of the $T$-action on $Y$.

Now we discuss the topology of coadjoint orbits. Let $\Omega \subset \mathfrak{g}^{*}$ be such an orbit. The canonical symplectic form $\sigma$ on $\Omega$ defines a cohomology class $[\sigma] \in H^{2}(X, \mathbb{R})$.

Recall that an orbit $\Omega \subset \mathfrak{g}^{*}$ is called integral (see 1.2.4) if $[\sigma] \in H^{2}(X, \mathbb{Z}) \subset$ $H^{2}(X, \mathbb{R})$. The number of "integrality conditions" for a given orbit type is equal to the second Betti number of these orbits. We shall see that for compact groups the integral orbits form a discrete set.

It is well known that for a compact Lie group $G$ with $\pi_{1}(G)=0$ we also have $\pi_{2}(G)=0$. From the exact sequence

$$
\cdots \rightarrow \pi_{k}(G) \rightarrow \pi_{k}(X) \rightarrow \pi_{k-1}(T) \rightarrow \pi_{k-1}(G) \rightarrow \cdots
$$

it follows that the flag manifold $X$ is simply connected and

$$
H_{2}(X, \mathbb{Z}) \cong \pi_{2}(X) \cong \pi_{1}(T) \cong \widehat{T} \cong \mathbb{Z}^{\operatorname{dim} T} .
$$

Now let $\Omega$ be an orbit in $\mathfrak{g}^{*}$. We identify $\mathfrak{g}^{*}$ with $\mathfrak{g}$ and $\mathfrak{g}_{\mathbb{C}}^{*}$ with $\mathfrak{g}_{\mathbb{C}}$ so that $\mathfrak{h}^{*}$ goes to $\mathfrak{h}$ and the weight lattice $P \subset \mathfrak{h}^{*}$ corresponds to a lattice in $i \mathfrak{t}^{*} \subset i \mathfrak{g}^{*} \approx i \mathfrak{g}$. The intersection of $\Omega$ with $\mathfrak{t}^{*}$ is a finite set which forms a single $W$-orbit. We denote by $\Omega_{\lambda}$ the orbit passing through the point $i \lambda \in \mathfrak{t}^{*}$.

Proposition 1. i) The orbit $\Omega_{\lambda}$ is integral iff $\lambda \in P$.

ii) The dimension of $\Omega_{\lambda}$ is equal to the number of roots non-orthogonal to $\lambda$.

In particular, all orbits of maximal dimension are isomorphic as $G$-manifolds to the full flag manifold $X$.

3.2. Borel-Weil-Bott theorem. This is a culmination result in the representation theory of compact Lie groups. It gives a uniform geometric construction for all unirreps of all compact connected Lie groups.

In fact, the first part of it (the so-called Borel-Weil theorem) is the modern interpretation of the E. Cartan theory of the highest weight. It admits a beautiful generalization due to R. Bott. We want to show how these results agree with the ideology of the orbit method.

The Borel-Weil theory deals with homogeneous holomorphic line bundles $L$ on flag manifolds $X$, i.e. bundles which admit a $G$-action by holomorphic transformations. In fact, this action automatically extends to an action of the complex group $G_{\mathbb{C}}$. This allows us to describe everything in group-theoretic terms. Namely, let $\chi$ be a holomorphic character (i.e. complex one-dimensional representation) of the Borel subgroup $B$. Let $B$ act on $G_{\mathbb{C}}$ by right shifts, and denote by $\mathbb{C}_{\chi}$ the 1-dimensional complex space where $B$ acts via the character $\chi$. Then $B$ acts freely on the Cartesian product $M=G_{\mathbb{C}} \times \mathbb{C}_{\chi}$. The set of $B$-orbits in $M$ is called the fibered product over $B$ and is denoted $G_{\mathbb{C}} \times{ }_{B} \mathbb{C}_{\chi}$. It is a complex manifold which has a natural projection on $X=G_{\mathbb{C}} / B$ with a fiber $\mathbb{C}$ over each point. Hence, we can identify it with the total space of a line bundle $L_{\chi}$ over $X$. A holomorphic 
section $s: X \rightarrow L_{\chi}$ is represented by a holomorphic function $\phi_{s}$ on $G_{\mathbb{C}}$ satisfying

$$
\phi_{s}(g b)=\chi(b) \cdot \phi_{s}(g) .
$$

We denote by $\Gamma_{\text {hol }}\left(L_{\chi}\right)$ the (finite dimensional) space of all holomorphic sections of $L_{\chi}$. The group $G_{\mathbb{C}}$ acts on this space by

$$
\phi_{g \cdot s}\left(g^{\prime}\right)=\phi_{s}\left(g^{-1} g^{\prime}\right) .
$$

The representation thus obtained is called holomorphically induced from $(B, \chi)$ and is denoted by Ind $\mathrm{hol}_{B}^{G_{\mathrm{C}}} \chi$.

It is easy to describe the set of homogeneous holomorphic bundles on $X$ or, equivalently, the set of all holomorphic characters of $B$. Namely, these characters are completely determined by their restrictions to the torus $T \subset B$. These restrictions form the lattice $\widehat{T}=$ the Pontrjagin dual of $T$ which in its turn can be identified with the weight lattice $P$ in $\mathfrak{h}^{*}$ via

$$
\chi_{\lambda}(\exp X)=e^{2 \pi\langle\lambda, X\rangle}, \quad \lambda \in P, \quad X \in \mathfrak{t} .
$$

Conversely, every character (3.2.3) can be uniquely extended to a holomorphic (non-unitary) character of $B$ which is given by the same formula on $H=T_{\mathbb{C}}$ and is trivial on the commutator subgroup $N=[B, B]$.

Thus, to any $\lambda \in P$ there corresponds a character of the Borel subgroup $B$, hence a homogeneous holomorphic line bundle on $X$ which we denote by $L_{\lambda}$.

Theorem 1 (Borel-Weil). The space $\Gamma_{h o l}\left(L_{\lambda}\right)$ is non-zero exactly when $\lambda \in P_{+}$ $\left(P_{+}\right.$is the set of dominant weights) and in this case $\Gamma_{h o l}\left(L_{\lambda}\right)$ is an irreducible representation $\pi_{\lambda}$ of $G$ with highest weight $\lambda$.

The Borel-Weil theorem strongly suggests relating the representation $\pi_{\lambda}$ to the integral coadjoint orbit $\Omega_{\lambda}$ passing through $i \lambda$. In particular, the trivial representation $\pi_{0}$ should correspond to the origin in $\mathfrak{g}^{*}$. This correspondence agrees with rules 3 and 4 describing the restriction and induction functors. E.g., the set of all weights of $\pi_{\lambda}$, multiplied by $i$, coincides with $p\left(\Omega_{\lambda}\right) \cap i P$ where $p$ is the canonical projection of $\mathfrak{g}^{*}$ onto $\mathfrak{t}^{*}$. (See an example in 3.4.)

But there are even stronger arguments to associate $\pi_{\lambda}$ with the orbit $\Omega_{\lambda+\rho}$ where $\rho$ is the half sum of the positive roots of the pair $\left(\mathfrak{g}_{\mathbb{C}}, \mathfrak{h}\right)$. One of these arguments is Bott's complement to the Borel-Weil theorem. It deals with the space $H^{k}\left(X, \mathcal{L}_{\lambda}\right)$ of $k$-dimensional cohomology of $X$ with coefficients in the sheaf $\mathcal{L}_{\lambda}$ of germs of holomorphic sections of $L_{\lambda}$. For $k=0$ this reduces to the space $\Gamma_{h o l}\left(L_{\lambda}\right)$ which appears in the Borel-Weil theorem.

Theorem 2 (Bott). The space $H^{k}\left(X, \mathcal{L}_{\lambda}\right)$ is non-zero exactly when

$$
\lambda+\rho=w(\mu+\rho) \text { for some } \mu \in P_{+}, w \in W \text { and } k=l(w) \text {, the length of } w .
$$

In this case the representation of $G$ in $H^{k}\left(X, \mathcal{L}_{\lambda}\right)$ is equivalent to $\pi_{\mu}$.

This result suggests the correspondence $\pi_{\lambda} \leftrightarrow \Omega_{\lambda+\rho}$ which is a bijection between $\widehat{G}$ and the set of all integral orbits of maximal dimension. 
3.3. The integral formula for characters. Another and more visual argument in favor of the correspondence $\pi_{\lambda} \leftrightarrow \Omega_{\lambda+\rho}$ is the equality

$$
\operatorname{vol}\left(\Omega_{\lambda+\rho}\right)=\operatorname{dim} \pi_{\lambda}
$$

which is in perfect agreement with the principle of quantization: the dimension of the quantum phase space is equal to the volume of the classical phase space in Planck units. (In short, one dimension per volume unit.)

The equality (3.3.1) follows from the integral formula (2.3.5) for characters (the modified rule 6 ), which we shall discuss here in the context of compact groups. In this case the character of a unirrep is a regular (even analytic) generalized function. On the other hand, the factor $\frac{1}{j(X)}$ is defined only in the open subset $\mathcal{E} \subset \mathfrak{g}$ where the exponential map is one-to-one.

Theorem 3. (see $[\mathrm{K} 7]$ ) For $X \in \mathcal{E}$ we have

$$
\operatorname{tr} \pi_{\lambda}(\exp X)=\frac{1}{j(X)} \int_{\Omega_{\lambda+\rho}} e^{2 \pi i\langle F, X\rangle} \cdot \sigma^{r}, \quad r=\frac{\operatorname{dim} G-\operatorname{rk} G}{2} .
$$

The equality (3.2.1) is just a particular case of this theorem when $X=0$.

For the simplest case $G=S U(2)$ the theorem was stated already in [K1]. But only recently (see $[\mathrm{KV}],[\mathrm{WD}]$ ) the following remarkable property of the convolution algebra on $G$ which follows from the theorem was observed.

Consider the transform $\Phi: C^{\infty}(\mathfrak{g})^{\prime} \rightarrow C^{\infty}(G)^{\prime}$ defined by

$$
\langle\Phi(\nu), f\rangle:=\langle\nu, j \cdot(f \circ \exp )\rangle .
$$

Theorem 4. (see [WD]) For Ad G-invariant distributions the convolution operations on $G$ and $\mathfrak{g}$ (the latter considered as an abelian Lie group) are related by the transform above:

$$
\Phi(\mu) *_{G} \Phi(\nu)=\Phi\left(\mu *_{\mathfrak{g}} \nu\right) .
$$

So, the transform $\Phi$ "straightens" the group convolution, turning it into the abelian convolution on $\mathfrak{g}$. This implies in particular the following remarkable geometric fact.

Corollary. For any two (co)adjoint orbits $O_{1}, O_{2} \subset \mathfrak{g}$ the corresponding conjugacy classes $C_{1}=\exp O_{1}, C_{2}=\exp O_{2}$ possess the property

$$
C_{1} \cdot C_{2} \subset \exp \left(O_{1}+O_{2}\right) \text {. }
$$

The analytic explanation of this geometric phenomenon can be given using the property of Laplace operators, which we discuss below (see 6.1).

3.4. The multiplicity of a weight. One of the oldest (and most needed in applications) question of representation theory of compact Lie groups is the question of weight multiplicities. Given an irreducible representation $\pi_{\lambda}$ of $G$ in the space $V_{\lambda}$, one asks about the dimension $m_{\lambda}(\mu)$ of the space

$$
V_{\lambda}^{\mu}=\left\{v \in V_{\lambda} \mid H v=\langle\mu, H\rangle v \quad \forall H \in \mathfrak{h}\right\} .
$$

There are several different formulae for this quantity, but no one of them is efficient enough. The orbit method, unfortunately, is no exception. The formula suggested by this method is elegant, transparent, but (at least in its present form) not practical. 
Example 4. To show the flavor of this problem, we consider the case $G=S U(3)$. Here the weight lattice is the usual triangular lattice in $\mathbb{R}^{2}$ generated by two fundamental weights $\omega_{1}$ and $\omega_{2}$. The multiplicity function $m_{\lambda}(\mu)$ for two specific cases $\lambda=4 \omega_{1}+2 \omega_{2}$ and $\lambda=3 \omega_{1}+3 \omega_{2}$ looks like

$\begin{array}{cccccccc} & 1 & 2 & 2 & 2 & 2 & 1 \\ 1 & 2 & 3 & 3 & 3 & 2 & 1 \\ 1 & 2 & 3 & 3 & 2 & 1 \\ 1 & 2 & 3 & 2 & 1\end{array}$

$\begin{array}{cccccccc} & 1 & 1 & 1 & 1 & \\ 1 & 2 & 2 & 2 & 1 & \\ 1 & 2 & 3 & 3 & 2 & 1 \\ 1 & 2 & 3 & 4 & 3 & 2 & 1 \\ 1 & 2 & 3 & 3 & 2 & 1 \\ 1 & 2 & 2 & 2 & 1 \\ 1 & 1 & 1 & 1\end{array}$

One can easily guess from these pictures the general recipe which is valid for any unirrep of $S U(3)$. Namely, the support of $m_{\lambda}(\mu)$ is a convex hexagon whose vertices form a $W$-orbit of the highest weight $\lambda$. The values of $m_{\lambda}(\mu)$ along the perimeter of the hexagon are equal to 1 , on the next layer they are equal to 2 and continue to grow linearly until the hexagonal layer degenerates to a regular triangle. Then the growth stops (on the value $\min (k, l)+1$ for $\lambda=k \omega_{1}+l \omega_{2}$ ).

For general compact groups it is also known that the support of $m_{\lambda}(\mu)$ coincides with the convex hull $\Pi_{\lambda}$ of the $W$-orbit of $\lambda$ and that $m_{\lambda}(\mu)$ is given by a piecewise polynomial function. Moreover, the domains where the multiplicity is given by a certain polynomial are known. They are bounded by hyperplanes

$$
H_{w, \alpha}=w(\lambda)+\alpha^{\perp}, w \in W, \alpha \in \Delta_{+} .
$$

On the other hand, it is known that the image of $\Omega_{\lambda}$ under the canonical projection $p: \mathfrak{g}^{*} \rightarrow \mathfrak{t}^{*}$ coincides with the polytope $i \Pi_{\lambda}$. Moreover, if we denote by $S$ the subset of those points of $\Omega_{\lambda}$ where the projection is not a submersion, then the image of $S$ under $p$ is the union of intersections $i H_{w, \alpha} \cap p\left(\Omega_{\lambda}\right)$. In other words, the multiplicity formula changes its form exactly where the preimage $p^{-1}(x)$ can change.

This suggests that the weight multiplicity $m_{\lambda}(\mu)$ is related to the geometry of the preimage $p^{-1}(i \mu) \cap \Omega_{\lambda}$. It is indeed so, but only under the condition that we replace $\Omega_{\lambda}$ by $\Omega_{\lambda+\rho}$ !

Namely, let $X_{\lambda+\rho}^{\mu}$ be the quotient of $p^{-1}(i \mu) \cap \Omega_{\lambda+\rho}$ by the action of the centralizer of $\mu$ in $G$. This is the so-called reduced symplectic manifold (see [AG]). It turns out that the most naive conjecture

$$
m_{\lambda}(\mu)=\operatorname{vol} X_{\lambda+\rho}^{\mu}
$$

is "asymptotically true" (see $[\mathrm{H}])$.

There is a vast literature around the more sophisticated forms of this conjecture involving the Todd genus and the Riemann-Roch number of the manifold $X_{\lambda+\rho}^{\mu}$. We refer to [GS], [GLS] and to the papers quoted there. My personal impression is that the right formula for multiplicities is still to be found.

3.5. Application to the symmetrization map. The integral formula (3.3.2) and equality (3.3.4) together imply the modified rule 7 , and the latter can be used to get the explicit form of the isomorphism sym $: Y(\mathfrak{g}) \rightarrow Z(\mathfrak{g})$. We show it on the simplest 
Example 5. Let $G=S U(2), \mathfrak{g}=\mathfrak{s u}(2)$ with the standard basis $X, Y, Z$ and the commutation relations

$$
[X, Y]=Z, \quad[Y, Z]=X, \quad[Z, X]=Y .
$$

We denote by small letters $x, y, z$ the same elements considered as coordinates on $\mathfrak{g}^{*}$ and by $\alpha, \beta, \gamma$ the dual coordinates on $\mathfrak{g}$. Put also

$$
r=\sqrt{x^{2}+y^{2}+z^{2}}, \quad \rho=\sqrt{\alpha^{2}+\beta^{2}+\gamma^{2}} .
$$

It is clear that in our case $Y(\mathfrak{g})=\mathbb{C}\left[r^{2}\right]$ and $Z(\mathfrak{g})=\mathbb{C}[C]$ where

$$
C:=X^{2}+Y^{2}+Z^{2}=\operatorname{sym}\left(r^{2}\right) \in Z(\mathfrak{g}) .
$$

Unfortunately, the map sym $: \mathbb{C}[x, y, z] \rightarrow U(\mathfrak{g})$ is not easily computable even when restricted to $Y(\mathfrak{g})=\mathbb{C}\left[r^{2}\right]$. E.g. one can check that $\operatorname{sym}\left(r^{4}\right)=C^{2}+\frac{1}{3} C{ }^{23}$ but the direct computation of $\operatorname{sym}\left(r^{6}\right)$ is already rather complicated. So we choose the roundabout way based on the modified rule 7 .

It is instructive to compare this problem with the computation of the various symbols of differential operators (cf. [K2, $\S 18$, no. 2]).

The function $j$ on $\mathfrak{g}$ in our case takes the form

$j(\alpha, \beta, \gamma)=\left(\operatorname{det}\left(\frac{\sinh (\operatorname{ad}(\alpha X+\beta Y+\gamma Z) / 2)}{\operatorname{ad}(\alpha X+\beta Y+\gamma Z) / 2}\right)\right)^{\frac{1}{2}}=\frac{\sin (\rho / 2)}{\rho / 2}=1-\frac{\rho^{2}}{24}+\cdots$.

Recall that we identify polynomials on $\mathfrak{g}$ with differential operators with constant coefficients on $\mathfrak{g}^{*}$, so that $\rho^{2}$ goes to the operator $\Delta=\partial_{x}^{2}+\partial_{y}^{2}+\partial_{z}^{2}$ and the function $j$ corresponds to some differential operator of infinite order $J$.

Since the restriction of the operator $\Delta$ to $Y(\mathfrak{g})$ is given by the simple expression $\Delta=r^{-1} \circ \frac{d^{2}}{d r^{2}} \circ r$, we get

$$
(J F)(r)=r^{-1} \frac{\sin \left(\frac{1}{2} \frac{d}{d r}\right)}{\frac{1}{2} \frac{d}{d r}}(r F(r))=F(r)-\frac{(r F(r))^{\prime \prime}}{24 r}+\ldots
$$

and, in particular,

$$
J r^{2}=r^{2}-\frac{1}{4}, \quad J \frac{\sinh a r}{a r}=\frac{\sin (a / 2)}{a / 2} \cdot \frac{\sinh a r}{a r} .
$$

The modified rule 7 implies that the map symo $J$ restricted on $Y(\mathfrak{g})$ is an algebra homomorphism; hence for any power series $f$ we have

$$
\operatorname{sym}\left((J f)\left(r^{2}\right)\right)=f\left(\operatorname{sym}\left(J r^{2}\right)\right)=f\left(C-\frac{1}{4}\right) .
$$

So, from (3.5.3) we obtain

$$
\operatorname{sym}\left(\frac{\sinh a r}{a r}\right)=\frac{a / 2}{\sin (a / 2)} \cdot \frac{\sin \left(a \sqrt{\frac{1}{4}-C}\right)}{a \sqrt{\frac{1}{4}-C}} .
$$

\footnotetext{
${ }^{23}$ In the lectures by Roger Godement on Lie groups this computation is accompanied by the remark: "Resultat qui n'incite pas à pousser plus loin les investigations, encore que les physiciens procedent tout les jours depuis le debut des années 30 à des calculs de ce genre."
} 
This gives the following explicit expression for the map sym $: Y(\mathfrak{g}) \rightarrow Z(\mathfrak{g})$ :

$$
\operatorname{sym}\left(r^{2 n}\right)=\frac{(-1)^{n-1}}{4^{n}} \sum_{k=0}^{n}\left(\begin{array}{c}
2 n+1 \\
2 k
\end{array}\right) B_{2 k}\left(4^{k}-2\right)(1-4 C)^{n-k}
$$

where $B_{2 k}$ are Bernoulli numbers.

3.6. Intertwining operators. Another question important in applications is the structure of intertwining, or $G$-covariant operators between two representation spaces. Many remarkable differential and integral operators can be interpreted (or even defined) as intertwining operators. E.g. the Laplace operator

$$
\Delta=\sum_{i=1}^{n}\left(\frac{\partial}{\partial x_{i}}\right)^{2}
$$

is an intertwining operator for the natural action of the Euclidean motion group in any function space on $\mathbb{R}^{n}$. Other examples are the Fourier and Radon transforms.

The big deficiency of the orbit method is that until now it helped very little in the study of intertwining operators. (Though there is a beautiful formula for the so called intertwining number of two representations in terms of the symplectic geometry - see [GS].)

The reason is that a natural correspondence exists between orbits and equivalence classes of unirreps rather than unirreps themselves. The construction of an individual representation $T$ from a given equivalence class defined by an orbit $\Omega$ needs some arbitrary choice (e.g. the choice of a representative $F \in \Omega$ and a subalgebra $\mathfrak{h}$ subordinate to $F$ ).

Recently a new approach to the representation theoretic study of special functions was suggested in [EKJ] and developed in [EFK]. It requires a detailed description of intertwining operators for certain geometric representations of compact groups. I consider as a very challenging problem the application of the orbit method to these questions.

\section{More troubles: Non COMPaCt SEmisimple Groups}

The systematic study of unirreps for non compact semisimple groups was started independently by Gelfand and his school and by Harish-Chandra at the end of the 40 's, soon after the pioneering paper by Bargmann $[\mathrm{Ba}]$ on $S L(2, \mathbb{R})$ and GelfandNaimark $[\mathrm{G}]$ on $S L(2, \mathbb{C})$.

They discovered that the set $\widehat{G}$ of unirreps of a given semisimple group $G$ splits into different series. A posteriori these series can be related to different types of (co)adjoint orbits, though the correspondence here is not so simple as for the case of compact groups.

4.1. Principal and degenerate series. The principal series of representations were defined first for complex semisimple groups and for those real semisimple groups $G$ which admit the so-called split Cartan subalgebra $\mathfrak{h} \subset \mathfrak{g}$ (such that $\mathfrak{h}_{\mathbb{C}}$ is a Cartan subalgebra in $\mathfrak{g}_{\mathbb{C}}$ and the roots of $\left(\mathfrak{g}_{\mathbb{C}}, \mathfrak{h}_{\mathbb{C}}\right)$ take real values on $\mathfrak{h})$. The series consists of representations which are induced from 1-dimensional representations of the Borel subgroup $B \supset H=\exp \mathfrak{h}$. So, in the real case they form a continuous family depending on $l=\operatorname{rk} G$ parameters and have the functional dimension $r=\#\left(\Delta_{+}\right)=\frac{\operatorname{dim} G-\operatorname{rk} G}{2}$. 
The degenerate series arises when one replaces $B$ by a parabolic subgroup $P \supset B$. They have a functional dimension less than $r$ and form a family depending on less than $l$ parameters.

All these series are in a perfect correspondence with the family of (co)adjoint orbits which have a non-empty intersection with $\mathfrak{h}$. Moreover, for the principal series the modified rule 6 (character formula) is valid in the domain $\mathcal{E} \subset \mathfrak{g}$ where the exponential map is a diffeomorphism. (See [Du1] where a more general fact is proved.)

But there is no way to extend this formula to the degenerate series (even for the simplest non-trivial case $g=S L(3, \mathbb{R})$ ).

Example 1. Let $G=S L(2, \mathbb{R})$. The space $\mathfrak{g}^{*}$ consists of matrices

$$
f=\left(\begin{array}{cc}
x & y+z \\
y-z & -x
\end{array}\right)=x X+y Y+z Z
$$

The center $Z(\mathfrak{g})$ of $U(\mathfrak{g})$ is generated by the element $C=X^{2}+Y^{2}-Z^{2}$. So, the coadjoint orbits are one-sheeted hyperboloids $\Omega_{r}: x^{2}+y^{2}-z^{2}=r^{2}>0$, upper and lower sheets of the two-sheeted hyperboloids $\Omega_{ \pm i r}: x^{2}+y^{2}-z^{2}=-r^{2}<0, \pm z>0$, two sheets $\Omega_{ \pm}$of the cone $x^{2}+y^{2}=z^{2}, \pm z>0$ and the origin $\Omega_{0}$.

The principal series correspond to the family of one-sheeted hyperboloids $\Omega_{r}$. It consists of representations $T_{r}$ induced from a 1-dimensional representation of the upper triangular subgroup $B$. Repeating the procedure described in Example 2 of 2.2 , we get the explicit formula:

$$
\left[T_{r}\left(\begin{array}{ll}
a & b \\
c & d
\end{array}\right) f\right](t)=f\left(\frac{a t+c}{b t+d}\right) \cdot|b t+d|^{-1+2 \pi i r} .
$$

Remark. In fact, the orbits $\Omega_{r}$ are not simply connected. So to every such orbit there corresponds a bunch of unirreps labelled by points of a circle group (dual to $\left.\pi_{1}\left(\Omega_{r}\right) \cong \mathbb{Z}\right)$. However, they are well defined only for the universal cover of $G$. For $G$ itself only two representations are single-valued: the one given by (4.1.2) and another one with additional factor $\operatorname{sign}(b t+d)$. (And for the group $P S L(2, \mathbb{R}$ ) there remains only the representation (4.1.2).)

4.2. Discrete series. By definition, a unirrep $\pi$ belongs to the discrete series if it enters as a direct summand in the regular representation of $G$ which acts in $L^{2}(G, d g)$ by left shifts.

The well-known result of Harish-Chandra states that a real semisimple Lie group $G$ possesses a discrete series of unirreps iff it has a compact Cartan subgroup.

This fact can be easily explained (but not proved) by the orbit method. Indeed, the generic coadjoint orbits split into families which are naturally labelled by the conjugacy classes of Cartan subgroups. For a fixed Cartan subgroup $H$ the corresponding family consists of orbits which intersect $\mathfrak{h}=\operatorname{Lie}(H)$ in a regular point $X$. All such orbits are isomorphic as $G$-manifolds to the space $G / H$. The same topological argument as we used in 3.1 shows that

$$
b_{2}(G / H):=\operatorname{dim} H^{2}(G / H, \mathbb{R})=\operatorname{dim} H_{c}
$$

where $H_{c}$ is a maximal compact subgroup in $H$.

So, the number of the integrality conditions is equal to $\operatorname{dim} H_{c}$, while the total number of parameters of an orbit from the given family is equal to $\operatorname{dim} H$. It follows that integral orbits form a discrete set iff $\operatorname{dim} H=\operatorname{dim} H_{c}$, i.e. when $H=H_{c}$. 
Example 2. Historically the first example of discrete series was constructed in [Ba] for $G=S L(2, \mathbb{R})$. These representations $T_{ \pm i r}$ correspond to the orbits $\Omega_{ \pm i r}$ and in fact form a continuous family, but only those for which $r \in \mathbb{Z} \backslash\{0\}$ are single-valued on $G$. They are holomorphically induced from a 1-dimensional representation of the maximal compact subgroup $K \subset G$ and act by the formula

$$
\left[T_{n}\left(\begin{array}{ll}
a & b \\
c & d
\end{array}\right) f\right](z)=f\left(\frac{a z+c}{b z+d}\right) \cdot(b z+d)^{-|n|}, \quad n \in \mathbb{Z} \backslash\{0\}
$$

in the space of holomorphic functions on the upper or lower half plane $H_{ \pm} \cong G / K$ endowed with an appropriate inner product.

The important role of the series introduced above is that any unirrep which is weakly contained ${ }^{24}$ in the regular representation $R$ of $G$ can be constructed as some combination of them. Namely, let $P$ be some parabolic subgroup with a semisimple Levi subgroup $S$ and a unipotent radical $N$. Suppose that $S$ has a compact Cartan subgroup and consequently possesses a discrete series of unirreps. Let $\pi$ be one of these unirreps, and denote by $\tilde{\pi}$ the corresponding representation of $P$ (which coincides with $\pi$ on $S$ and is trivial on $N$ ). Finally, put $T=\operatorname{Ind}_{P}^{G} \tilde{\pi}$.

Theorem 1 (Harish-Chandra). The procedure described above yields enough unirreps to decompose $L^{2}(G, d g)$ and prove the Plancherel formula.

Theorem 2 (Rossmann). For a reductive real Lie group the modified rule 6 holds for the characters which occur in the Plancherel formula.

4.3. Complementary series. There is also a very interesting complementary series of unirreps which are not weakly contained in $R$. They can be obtained from the principal and degenerate series by analytic continuation. We shall speak more about this phenomenon in 8.4.

Example 3. We use the same group $G=S L(2, \mathbb{R})$ and define a representation by the formula

$$
\left[T_{s}\left(\begin{array}{ll}
a & b \\
c & d
\end{array}\right) f\right](t)=f\left(\frac{a t+c}{b t+d}\right) \cdot|b t+d|^{-1-s}, \quad 0<s<1
$$

in the space of functions on $P^{1}(\mathbb{R})$ with non-local inner product

$$
\left(f_{1}, f_{2}\right)=\int_{-\infty}^{\infty} \int_{-\infty}^{\infty} f_{1}\left(t_{1}\right) \overline{f_{2}\left(t_{2}\right)} \cdot\left|t_{1}-t_{2}\right|^{s-1} d t_{1} d t_{2} .
$$

4.4. Metaplectic representation and its analogs. Every element $X$ of a real semisimple Lie algebra $\mathfrak{g}$ can be uniquely written in the form

$$
X=X_{s}+X_{n}, \quad\left[X_{s}, X_{n}\right]=0
$$

where $X_{s}$ is ad-semisimple and $X_{n}$ is ad-nilpotent (that is, the operator ad $X_{s}$ can be diagonalized and $\left(\operatorname{ad} X_{n}\right)^{k}=0$ for some $k$ ). Accordingly, the general (co)adjoint orbit $\Omega_{X} \subset \mathfrak{g}^{*}$ can be fibered over the semisimple orbit $\Omega_{X_{s}} \subset \mathfrak{g}^{*}$ with the fiber a nilpotent orbit $\omega_{X_{n}} \subset \operatorname{stab}\left(X_{s}\right)^{*}$.

The unirreps of the principal, discrete and degenerate series are related to semisimple orbits, while the nilpotent orbits correspond to some (if any) exceptional representations which are called unipotent. In particular, an important example of a nilpotent orbit arises when $\mathfrak{g}$ is a simple complex Lie algebra, $\psi$ is

\footnotetext{
${ }^{24}$ In the sense of Fell [F]; see also [K2].
} 
a maximal root and we consider the orbit $\Omega_{\psi}$ which goes through a root vector $E_{\psi} \in \mathfrak{g} ; \Omega_{\psi}$ is called the minimal coadjoint orbit for $\mathfrak{g}$. In the real situation, when $\mathfrak{g}$ is a split real simple algebra, there could be several minimal coadjoint orbits.

We consider here the most interesting case when $\mathfrak{g}=\mathfrak{s p}(2 n, \mathbb{R})$ is the real symplectic Lie algebra. It consists of matrices $X$ satisfying

$$
X^{t} J+J X=0
$$

where $J=-J^{t}$ is a skew-symmetric non-degenerate matrix (the matrix of the symplectic form on $\mathbb{R}^{2 n}$ ). This Lie algebra as a vector space and as a $\mathfrak{g}$-module can be identified with the space $S y m^{2}\left(\mathbb{R}^{2 n}\right)$ of all real symmetric matrices of order $2 n$ via the map

$$
\mathfrak{s p}(2 n, \mathbb{R}) \ni X \mapsto J X \in \operatorname{Sym}^{2}\left(\mathbb{R}^{2 n}\right) .
$$

Among the non-zero $S p(2 n, \mathbb{R})$-orbits in $S y m^{2}\left(\mathbb{R}^{2 n}\right)$ there are two orbits $\Omega_{ \pm}$of the minimal possible dimension $2 n$; they consist of symmetric matrices of rank 1 which are non-positive or non-negative. These orbits are exactly the minimal coadjoint orbits for $\mathfrak{g}$.

The usual methods of constructing a unirrep from an orbit do not work in this case: there is no appropriate subalgebra either in $\mathfrak{g}$ or in its complexification. But we can use an indirect way to show that these orbits correspond to the famous Segal-Shale-Weil (or oscillator) representation of $S p(2 n, \mathbb{R}$ ) (see e.g. [K5]). In fact, it is a projective representation which is a genuine linear representation of the double cover of $S p(2 n, \mathbb{R})$, the so-called metaplectic group $M p(2 n, \mathbb{R})$.

Consider the so-called triangular symplectic group $T \operatorname{Sp}(2 n+2, \mathbb{R})$ which is by definition the stabilizer of a point for the natural action of $S p(2 n+2, \mathbb{R})$ on $\mathbb{R}^{2 n+2} \cdot{ }^{25}$ We realize the Lie algebra $\mathfrak{s p}(2 n+2, \mathbb{R})$ as the space of matrices $A$ satisfying

$$
A^{t} \cdot\left(\begin{array}{ccc}
0 & 0 & 1 \\
0 & J & 0 \\
-1 & 0 & 0
\end{array}\right)+\left(\begin{array}{ccc}
0 & 0 & 1 \\
0 & J & 0 \\
-1 & 0 & 0
\end{array}\right) \cdot A=0
$$

which implies

$$
A=\left(\begin{array}{ccc}
a & y^{t} & z \\
J q & X & J y \\
r & -q^{t} & -a
\end{array}\right) \quad \text { where } \quad X^{t} J+J X=0, \quad \text { i.e. } X \in \mathfrak{s p}(2 n, \mathbb{R})
$$

The Lie subalgebra $\operatorname{tap}(2 n+2, \mathbb{R})=\operatorname{stab}(0,0,1)$ is defined by the conditions $a=0, q=0, r=0$. Hence, it is a semidirect product of a subalgebra $\mathfrak{s p}(2 n, \mathbb{R})$ and a nilpotent normal subalgebra $\mathfrak{h}_{n}$ of dimension $2 n+1$ - the so-called Heisenberg algebra. Correspondingly, $T S p(2 n+2, \mathbb{R})$ is a semidirect product of a subgroup $S p(2 n, \mathbb{R})$ and a nilpotent normal subgroup $H_{n}$.

Let us identify as usual $\mathfrak{s p}(2 n+2, \mathbb{R})$ with its dual. Then $\mathfrak{t} \mathfrak{s p}(2 n+2, \mathbb{R})^{*}$ will consist of matrices

$$
F=\left(\begin{array}{ccc}
0 & 0 & 0 \\
J q & X & 0 \\
r & -q^{t} & 0
\end{array}\right) \quad \text { where } \quad X \in \mathfrak{s p}(2 n, \mathbb{R})
$$

\footnotetext{
${ }^{25}$ This is one of several candidates for the role of the "odd symplectic group" $S p(2 n+1, \mathbb{R})$.
} 
There is a family of coadjoint orbits $\Omega_{h}$ which have the minimal dimension $2 n$, depend on a real non-zero parameter $h$ and are defined by the equations:

$$
r=h, \quad J X=q h^{-1} q^{t} .
$$

They possess the following geometric properties.

1. Under the natural projection on $\mathfrak{h}_{n}^{*}$ the orbit $\Omega_{h}$ goes to the orbit which corresponds to the unirrep $V_{h}$ of the Heisenberg group $H_{n}$ (cf. Example 3 in 2.2 where the case $n=1$ is discussed).

2. Under the projection on $\mathfrak{s p}(2 n, \mathbb{R})^{*}$ the orbit $\Omega_{h}$ goes to $\Omega_{\text {sign }(h)}$.

According to rule 3 of the User's Guide this means that there should exist a unirrep $\pi_{h}, h \in \mathbb{R} \backslash\{0\}$, of $T S p(2 n+2, \mathbb{R})$ (or its universal cover) with the properties:

$$
\operatorname{Res}_{H_{n}}^{T S p(2 n+2, \mathbb{R})} \pi_{h} \approx V_{h}, \quad \operatorname{Res}_{S p(2 n+2, \mathbb{R})}^{T S p(2 n+2, \mathbb{R})} \pi_{h} \approx \pi_{\text {sign }(h)}
$$

where $\pi_{ \pm}$are some unirreps of $S p(2 n, \mathbb{R}$ ) (or its universal cover) corresponding to the orbits $\Omega_{ \pm} \in \mathfrak{s p}(2 n, \mathbb{R})^{*}$.

The reality is, however, slightly different from this picture. Namely, the representation space of $V_{h}$ admits a unique unitary action $\pi_{h}$ of the so-called metaplectic group $M p(2 n, \mathbb{R})$, a double cover of $S p(2 n, \mathbb{R})$, so that we get a representation of the semidirect product $M p(2 n, \mathbb{R}) \ltimes H_{n}$. The equivalence class of $\pi_{h}$ does indeed depend only on $\operatorname{sign}(h)$, but the resulting equivalence classes $\pi_{ \pm}$are reducible! They split into two irreducible components.

Example 4. Let $n=1$. The Lie algebra $\operatorname{tap}(4, \mathbb{R})$ consists of matrices

$$
M=\left(\begin{array}{cccc}
0 & -y & x & z \\
0 & a & b & x \\
0 & c & -a & y \\
0 & 0 & 0 & 0
\end{array}\right)=a A+b B+c C+x X+y Y+z Z
$$

where the basic elements satisfy

$$
\begin{gathered}
{[X, Y]=2 Z, \quad[A, B]=2 B, \quad[A, C]=-2 C, \quad[B, C]=A,} \\
{[A, X]=X, \quad[A . Y]=-Y, \quad[B, Y]=X, \quad[C, X]=Y,}
\end{gathered}
$$

the other commutator being zero.

Recall that the representation $V_{h}$ sends the generators of $\mathfrak{h}_{1}$ to the operators

$$
V_{h}(X)=\frac{d}{d t}, \quad V_{h}(Y)=2 i h t, \quad V_{h}(Z)=i h .
$$

From the commutation relations (4.4.6) we derive that

$$
\pi_{h}(A)=-t \frac{d}{d t}-\frac{1}{2}, \quad \pi_{h}(B)=\frac{1}{4 i h} \frac{d^{2}}{d t^{2}}, \quad \pi_{h}(C)=-i h t^{2} .
$$

In particular, we get

$$
\pi_{h}(\exp \theta(B-C)) \phi_{0}=e^{i \frac{\theta}{2}} \phi_{0}, \quad \phi_{0}(t)=e^{-|h| t^{2}}
$$

which shows that $\pi_{h}$ is well defined only on the double cover of $S p(2, \mathbb{R})$.

We see also that the equivalence class of $\pi_{h}$ depends only on the sign of $h$ and that subspaces of even and odd functions are invariant under all operators (4.4.7). More detailed study shows that these subspaces are irreducible, so we get four oscillator unirreps.

The oscillator representations play quite a remarkable role in all of representation theory. Here we note only two of their features: 
1. They form the base of the so-called Howe duality (see [Ho2]) - a new branch of representation theory where the orbit method has not yet been used to the fullest.

2. They enter in the construction of a unirrep from an orbit suggested in [Du3] which is based on the embedding of $\operatorname{StabF}$ in $S p\left(T_{F} \Omega, \sigma\right)$ and leads to a notion of admissible data which is a modification of the rigged momenta introduced in 2.4.

The thorough study of minimal orbits and associated representations is now in progress (see $[\mathrm{BK}],[\mathrm{V}]$ ), but it is too early to describe it here.

\section{BEYOND THE LIE GROUPS}

One of the merits of the orbit method is that it could be applied not only for the Lie groups in the classical sense. Indeed, the key notion of coadjoint orbit makes sense whenever the cotangent space is defined. In particular, this is the case if the group $G$ in question is an algebraic group over any field $K$. Another possibility is to consider groups whose underlying sets have the structure of an infinite dimensional manifold. And, finally, one can consider quantum groups which are not groups at all but still have analogs of tangent and cotangent spaces (which are dual Poisson-Lie groups). In this chapter we discuss these aspects of the orbit method.

5.1. $\mathfrak{p}$-adic and adelic groups. The invention of $\mathfrak{p}$-adic fields and rings of adeles brought into number theory powerful new methods, including global, functional and harmonic analysis. This caused tremendous progress, resulting recently in the proof of the Taniyama-Weil conjecture and, as a corollary, Fermat's Last Theorem. ${ }^{26}$

This is not the place to explain these notions in full detail. Recall only that by a global field $K$ one usually understands either an algebraic number field which is a finite extension of $\mathbb{Q}$ or a function field which is a finite extension of $\mathbb{F}_{q}(t)$ where $\mathbb{F}_{q}$ is a finite field with $q$ elements.

A local field $K_{\mathfrak{p}}$ is the completion of a global field $K$ with respect to some norm $\|\cdot\|_{\mathfrak{p}}$ satisfying

$$
\|x+y\|_{\mathfrak{p}} \leq\|x\|_{\mathfrak{p}}+\|y\|_{\mathfrak{p}}, \quad\|x y\|_{\mathfrak{p}}=\|x\|\left\|_{\mathfrak{p}}\right\| y \|_{\mathfrak{p}} .
$$

It could be $\mathbb{R}, \mathbb{C}$ or some non-archimedian field in which the triangle inequality from (5.1.1) is replaced by the ultrametric inequality

$$
\|x+y\|_{\mathfrak{p}} \leq \min \left(\|x\|_{\mathfrak{p}},\|y\|_{\mathfrak{p}}\right) .
$$

The adelic ring $\mathbb{A}(K)$ is the restricted topological product of all local fields associated with $K$. The characteristic property of this topological ring is the selfduality (in the Pontriagin sense) of the exact sequence:

$$
0 \rightarrow K \rightarrow \mathbb{A}(K) \rightarrow \mathbb{A}(K) / K \rightarrow 0
$$

where $K$ is considered as a discrete topological group. ${ }^{27}$

In my talk at the Moscow ICM (1966) it was shown that the ideology of the orbit method still works for some $\mathfrak{p}$-adic and adelic Lie groups. For groups of this type some theorems can be proved mutatis mutandum. E.g., the theory of

\footnotetext{
${ }^{26}$ It is remarkable that the Taniyama-Weil conjecture can be formulated in representationtheoretic language (in terms of two-dimensional p-adic representations of the Galois group $\operatorname{Gal}(\overline{\mathbb{Q}} / \mathbb{Q})$ related to elliptic curves), but this question is beyond the usual scope of representation theory.

${ }^{27}$ The well known example of a self-dual sequence is $0 \rightarrow \mathbb{Z} \rightarrow \mathbb{R} \rightarrow \mathbb{R} / \mathbb{Z} \rightarrow 0$. It is helpful to keep in mind the analogy between the two sequences.
} 
unitary representations of nilpotent groups over a local field of characteristic zero is completely parallel to the real case. For groups over the adele rings $\mathbb{A}(K)$ where $K$ is an algebraic number field, the situation is more delicate: these groups are usually "wild" (i.e. do not belong to type I in the sense of von Neumann). But still the ideology of the orbit method works nicely and gives a simple description of most important unirreps. The point is that the additive groups of $\mathbb{Q}_{\mathfrak{p}}$ and $\mathbb{A}(K)$ are self dual in the Pontriagin sense. So, one has natural candidates for the space $\mathfrak{g}^{*}$ and the coadjoint orbits.

Some propositions suggested by the orbit method remain true in $\mathfrak{p}$-adic or adelic situations but require different proofs. The most brilliant example is the decomposition of the space $L^{2}\left(G_{\mathbb{A}(\mathbb{Q})} / G_{\mathbb{Q}}\right)$ for "nice" algebraic groups $G$. In the case of semisimple groups it is the main object of the Langlands program.

The ideology of the orbit method suggests that the spectrum of $L^{2}\left(G_{\mathbb{A}(\mathbb{Q})} / G_{\mathbb{Q}}\right)$ should consist of unirreps corresponding to those orbits in $\mathfrak{g}^{*}(\mathbb{A}(\mathbb{Q}))$ which have nonempty intersection with $\mathfrak{g}(\mathbb{Q})^{\perp}=\mathfrak{g}^{*}(\mathbb{Q}) \subset \mathfrak{g}^{*}(\mathbb{A}(\mathbb{Q})$ ). And in the case of nilpotent groups this is indeed the case, as is shown in $[\mathrm{M}]$, [Ho1]. Even the traditionally hard question about multiplicities in this case can be almost solved in terms of orbits.

As another example of the heuristic power of the orbit method (which is not however reinforced by a rigorously proved theorem) I want to quote one question I was recently asked by I. Piatetsky-Shapiro. Let $K$ be a non-discrete locally compact field of characteristic 0 . Then $K$ is self-dual. More precisely, if we fix a non-trivial additive character $\chi$, then the general character has the form $\chi_{a}(x):=\chi(a x)$.

Consider the group $G=G L(2 n, K)$ and the subgroup $H$ of matrices of the form:

$$
\left(\begin{array}{cc}
A & B \\
0 & A
\end{array}\right), \quad \text { where } A \in G L(n, K), B \in \operatorname{Mat}_{n}(K)
$$

One easily checks that the map $\left(\begin{array}{cc}A & B \\ 0 & A\end{array}\right) \rightarrow \operatorname{tr}\left(A^{-1} B\right)$ is a group homomorphism. For any $a \in K$ let's define the representation $T_{a}$ of $G$ by

$$
T_{a}:=\operatorname{Ind}_{H}^{G} \chi_{a}\left(\operatorname{tr}\left(A^{-1} B\right)\right) .
$$

Question. Is it true that all representations of $G$ which occur in the spectrum of $T_{a}$ for $a \neq 0$ are self-dual?

Let's translate this question into orbit language. According to rule 8 of the User's Guide self-dual representations are associated to orbits with the property $\Omega=-\Omega$. As usual, we identify $\mathfrak{g}^{*}$ with $\mathfrak{g}$ via the pairing $\langle F, X\rangle=\operatorname{tr}(F X)$ and the space $\mathfrak{h}^{*}$ with the space of matrices of the form $\left(\begin{array}{ll}A & 0 \\ B & A\end{array}\right)$. Then, according to rule 4 , the spectrum of $T_{a}$ consists of representations associated to those orbits in $\mathfrak{g}^{*}$ whose projection to $\mathfrak{h}^{*}$ contains the point

$$
F_{a}=\left(\begin{array}{cc}
0 & 0 \\
a \cdot 1 & 0
\end{array}\right) \in \mathfrak{h}^{*} .
$$

It is clear that the preimage of $F_{a}$ is the set of matrices of the form

$$
\left(\begin{array}{cc}
X & Y \\
a \cdot 1 & -X
\end{array}\right), \quad \text { where } X, Y \in \operatorname{Mat}_{n}(K) .
$$

So, a positive answer to the question above corresponds to the following statement about matrices. 
Proposition 1. For any $X, Y \in \operatorname{Mat}_{n}(K)$ and any non-zero $a \in K$ the matrices $\left(\begin{array}{cc}X & Y \\ a \cdot 1 & -X\end{array}\right)$ and $\left(\begin{array}{cc}-X & -Y \\ -a \cdot 1 & X\end{array}\right)$ are similar, i.e. belong to the same conjugacy class in $\operatorname{Mat}_{2 n}(K)$.

Proof. Direct verification that the conjugation by the matrix $\left(\begin{array}{cc}-1 & 2 X / a \\ 0 & 1\end{array}\right)$ indeed does the trick.

I remind you that this "answer" to the above question has only a heuristic value, because for reductive groups over local fields the relation between coadjoint orbits and unirreps is far from clear. It was a pleasure for me to know that this "answer" was confirmed and the corresponding theorem is now proved.

5.2. Finite groups. The application of the orbit method to representation of finite groups is based on the possibility of considering some finite groups as groups of $\mathbb{F}_{q}$-points of an algebraic group $G$ defined, say, over $\mathbb{Z}$.

In the last few years I have tried to understand the simplest case where $G=G_{n}$ is the group of upper unitriangular matrices of order $n$. This has led me to some very interesting combinatorial and number-theoretic problems. I refer to the papers $[\mathrm{K} 6]$ and $[\mathrm{KM}]$ for details and mention here only two problems.

1. Is it true that any upper unitriangular $n \times n$ matrix with elements from $\mathbb{F}_{q}, q=2^{l}$, is conjugate to its inverse in the group $G_{n}$ ? As I learned recently, it has been checked to be true up to $n=12$, and a counterexample has been announced for $n=13, q=2$; see [I].

This example contradicts the "naive" character formula conjectured in [K6] but still leaves a hope that there is a correspondence between unirreps and coadjoint orbits for $G_{n}\left(\mathbb{F}_{q}\right)$ such that $\operatorname{dim} \pi_{\Omega}=\sqrt{\# \Omega}$.

Define the $\zeta$-function of a compact group $G$ as

$$
\zeta_{G}(s)=\sum_{\lambda \in \widehat{G}} d(\lambda)^{-s},
$$

where $d(\lambda)=\operatorname{dim} \pi_{\lambda}$. For a finite group this is an exponential polynomial in $s$ with

$$
\zeta_{G}(-2)=\# G, \quad \zeta_{G}(0)=\# \widehat{G} .
$$

In the case of $G_{n}\left(\mathbb{F}_{q}\right)$ the conjecture above is equivalent to the following relation between $\zeta_{G_{n}\left(\mathbb{F}_{q}\right)}(s)$ and the number $\mathcal{O}_{n, m}(q)$ of $2 m$-dimensional coadjoint orbits for $G_{n}\left(\mathbb{F}_{q}\right)$ :

$$
\zeta_{G_{n}\left(\mathbb{F}_{q}\right)}(s)=\sum_{m} \mathcal{O}_{n, m}(q) \cdot q^{-m s} .
$$

It is supported by the fact that the number of unirreps is equal to the number of coadjoint orbits and the sum of squares of their dimensions is equal to $\# G_{n}\left(\mathbb{F}_{q}\right)$. So, (5.2.3) is true at least for $s=0$ and -2 .

2. The remarkable fact is that the right hand side of (5.2.3) can be considered as a partition function for some peculiar lattice model on $\mathfrak{g}_{n}^{*}\left(\mathbb{F}_{q}\right)$ where $\operatorname{rk} B_{F}$ plays the role of the energy function and $-s$ is the analog of the inverse temperature $\beta=\frac{1}{k T}$. Still more interesting is the fact that for $s=2-2 r$, an even negative 
integer, this partition function can be written in the form

$$
\zeta_{G_{n}\left(\mathbb{F}_{q}\right)}(2-2 r)=q^{-r n(n-1)} \cdot \sum_{X, Y, F} \theta(\langle F,[X, Y]\rangle),
$$

where $X, Y$ run through $\mathfrak{g}_{n}\left(\mathbb{F}_{q^{r}}\right), F$ runs through $\mathfrak{g}_{n}^{*}\left(\mathbb{F}_{q}\right)$ naturally embedded in $\mathfrak{g}_{n}^{*}\left(\mathbb{F}_{q^{r}}\right)$ and $\theta$ is any non-trivial additive character of $\mathbb{F}_{q^{r}}$. So, the algebraic version of the inverse temperature is the degree of the extension of a finite field!

\subsection{Infinite dimensional groups.}

5.3.1 General remarks. There are practically no general theorems in this part of representation theory. But for some specific cases beautiful and deep results have been obtained (see e.g. the books $[\mathrm{Is}],[\mathrm{Ka}],[\mathrm{PS}]$ and $[\mathrm{N}]$ ). So, this is a natural domain for the application of the orbit method as was suggested already in [K2] and more thoroughly in my talk presented to the Helsinki ICM (1978). ${ }^{28}$

For the most important examples of infinite dimensional Lie groups the description of coadjoint orbits and their geometry is a very interesting and non-trivial problem which is usually connected (or even coincides) with some classical problems of global analysis and geometry. I refer to my lectures [K4] where several examples are described in detail.

5.3.2 Affine Lie algebras and loop groups. Let $K$ be a connected compact Lie group. The set $L K=C^{\infty}\left(S^{1}, K\right)$ of all smooth mappings of the circle $S^{1}$ to $K$ is an infinite dimensional Lie group (with pointwise multiplication of functions). The corresponding Lie algebra is the space $L \mathfrak{k}=C^{\infty}\left(S^{1}, \mathfrak{k}\right)$ where $\mathfrak{k}=\operatorname{Lie}(K)$ is the Lie algebra of $K$. The algebraic versions of this notion arise when we replace smooth functions on $S^{1}$ by trigonometric polynomials.

We adopt some assumptions, facts and notations from 3.1. In particular, we suppose that $K$ is simply connected and $\mathfrak{k}=\operatorname{Lie}(K)$ is simple. We fix an Adinvariant scalar product $\langle$,$\rangle on \mathfrak{k}$ normalized by the condition that the maximal root $\psi$ satisfies $\langle\psi, \psi\rangle=2$. The group $L K$ has a non-trivial central extension $\widetilde{L K}$ by a circle group $\mathbb{T}^{1}$. Moreover, the circle group of rotations of $S^{1}$ acts on $L K$ by outer automorphisms, and this action can be extended to $\widetilde{L K}$. Thus, we get the group

$$
G=\widehat{L K}=\mathbb{T}^{1} \ltimes \widetilde{L K}
$$

The corresponding Lie algebra has the form

$$
\mathfrak{g}=\widehat{L \mathfrak{k}}=\mathbb{R} \cdot D \oplus \mathbb{R}[\cos t, \sin t] \otimes \mathfrak{k} \oplus \mathbb{R} \cdot C
$$

so that an element of $\mathfrak{g}$ is a triple $(\alpha, X, \beta)$ where $\alpha$ and $\beta$ are real numbers and $X: S^{1} \rightarrow \mathfrak{k}$ is a map given in coordinates by trigonometric polynomials.

The commutation relations are

$$
\begin{aligned}
{\left[\left(\alpha_{1}, X_{1}, \beta_{1}\right),\left(\alpha_{2}, X_{2}, \beta\right)\right]=} & \left(0,-\alpha_{2} X_{1}^{\prime}+\alpha_{1} X_{2}^{\prime}+\left[X_{1}, X_{2}\right],\right. \\
& \left.\frac{1}{2 \pi} \int_{0}^{2 \pi}\left\langle X_{1}^{\prime}, X_{2}\right\rangle d t\right) .
\end{aligned}
$$

It is called real affine algebra (or real Kac-Moody algebra of affine type).

\footnotetext{
${ }^{28}$ For a political reason I was not allowed to go to the Congress (as many other Soviet mathematicians), so I missed the opportunity to discuss my ideas with Western mathematicians who became interested in infinite dimensional groups at the same time.
} 
Affine algebras share many properties of simple finite dimensional Lie algebras. E.g. they possess an Ad-invariant bilinear form $B$ given by

$$
B\left(\left(\alpha_{1}, X_{1}, \beta_{1}\right),\left(\alpha_{2}, X_{2}, \beta\right)\right)=\alpha_{1} \beta_{2}+\alpha_{2} \beta_{1}+\frac{1}{2 \pi} \int_{0}^{2 \pi}\left\langle X_{1}, X_{2}\right\rangle d t .
$$

One can associate to such an algebra an infinite root system, define the corresponding affine Weyl group and construct a series of so-called integrable irreducible representations labelled by dominant weights. We refer to [Ka] and [PS] for the detailed exposition of this theory.

All this has a nice interpretation in terms of coadjoint orbits. We give here the outline of this approach including the integral formula for the generalized characters (rule 6) which has been obtained in [Fr] and looks like a functional integral over the corresponding infinite dimensional orbit.

We start with the descriptions of coadjoint orbits. Using the invariant form (5.3.2) we can identify $\mathfrak{g}$ with a part of $\mathfrak{g}^{* 29}$ and consider the adjoint orbits. The center of $\widehat{L K}$ acts trivially. So, it is enough to consider the action of the group $\mathbb{T}^{1} \ltimes L K$ on $\mathfrak{g}$.

Theorem 1. ([Fr], $[\mathrm{PS}]$ ) The action of $L K$ on $\mathfrak{g}$ has the form

$$
A d(g)(\alpha, X, \beta)=\left(\alpha, g X g^{-1}-\alpha Y, \beta+\langle X, Y\rangle-\frac{\alpha}{2}\langle Y, Y\rangle\right)
$$

where $Y=g^{\prime} g^{-1}$.

This formula has several important implications.

1. The quantity $\alpha$ is preserved. It is called the central charge of the orbit and, as we shall see soon, the integrality condition requires it to be an integer.

2. A momentum $(\alpha, X, \beta)$ with $X=$ const is invariant under the rotation group; hence its $L K$-orbit coincides with $G$-orbit.

3 . The orbits behave differently depending on whether the central charge vanishes or not. In the former case they are just loops in the space $\mathcal{O}(G)$ of $G$-orbits in $\mathfrak{g}$ and have infinite codimension; in the latter case they are surfaces of codimension $2+l$ where $l=\operatorname{rk} K$ (see below).

4. The stabilizer of a point $(\alpha, X, \beta)$ with $\alpha \neq 0$ and $X=$ const $\in \mathfrak{t}_{\text {reg }}$ is $\mathbb{T} \times T \times \mathbb{T}$. So, there are $2+l$ integrality conditions.

Now, the description of orbits is given in two steps.

Proposition 2. Any $G$-orbit with non-zero central charge meets the subspace $\mathfrak{g}_{0} \subset$ $\mathfrak{g}$ given by the condition $X=$ const.

Proof. It follows from Floquet theory of systems of ordinary differential equations with periodic coefficients.

Let $T \cong \mathbb{T}^{l}$ be a maximal torus in $K, \mathfrak{t}$ its Lie algebra, $Q^{\vee}=\frac{1}{2 \pi i}\left(\exp ^{-1}(e) \cap \mathfrak{t}\right)$ the coroot lattice in $i \mathfrak{t}, W=N_{K}(T) / T$ the ordinary Weyl group and $W_{\text {aff }}$ the affine Weyl group, i.e. the semi-direct product of $W$ and $Q^{\vee}$. $W_{\text {aff }}$ acts naturally on $\mathfrak{t}$ by affine transformations: $(w, \gamma) \cdot X=w(X)+2 \pi i \gamma$. We denote by $S$ a fundamental domain for $W_{\text {aff }}$ in $\mathfrak{t}$. The standard coordinates on $\mathfrak{t}$ are $x_{k}(X)=\frac{2\left\langle X, \alpha_{k}\right\rangle}{i\left\langle\alpha_{k}, \alpha_{k}\right\rangle}$ where

\footnotetext{
${ }^{29}$ In the finite dimensional case the existence of an invariant form implies an isomorphism $\mathfrak{g} \cong \mathfrak{g}^{*}$. In the infinite dimensional situation $\mathfrak{g}^{*}$ is much larger than $\mathfrak{g}$, but, fortunately, the most interesting orbits usually lie inside $\mathfrak{g}$.
} 
$\alpha_{k}, 1 \leq k \leq l$, are the simple roots of $\left(\mathfrak{k}_{\mathbb{C}}, \mathfrak{t}_{\mathbb{C}}\right)$. In these coordinates $S$ can be chosen as the simplex given by the inequalities

$$
x_{k} \geq 0,1 \leq k \leq l, \quad \sum_{i=0}^{l} a_{k} x_{k} \leq 1
$$

where $a_{i}$ are the coefficients of the decomposition of the maximal root $\psi$ into linear combination of simple roots: $\psi=\sum_{i=0}^{l} a_{k} \alpha_{k}$.

Proposition 3. Any LK-orbit with non-zero central charge $\alpha$ contains a unique point $\mathcal{F}=(a, Y, b)$ with $Y=$ const $\in a S$.

Proof. It follows from the study of the coadjoint action of $g=\exp (X) \in L K$ where $X(t)$ is a linear function of the form $X(t)=X_{0}+t \cdot X_{1}(t)$ where $X_{1} \in Q^{\vee}$.

In fact, the integrality conditions imply that $a, b \in \mathbb{Z}, Y \in P$ (or, equivalently, $y_{k}=\frac{2\left\langle Y, \alpha_{k}\right\rangle}{i\left\langle\alpha_{k}, \alpha_{k}\right\rangle} \in \mathbb{Z}$ ) that leaves for $\mathcal{F}$ a finite number of possibilities for any given $a \in \mathbb{Z}$. Actually, one can establish a one-to-one correspondence between integral orbits with positive central charge and dominant weights of $\mathfrak{g}$ (hence, with integrable unirreps of $\widehat{L K}$ ).

Let $\Omega_{\Lambda}$ denote the orbit which contains $i \Lambda \in \mathfrak{g} \subset \mathfrak{g}^{*}$. As in the compact case, if we want a variant of rule 6 to hold, we have to associate a unirrep $\pi_{\Lambda}$ with the highest weight $\Lambda$ to the orbit $\Omega_{\Lambda+\tilde{\rho}}$ where $\tilde{\rho}$ is the analog of the half sum of positive roots for the affine algebra $\mathfrak{g}$.

The infinite-dimensional variant of rule 6 looks exactly like the usual one,

$$
\operatorname{tr} \pi_{\Lambda}(\exp \mathcal{X})=\frac{1}{j(\mathcal{X})} \int_{\Omega_{\Lambda+\tilde{\rho}}} e^{2 \pi i\langle\mathcal{X}, \mathcal{F}\rangle} d \mathcal{F}
$$

but needs much more explanation.

Here $\mathcal{X}=(\alpha, X, \beta)$ is supposed to be an element of $\mathfrak{g}$ while $\Lambda=(k, \lambda, n)$ with $k \in \mathbb{Z}_{+}, \lambda \in P_{+}, n \in \mathbb{Z}, \tilde{\rho}=\left(\frac{1}{2} D, \rho, 0\right)$ and $\mathcal{F}=(a, Y, b)=A d g(\Lambda+\tilde{\rho})$ belong to $\mathfrak{g}$ considered as a part of $\mathfrak{g}^{*}$.

In this case both sides of (5.3.4) taken literally do not make sense. But the point is that one can consider the continuation of the representation $\pi_{\Lambda}$ to an open domain in exp $\mathfrak{g}_{\mathbb{C}}$ defined by $\Im \alpha>0$. Representation operators in the LHS then become operators of the trace class. As for the RHS, the symplectic volume $d \mathcal{F}$ also does not make sense in the infinite-dimensional situation. But the expression $e^{2 \pi i\langle\mathcal{X}, \mathcal{F}\rangle}$ has a factor $e^{-\pi \Im \alpha\|Y\|^{2}}$, and the product $e^{-\pi \Im \alpha\|Y\|^{2}} d \mathcal{F}$ can be interpreted as a Gaussian measure on the orbit which comes from a conditional Wiener measure on the space of paths in $\mathfrak{g}$ with a given endpoint.

Finally, the function $j(\mathcal{X})$ here, as in the case of a compact group $G$, is closely related to the denominator of the Kac-Weyl character formula.

5.3.3 Virasoro algebra and Virasoro-Bott group. One of the challenging problems is to explain the rather complicated structure of the discrete series of unitary representations of the Virasoro-Bott group Vir, the central extension of Diff $+\left(S^{1}\right)$, in terms of Kähler geometry of coadjoint orbits. An attempt to do it "on a physical level" was made in $[\mathrm{AS}]$, but the problem of translation of this result into mathematical language is still open.

We recall here the basic facts concerning the beautiful geometry of coadjoint orbits for Vir. An open domain in the space vir* is covered by the orbits which are 
equivalent as a homogeneous space to the coset space $M=\operatorname{Diff}_{+}\left(S^{1}\right) / \operatorname{Rot} S^{1}$. This space has an invariant complex structure and can be described as follows.

Let $D^{+}:=\{z \in \mathbb{C}|| z \mid \leq 1\}$, and let $\mathcal{F}$ denote the space of all holomorphic functions $f$ on $D^{+}$which are univalent, ${ }^{30}$ smooth up to boundary and normalized by the conditions $f(0)=0, f^{\prime}(0)=1$.

So, if we write

$$
f(z)=z \cdot\left(1+\sum_{n \geq 1} c_{n} z^{n}\right),
$$

we can consider $\left\{c_{n}\right\}$ as coordinates on $\mathcal{F}$ which provide an embedding of this infinite dimensional manifold into $\mathbb{C}^{\infty}$.31

In fact, a function $f \in \mathcal{F}$ is uniquely defined by the simple smooth contour $K=f\left(S^{1}\right)$. The set $\mathcal{K}$ of all such contours can be considered as a geometric realization of $\mathcal{F}$. It consists of all contours $K$ around the origin such that the conformal radius of $K$ with respect to the origin is equal to 1 (the last requirement is simply a reformulation of the second normalization condition above).

The group $G=\operatorname{Diff}_{+}\left(S^{1}\right)$ acts on $\mathcal{F}$ by holomorphic transformations. Unfortunately, it is impossible to write down this action explicitly because the construction uses the Riemann uniformization theorem which gives no explicit formula. The only exception is the action of the rotation subgroup. It is given by

$$
\left(r_{\alpha} f\right)(z)=e^{-i \alpha} f\left(e^{i \alpha} z\right) \quad \text { or } \quad r_{\alpha} c_{n}=e^{i n \alpha} c_{n} .
$$

So, the stabilizer of the point $f_{0}(z)=z$ coincides with the rotation subgroup and $\mathcal{F} \cong M$ as homogeneous $G$-manifolds.

The corresponding infinitesimal action can be described explicitly.

Theorem 2. ([KY]) For any $v=v(t) \frac{d}{d t} \in V$ ect $S^{1}$ the corresponding $L_{v} \in V e c t \mathcal{F}$ is given by

$$
L_{v}(f)(z)=\frac{f^{2}(z)}{2 \pi} \oint_{S^{1}}\left(\frac{t f^{\prime}(t)}{f(t)}\right)^{2} \cdot \frac{v(t)}{(f(t)-f(z))} \cdot \frac{d t}{t} .
$$

The action of $\operatorname{Diff}_{+}\left(S^{1}\right)$ on $\mathcal{F}$ defines a two-parameter series of representations of the Lie algebra vir $=\operatorname{Vect}\left(S^{1}\right) \propto \mathbb{R}$ in the space of analytic functions on $\mathcal{F}$ by differential operators of the first order:

$$
\left(T_{c, h}(v, a) F\right)(f)=\left\{\left[L_{v}(f)+\Phi_{c, h}(v, f)+a c\right] F\right\}(f)
$$

where $\Phi(v, f)$ is linear in $c, h$ and $v \in C^{\infty}\left(S^{1}\right)$ is analytic in $f \in \mathcal{F}$ and satisfies

$$
L_{v_{1}} \Phi_{c, h}\left(v_{2}, f\right)-L_{v_{2}} \Phi_{c, h}\left(v_{1}, f\right)=\Phi_{c, h}\left(v_{1} v_{2}^{\prime}-v_{2} v_{1}^{\prime}, f\right)+\frac{c}{12} \cdot \int_{S^{1}} v_{1} v_{2}^{\prime \prime \prime} .
$$

Proposition 4. ([K8]) The explicit formula for $\Phi_{c, h}(v, f)$ has the form

$$
\Phi_{c, h}(v, f)=\oint_{S^{1}}\left[h\left(\frac{t f^{\prime}(t)}{f(t)}\right)^{2}+\frac{c t^{2}}{12} S(f)\right] \cdot v(t) \frac{d t}{t}
$$

\footnotetext{
${ }^{30}$ Univalent means that $f\left(z_{1}\right) \neq f\left(z_{2}\right)$ for $z_{1} \neq z_{2}$.

${ }^{31}$ The famous De Branges' Theorem (formerly the Bieberbach Conjecture) shows that the image in fact lies in the bounded domain $\left|c_{n}\right|<n+1$, for $n \geq 1$.
} 
where $S(f)=\frac{f^{\prime \prime \prime}}{f^{\prime}}-\frac{3}{2}\left(\frac{f^{\prime \prime}}{f^{\prime}}\right)^{2}$ is the so-called Schwarzian derivative of $f .^{32}$

Recall now some known fact from the representation theory of the algebra vir (see [Ka], [N]). Let $L_{c, h}$ be the irreducible highest weight module over vir. It is called unitarizable if one can define an inner product on it s.t. $L_{n}^{*}=L_{-n}$ and the vacuum vector $x_{0}$ has the length 1 . Such a product is unique and exists in the following cases:

a) $c \geq 1, h \geq 0$

b) $c=1-\frac{6}{m(m+1)}, \quad h=h_{p, q, m}=\frac{(m p+p-m q)^{2}-1}{4 m(m+1)}$, where $m=2,3,4, \cdots, \quad 0<q \leq p<m$.

In the first case $L_{c, h}$ coincides with the Verma module $V_{c, \mathfrak{h}}$, while in the second case it is the quotient of $V_{c, h}$ by a non-trivial submodule consisting of vectors of zero length. This submodule is generated by the so-called singular vectors annihilated by all $L_{k}, k>0$, and different from the vacuum vector. The first singular vector is on the $p q$-th level.

The simplest (and most trivial) example is $m=2$ where $p=q=1, c=h=0$ and $L_{0,0}$ is a trivial one dimensional module. It is realized in the one dimensional space of constant functions on $\mathcal{F}$.

Consider in more detail the first non-trivial case $m=3$. Here $c=\frac{1}{2}, h=$ $0, \frac{1}{2}$, or $\frac{1}{16}$ and the singular vectors are respectively on the first, second and fourth levels.

For $h=0$ the singular vector is just $L_{-1} x_{0}$. Moreover, the basis in $L_{\frac{1}{2}, 0}$ consists of vectors of the form

$$
L_{-k_{1}} L_{-k_{2}} \cdots L_{-k_{r}} x_{0}, k_{i} \geq 2 .
$$

The geometric realization of this space has a simple description: it consists exactly of functions on $\mathcal{F}$ which are constant along the leaves of a $G$-invariant foliation of $\mathcal{F}$. For all other cases the geometric description of $L_{c, h}$ is unknown.

5.3.4. Moduli space of flat G-bundles and representations of the Teichmüller group. One more source of symplectic manifolds was discovered recently (see $[\mathrm{AB}]$ ). It is the moduli space $\mathcal{M}(G, \Sigma)$ of flat connections in principal $G$-bundles over a 2-dimensional oriented manifold $\Sigma$ (Riemann surface). The symplectic structure on this space arises as a result of symplectic reduction (see 7.1) from the space $\mathcal{A}(G, \Sigma)$ of all connections with the symplectic structure defined geometrically

$$
\sigma\left(A_{1}, A_{2}\right)=\int_{\Sigma}\left(A_{1} \wedge A_{2}\right) .
$$

Here the bilinear operation $\left(A_{1} \wedge A_{2}\right)$ is constructed from the invariant bilinear form on $\mathfrak{g}=\operatorname{Lie}(G)$ and the exterior product of differential forms on $\Sigma$.

The space $\mathcal{M}(G, \Sigma)$ is almost compact (its compactification differs from the initial space by a submanifold of large codimension) and possesses a Kähler structure which depends on the complex structure on $\Sigma$. The geometric quantization of $\mathcal{M}(G, \Sigma)$ is a very interesting problem closely related with modern mathematical physics on one side and with values of the group $\zeta$-functions in integer points on the other (See [W2], [Za]).

\footnotetext{
${ }^{32}$ The Schwarzian derivative was introduced in 1869 by H.A. Schwarz in complex analysis and recently found new applications in the theory of one-dimensional dynamical systems (see e.g. $[\mathrm{De}])$.
} 
Though this example leads to a finite dimensional quantum space (the volume of $\mathcal{M}(G, \Sigma)$ is finite), the construction depends heavily on infinite dimensional considerations. (See, however, $[\mathrm{FR}]$ for finite dimensional treatment of this example.)

\subsection{Quantum groups and Poisson-Lie groups.}

5.4.1. Discrete versions of analysis. There are two different ways to make the real line $\mathbb{R}$ discrete: replace it by $h \cdot \mathbb{Z}$ (an infinite arithmetic progression with difference $h$ ) or by $q^{\mathbb{Z}}$ (an infinite geometric progression with ratio $q$ ). Correspondingly, there are two ways to replace the differential operator $\frac{d}{d x}$ by a difference operator:

$$
\left.\left(D_{h}^{\text {add }} f\right)(x):=\frac{f(x+h)-f(x)}{h} \quad \text { (or, symmetrically, } \frac{f(x+h)-f(x-h)}{2 h}\right) .
$$

This way goes back to Euler and leads to the beautiful calculus of finite differences.

The second way goes back to Gauss and plays a basic role in the theory of quantum groups. Here the differential operator $\frac{d}{d x}$ is replaced by a difference operator:

$$
\left.\left(D_{q}^{\text {mult }} f\right)(x):=\frac{f(q x)-f(x)}{q x-x} \quad \text { (or, symmetrically, } \quad \frac{f(q x)-f\left(q^{-1} x\right)}{q x-q^{-1} x}\right) .
$$

If we denote by $T_{q}$ the operator of dilation by $q$, then the symmetric version of $D_{q}^{\text {mult }}$ can be written as

$$
D_{q}^{\text {mult }}=x^{-1} \cdot \frac{T_{q}-T_{q}^{-1}}{q-q^{-1}} .
$$

The obvious commutation relation $T_{q} \cdot x=q x \cdot T_{q}$ implies the relations

$$
x \cdot D_{q}^{\text {mult }}=\frac{T_{q}-T_{q}^{-1}}{q-q^{-1}}, \quad D_{q}^{\text {mult }} \cdot x=\frac{q T_{q}-q^{-1} T_{q}^{-1}}{q-q^{-1}} .
$$

5.4.2 Quantum groups. There are already about ten books devoted to the exposition of this very fashionable subject, and I have no wish to compete with them. But I want to express here my own point of view on the appearance of quantum groups.

We start with the classical Lie algebra $\mathfrak{g l}(n, \mathbb{R})$. This algebra can be realized by differential operators of the form $\sum_{i, j=1}^{n} a_{i, j} x_{i} \partial_{j}$ and has the natural basis

$$
e_{i, j}=x_{i} \partial_{j}, \quad 1 \leq i, j \leq n .
$$

Define the discrete analog of these operators by

$$
E_{i, j}=x_{i} D_{j}, \quad 1 \leq i, j \leq n,
$$

where $D_{j}$ is the operator $D_{q}^{\text {mult }}$ w.r.t. the variable $x_{j}$.

The linear span of operators $E_{i, j}$ is no longer a Lie algebra. To describe the commutation properties of the resulting set of operators we consider the basic operators

$$
E_{i}:=E_{i, i+1}, \quad F_{i}:=E_{i+1, i}, \quad 1 \leq i \leq n-1 .
$$

Their classical prototypes $\left\{e_{i}, f_{i}, \quad 1 \leq i \leq n-1\right\}$ generate the Lie algebra $\mathfrak{s l}(n, \mathbb{R})$ and satisfy the relations:

$$
\begin{gathered}
{\left[e_{i}, e_{j}\right]=\left[f_{i}, f_{j}\right]=0 \quad \text { for }|i-j| \geq 2, \quad\left[e_{i}, f_{j}\right]=\delta_{i, j} h_{i}} \\
\left(\operatorname{ad} e_{i}\right)^{2} e_{j}=e_{i}^{2} e_{j}-2 e_{i} e_{j} e_{i}+e_{j} e_{i}^{2}=0, \quad\left(\operatorname{ad} f_{i}\right)^{2} f_{j}=f_{i}^{2} f_{j}-2 f_{i} f_{j} f_{i}+f_{j} f_{i}^{2}=0
\end{gathered}
$$

where $h_{i}=e_{i, i}-e_{i+1, i+1}, 1 \leq i \leq n-1$. 
The corresponding discrete operators $\left\{E_{i}, F_{i}, \quad 1 \leq i \leq n-1\right\}$ satisfy the $q$-analog of these relations:

$$
\begin{gathered}
{\left[E_{i}, E_{j}\right]=\left[F_{i}, F_{j}\right]=0 \text { for }|i-j| \geq 2, \quad\left[E_{i}, F_{j}\right]=\delta_{i, j} \frac{K_{i}-K_{i}^{-1}}{q-q^{-1}}} \\
E_{i}^{2} E_{j}+E_{j} E_{i}^{2}=\left(q+q^{-1}\right) E_{i} E_{j} E_{i}, \quad F_{i}^{2} F_{j}+F_{j} F_{i}^{2}=\left(q+q^{-1}\right) F_{i} F_{j} F_{i}
\end{gathered}
$$

where $K_{i}=T_{i} / T_{i+1}$ and $T_{i}$ is the dilation operator with respect to the $i$-th variable. One can easily check that these quantum relations become the classical ones when $q=1$.

The associative algebra $U_{q}(\mathfrak{s l}(n, \mathbb{R}))$ is by definition generated by the elements $E_{i}, F_{i}, K_{i}, K_{i}^{-1}, 1 \leq i \leq n$, satisfying the relations above. It is called the quantum analog of the universal enveloping algebra $U(\mathfrak{s l}(n, \mathbb{R}))$.

The main property of an enveloping algebra of a Lie algebra $\mathfrak{g}$ is that it is a variant of the group algebra for the corresponding Lie group $G$. Namely, it is the space of all distributions on the group with support $\{e\}$. As such it possesses an additional algebraic structure, the comultiplication

$$
\Delta: U(\mathfrak{g}) \otimes U(\mathfrak{g}) \rightarrow U(\mathfrak{g})
$$

which is dual to the multiplication of test functions on $G$.

It turns out that $U_{q}(\mathfrak{s l}(n, \mathbb{R}))$ also admits a comultiplication which looks like

$$
\Delta E_{i}=E_{i} \otimes 1+K_{i} \otimes E_{i}, \quad \Delta F_{i}=F_{i} \otimes K_{i}^{-1}+1 \otimes F_{i} .
$$

This operation is not cocommutative, which means that the function algebra on a quantum group should be non-commutative. So, quantum groups have no points in the usual sense and cannot be considered as sets. But still the analogy with ordinary groups is rather useful and suggestive.

5.4.3 Poisson-Lie groups. Here we introduce the notion which replaces the space $\mathfrak{g}^{*}$ in the quantum case.

The first approximation to a quantum group is a so-called Poisson-Lie group which is an ordinary Lie group $G$ with an additional structure: the Poisson brackets in the space of functions on $G$. It arises when we consider the (non-commutative) multiplication in the function algebra on a quantum group as a deformation of the ordinary multiplication in the function algebra on $G$.

Namely, this deformation has the form

$$
f \circ g=f g+h\{f, g\}+o(h),
$$

where $h$ is the deformation parameter (considered either as a real number or as a formal variable) and $\{$,$\} is the Poisson bracket on G$ compatible with group multiplication.

In terms of the corresponding bivector $c$ compatibility means

$$
c(x y)=L_{x}^{*} c(y)+R_{y}^{*} c(x),
$$

where $L_{x}, R_{y}$ are the operators of left and right shifts on $G$. It follows that $c$ vanishes at the unit element $e \in G$ and is completely determined by its first order jet at $e$. The latter gives a Lie algebra structure $[,]_{*}$ on the space $T_{e}^{*}(G)=\mathfrak{g}^{*}$.

Proposition 5. The two Lie algebras $\mathfrak{g}$ and $\mathfrak{g}^{*}$ satisfy in turn a compatibility condition which can be formulated in three equivalent ways:

a) $\varphi: \mathfrak{g}^{*} \rightarrow \mathfrak{g}^{*} \times \mathfrak{g}^{*}$ is a 1-cocycle on $\mathfrak{g}^{*}$ with values in $\wedge^{2} \mathfrak{g}^{*}$ (here $\varphi$ is the map dual to the commutator in $\mathfrak{g})$; 
b) $\psi: \mathfrak{g} \rightarrow \mathfrak{g} \times \mathfrak{g}$ is a 1-cocycle on $\mathfrak{g}$ with values in $\wedge^{2} \mathfrak{g}$ (here $\psi$ is the map dual to the commutator in $\left.\mathfrak{g}^{*}\right)$;

c) the space $\mathfrak{a}:=\mathfrak{g} \oplus \mathfrak{g}^{*}$ has a Lie algebra structure which preserves the natural symmetric bilinear form on $\mathfrak{a}$ and extends the Lie algebra structures on $\mathfrak{g}$ and $\mathfrak{g}^{*}$ given above.

The last formulation suggested by $\mathrm{Yu}$. I. Manin is the most convenient one. The $\operatorname{data}\left(\mathfrak{a}, \mathfrak{g}, \mathfrak{g}^{*}\right)$ has the name of

Manin triple. The category of Manin triples is naturally isomorphic to the category of simply connected Poisson-Lie groups.

We see that $\mathfrak{g}$ and $\mathfrak{g}^{*}$ play symmetric roles in the definition of a Manin triple. It follows that to every simply connected Poisson-Lie group $G$ there corresponds a dual Poisson-Lie group $G^{*}$.

5.4.4. Quantum version of the orbit method. The relation between the notion of Poisson-Lie group and the orbit method is based on the following simple observation.

Proposition 5. Let $G$ be a Lie group with the trivial (zero) Poisson bracket. Then the dual Poisson-Lie group $G^{*}$ is commutative and coincides with $\mathfrak{g}^{*}$ endowed with the canonical Lie-Berezin Poisson structure.

So, $G^{*}$ is a direct analog of $\mathfrak{g}^{*}$ and can be considered as a quasi-classical approximation to the quantum version of $\mathfrak{g}^{*}$.

One can define the infinitesimal action of $G$ on $G^{*}$, i.e. Lie algebra homomorphism $\mathfrak{g} \rightarrow \operatorname{Vect}\left(G^{*}\right)$. Consider a simply connected Lie group $A$ corresponding to $\mathfrak{a}$. It contains $G$ and $G^{*}$ as subgroups, and the image of the product map

$$
G \times G^{*} \rightarrow A, \quad\left(g, g^{*}\right) \mapsto g g^{*}
$$

contains a neighborhood of $G \cup G^{*} \subset A$. It follows that for a given $g^{*} \in G^{*}$ there exists a neighborhood $V$ of $e$ in $G$ such that for $g \in V$ the product $g g^{*}$ can be rewritten as

$$
g g^{*}=x^{*}\left(g, g^{*}\right) \cdot y\left(g, g^{*}\right), \quad x^{*} \in G^{*}, y \in G .
$$

The correspondence $g \rightarrow\left(g^{*} \rightarrow x^{*}\left(g, g^{*}\right)\right)$ defines a local action of $G$ on $G^{*}$. Usually this action is not global, but the corresponding infinitesimal action is welldefined.

Example 1. We shall describe all possible Lie-Poisson structures on the groups $S U(2)$ and $S L(2, \mathbb{R})$.

Let $\mathfrak{g}$ be a simple real Lie algebra of dimension 3 ; i.e. $\mathfrak{g}$ is either $\mathfrak{s u}(2)$ or $\mathfrak{s l}(2, \mathbb{R})$. In both cases the cohomology group $H^{1}\left(\mathfrak{g}, \wedge^{2} \mathfrak{g}\right)$ is trivial and the cocycle $\psi$ from Proposition 5 is a coboundary: $\psi(X)=\operatorname{ad} X(P \wedge Q)$.

We shall use the following property of our Lie algebra $\mathfrak{g}$ : the map $\wedge^{2} \mathfrak{g} \rightarrow \mathfrak{g}$ : $X \wedge Y \mapsto[X, Y]$ is an isomorphism of $\mathfrak{g}$-modules. Hence, the Lie algebra structure on $\mathfrak{a}$ is defined by the element $R=[P, Q]$, and direct computations show it has the form

$$
\left[X^{*}, Y^{*}\right]=([R,[X, Y]])^{*}, \quad\left[X, Y^{*}\right]=[X, Y]^{*}+[[X, R], Y] .
$$

(Here $X, Y \in \mathfrak{g}$, and we use the notation $X^{*}:=\langle X, \cdot\rangle \in \mathfrak{g}^{*}$.)

One can check that the resulting Manin triple $\left(\mathfrak{a}, \mathfrak{g}, \mathfrak{g}^{*}\right)$ depends only on the projective type of $R \in \mathfrak{g}$ (supposed to be non-zero) which is unique in case $\mathfrak{g}=\mathfrak{s u}(2)$ 
and can be elliptic, hyperbolic or parabolic in case $\mathfrak{g}=\mathfrak{s l}(2, \mathbb{R})$. Note also that $R$ can be reconstructed from $\left(\mathfrak{a}, \mathfrak{g}, \mathfrak{g}^{*}\right)$ as a basic vector in the 1-dimensional space $\left[\mathfrak{g}^{*}, \mathfrak{g}^{*}\right]^{\perp} \subset \mathfrak{g}$.

It is remarkable that the Lie algebra $\mathfrak{g}^{*}$ is always isomorphic to a subalgebra of aff $(2, \mathbb{R})$ generated by translations and dilations. One can see it from explicit realizations of the corresponding Manin triples given below. Here $x, y, z$ and $a, b, c$ are dual coordinates in $\mathfrak{g}$ and $\mathfrak{g}^{*}$.

Case 1. Put $\mathfrak{a}=\mathfrak{s l}(2, \mathbb{C})$ endowed by the bilinear form $\langle X, Y\rangle=\Im \operatorname{tr}(X Y)$, while the subalgebras $\mathfrak{g}, \mathfrak{g}^{*}$ and the element $R$ look like $\left(\begin{array}{cc}i x & y+i z \\ -y+i z & -i x\end{array}\right), x, y, z \in \mathbb{R}$; $\left(\begin{array}{cc}a / 2 & 0 \\ b-i c & -a / 2\end{array}\right), a, b, c \in \mathbb{R} ; R=\left(\begin{array}{cc}i & 0 \\ 0 & -i\end{array}\right)$.

In this case we have a global unique decomposition $A=G \cdot G^{*}$ which defines a global action of $G$ on $G^{*}$.

Case $2_{\text {ell }}$. Here $\mathfrak{a}, \mathfrak{g}^{*}$ and $R$ are the same as in case 1 , while $\mathfrak{g} \approx \mathfrak{s u}(1,1)$ consists of matrices

$$
\left(\begin{array}{cc}
i z & x+i y \\
x-i y & -i z
\end{array}\right), x, y, z \in \mathbb{R} .
$$

Case $2_{\text {hyp }}$. Put $\mathfrak{a}=\mathfrak{s l}(2, \mathbb{R}) \oplus \mathfrak{s l}(2, \mathbb{R})$ with the bilinear form

$$
\left\langle\left(X_{1}, Y_{1}\right),\left(X_{2}, Y_{2}\right)\right\rangle_{\mathfrak{a}}=\operatorname{tr}\left(X_{1} X_{2}\right)-\operatorname{tr}\left(Y_{1} Y_{2}\right) ;
$$

$\mathfrak{g}$ is $\mathfrak{s l}(2, \mathbb{R})$ diagonally embedded in $\mathfrak{a}$; it consists of elements

$$
\left(\left(\begin{array}{cc}
x & y+z \\
y-z & -x
\end{array}\right) \oplus\left(\begin{array}{cc}
x & y+z \\
y-z & -x
\end{array}\right)\right), \quad x, y, z \in \mathbb{R}
$$

$\mathfrak{g}^{*}$ and $R$ are defined by

$$
\left(\left(\begin{array}{cc}
a / 4 & (b-c) / 2 \\
0 & -a / 4
\end{array}\right) \oplus\left(\begin{array}{cc}
-a / 4 & 0 \\
(b+c) / 2 & a / 4
\end{array}\right)\right), \quad x, y, z \in \mathbb{R} ; \quad R=\left(\begin{array}{cc}
1 & 0 \\
0 & -1
\end{array}\right) .
$$

Case $2_{\text {par. }}$. Put $\mathfrak{a}=\mathfrak{s l}(2, \mathbb{R}) \ltimes \mathfrak{s l}(2, \mathbb{R})$ (semidirect product of $\mathfrak{s l}(2, \mathbb{R})$ with an abelian ideal isomorphic to $\mathfrak{s l}(2, \mathbb{R})$ as an $\mathfrak{s l}(2, \mathbb{R})$-module $)$ with the bilinear form

$$
\left\langle\left(X_{1} \ltimes Y_{1}\right),\left(X_{2} \ltimes Y_{2}\right)\right\rangle_{\mathfrak{a}}=\operatorname{tr}\left(X_{1} Y_{2}\right)+\operatorname{tr}\left(Y_{1} X_{2}\right) ;
$$

$\mathfrak{g}, \mathfrak{g}^{*}$ and $R$ look like

$$
X \ltimes 0, X \in \mathfrak{s l}(2, \mathbb{R}) ; \quad[R, X] \ltimes X, X \in \mathfrak{s l}(2, \mathbb{R}) ; \quad R=\left(\begin{array}{ll}
0 & 1 \\
0 & 0
\end{array}\right) .
$$

The product $G \cdot G^{*}$ in cases $2_{\text {ell }}$ and $2_{\text {hyp }}$ covers only a part of $A$, and the action of $G$ on $G^{*}$ is not globally defined.

The relation between $G$-orbits in $G^{*}$ and unirreps of the corresponding quantum group $G_{q}$ is very intriguing and has only started to be studied.

5.5. Groups related to von Neumann factors of type II. The beauty and richness of applications of symmetries related to root systems urged mathematicians to look for the infinite dimensional analog of these phenomena. The very successful attempt was made by V. Kac and R. Moody. They discovered a new class of infinite dimensional Lie algebras related to affine root systems. Later Kac and Peterson developed the corresponding group theory. All this is clearly exposed in [Ka].

But one has a feeling that a continuous analog of this theory should exist. To construct this analog it is natural to use the standard von Neumann factor of 
type $I I_{1}$ as a continuous analog of a matrix algebra. Very interesting problems arise in connection with the right continuous analogs of such notions as triangular subalgebra, Iwasava decomposition, flag manifold etc. ...

\section{EXPLANATION OF WHY THE ORBIT METHOD WORKS}

Some people consider the orbit method as a miracle which cannot be explained by natural arguments. Nevertheless, there exist two independent "explanations" of this phenomenon. We discuss below these arguments.

6.1. Mathematical argument. The idea behind this argument goes back to the simple but important observation that for any matrix Lie group $G$ the curved group manifold $G \subset \operatorname{Mat}_{n}(\mathbb{R})$ becomes just a linear subspace after the logarithm map

$$
G \ni g \mapsto \log g:=\sum_{k \geq 1} \frac{(-1)^{k-1}(g-1)^{k}}{k} .
$$

This map, unlike its inverse, the exponential map, is well defined only in some neighborhood of the unit element. But for the moment we leave aside this relatively small inconvenience. The more important fact is that the group law is not linearized by the logarithm map. It is given by the famous Campbell-Hausdorff formula ${ }^{33}$

$$
\log (\exp (X) \cdot \exp (Y))=X+Y+\frac{1}{2}[X, Y]+\frac{1}{12}([X,[X, Y]]+[Y,[Y, X]])+\cdots
$$

Of course, it could not be otherwise, bearing in mind that the group law is noncommutative. But already Dedekind pointed out the remedy. He observed that for non-commutative groups elements $g_{1} g_{2}$ and $g_{2} g_{1}$ can be different but always belong to the same conjugacy class. So, on the level of conjugacy classes, the group law becomes commutative again. This fact is crucial in the representation theory of finite groups and also is a foundation of the Cartan - Gelfand - Godement - HarishChandra theory of spherical functions. A new aspect of this phenomenon was observed rather recently in [WD]. It turned out that the logarithm map intertwines two kinds of convolution operations: the group convolution of class functions on $G$ and the Lie algebra convolution of $A d G$-invariant functions on $\mathfrak{g}$. In fact, the precise formulations are given in [WD] only for compact and nilpotent Lie groups (the latter can apparently be extended to the case of exponential Lie groups). In both cases the statements are essentially equivalent to the integral formula for characters (see rule 6 of the User's Guide in section 2 and subsection 3.2), though quite another technique is used for the proof in the nilpotent case.

The appearance of coadjoint orbits is now very natural: $A d G$-invariant functions on $\mathfrak{g}$ and $K(G)$-invariant functions on $\mathfrak{g}^{*}$ form the so-called dual hypergroups.

I mention also the other, infinitesimal way to express the same result. The generalized Fourier transform $\left(2.3 .4^{\prime}\right)$ also simplifies the infinitesimal characters, i.e. the action of Laplace-Casimir operators. The result can be roughly formulated as follows:

\footnotetext{
${ }^{33}$ In fact, only the existence of such a formula was proven by E. Campbell and F. Hausdorff, and the beautiful (but not very practical) explicit expression for the coefficients was found later by E. B. Dynkin.
} 
Proposition 1. After restriction to class functions and after conjugation by the operator of multiplication by $j(X)$, all Laplace-Casimir operators become differential operators with constant coefficients (in the canonical coordinates).

For semisimple Lie groups a theorem of this sort was first obtained in the thesis of F. A. Berezin in the 60's, and for the general case it was suggested in my course at IHP in 1968 and proved by M. Duflo [Du2] and V.A. Ginzburg [G].

6.2. Physical argument. This argument is now widely known under the rather lucky name of geometric quantization. The idea is to use a correspondence between classical and quantum physical systems. As we understand now, there is no canonical and universal correspondence: the quantum world is different from the classical one. Nonetheless, for many particular systems quantization rules which allow one to construct a quantum system from the classical one were formulated. Moreover, the symmetry possessed by a classical system is usually inherited by the quantum counterpart.

Let's now consider the elementary systems with a given symmetry group $G$ (that is, a system which cannot be decomposed into smaller parts without breaking the symmetry).

On the classical level the phase space of such a system should be a homogeneous symplectic $G$-manifold $M$. On the quantum level this is an irreducible unitary representation of $G$ in some Hilbert space $\mathcal{H}$. It seems that the quantization principle suggests a correspondence between homogeneous symplectic $G$-manifolds on the one side and unirreps of $G$ on the other.

Actually, the situation is slightly more delicate. It is known that the energy function for classical systems is defined up to an additive constant, while for a quantum system the energy is uniquely defined and is usually non-negative. This shows that the right classical counterpart to quantum systems with the symmetry group $G$ are Poisson $G$-manifolds rather than symplectic ones. But we have seen in 1.5 that homogeneous Poisson $G$-manifolds are essentially coadjoint orbits. So we come to the desired correspondence between unirreps and coadjoint orbits.

In conclusion I would like to quote a sentence from the recent preprint "Deformation quantization" by Kontsevich (cf. [Kon]):

Now we can say finally that the orbit method has solid background.

\section{BYPRODUCTS, SIDE EFFECTS AND RELATED TOPICS}

The orbit method stimulated the study of coadjoint orbits and turned out to be related to several other domains which were rapidly developing in recent decades. We briefly discuss here the three most important directions.

7.1. The moment map. The first general definition of the moment map was given by J.-M. Souriau [S], although its particular cases (e.g. related to the Galilei and Poincaré groups) were known to physicists long ago. In particular, the famous E. Noether theorem describing the connection between symmetries and invariants is simply the moment map for a one-dimensional Lie group of symmetries.

Most of the new applications of the moment map are related to the notion of symplectic reduction. This procedure, which also goes back to classical Hamiltonian mechanics, is naturally formulated in terms of the moment map. Let $G$ be a connected Lie group, $(M, \sigma)$ a symplectic $G$-Poisson manifold and $\mu: M \rightarrow \mathfrak{g}^{*}$ the associated moment map. For any coadjoint orbit $\Omega \subset \mathfrak{g}^{*}$ the set $M_{\Omega}=\mu^{-1}(\Omega)$ 
is $G$-invariant. Suppose that this set is a smooth manifold and that $G$ acts on it so that all orbits have the same dimension. ${ }^{34}$ Then the set $M_{\Omega} / G$ of $G$-orbits in $M_{\Omega}$ is also a smooth manifold and possesses a canonical symplectic structure. (One can easily check that the restriction of $\sigma$ to $M_{\Omega}$ is degenerate and $\left.\operatorname{ker} \sigma\right|_{M_{\Omega}}$ at the point $m$ coincides with the tangent space to the $G$-orbit of $m$.) This procedure allows us to reduce the study of a mechanical system with the symmetry group $G$ to the study of another system with less degrees of freedom. Sometimes, it is worthwhile to reverse this procedure and consider a complicated low-dimensional system as a result of the reduction of a simple higher-dimensional system.

We refer to $[\mathrm{AG}]$ for a survey of the symplectic geometry and its applications.

7.2. Geometric quantization. This is one possible way to translate into mathematical language the physical term "quantization" (some others are algebraic, asymptotic, deformational, path-integral quantization, etc.). All these theories are based on the premise that classical and quantum mechanics are just different realizations of the same abstract scheme.

The goal of geometric quantization is to construct quantum objects from the geometry of classical ones. Historically, there were two sources of this approach:

1) the "quantization rules" of old quantum mechanics which become more and more elaborate (but still remain adjusted to rather special Hamiltonians defined on special phase spaces),

2) the functor of unitary induction and its generalizations in the representation theory which allowed one to construct explicitly the unitary duals (or at least a large part of it) for many Lie groups.

It was B. Kostant who observed in [Ko1] that one can merge these theories into a new one. Since then geometric quantization became very popular, especially among physicists. We have, however, to remark that practically no general results in the non-homogeneous situation were obtained. The quantization rules mentioned above are usually not well defined and sometimes even contradictory. In the homogeneous situation they are practically equivalent to one or another variant of the inducing procedure (see section 2 and below). We refer to [K5], [Ko1], [So] for the definition and basic properties of geometric quantization.

The most interesting applications of geometric quantization are related to infinite dimensional systems (see e.g. [W2]). Many of them are only proved "on the physical level". See [DKN], [AS], [GS], [V] for further details.

7.3. Integrable systems. This huge domain was intensively developed during the last 30 years. Before that isolated examples were known, and no general theory existed. The new era began with the seminal discovery that the so-called Kortewegde Vries (KdV for short) equation

$$
p_{t}=p p_{x}+p_{x x x}
$$

is a completely integrable system which possesses an infinite series of conservation laws. Since then a lot of important examples of classical and quantum integrable systems have been found and several schemes were proposed to explain their appearance (see e.g. $[\mathrm{DKN}])$.

\footnotetext{
${ }^{34}$ In practical situations these conditions are often violated on a submanifold of lower dimension. Then one has to delete this submanifold or consider manifolds with singularities.
} 
The orbit method is a natural source of homogeneous symplectic manifolds (coadjoint orbits) which can be considered as phase spaces of classical mechanical systems. Note that most of them are not isomorphic to cotangent bundles and therefore do not correspond to a traditional mechanical system. On the other hand, this new kind of phase space includes the following remarkable

Example 1. Consider a 2-dimensional sphere $S^{2}$ with a symplectic form such that the total volume is an integer $n \geq 1$. As a coadjoint orbit for $\mathfrak{s u}(2)$ it admits a quantization via the corresponding unirrep of dimension $n$. The physical interpretation of this quantum system is a particle of spin $s=\frac{n-1}{2}$ which has no other degree of freedom. So we get a classical counterpart of the notion of spin which for a long time was not believed to exist.

To construct an integrable system, we need a big family of Poisson commuting functions in the classical picture or a big family of commuting operators in the quantum picture. The so-called Adler-Kostant scheme (see [Ko3] and [RS]) provides such a family. The simplest version of this scheme is based on the decomposition of a Lie algebra $\mathfrak{g}$ into a direct sum of subspaces $\mathfrak{g}_{ \pm}$which are in fact subalgebras in $\mathfrak{g}$. In this case we can define on $\mathfrak{g}$ the new commutator

$$
[X, Y]^{\sim}:=\left[X_{+}, Y_{+}\right]-\left[X_{-}, Y_{-}\right]
$$

where $X_{ \pm}$denotes the projection of $X \in \mathfrak{g}$ to $\mathfrak{g}_{ \pm}$. The commutator (7.2.1) defines a new Lie algebra structure on $\mathfrak{g}$ and a new Poisson structure on $\mathfrak{g}^{*}$.

The remarkable fact is that all functions from $P\left(\mathfrak{g}^{*}\right)^{G}$ form a Poisson commuting family with respect to this new structure. Moreover, the Hamiltonian systems corresponding to $H \in P\left(\mathfrak{g}^{*}\right)^{G}$ admit the explicit description in terms of the factorization problem:

$$
G \ni g=g_{+} \cdot g_{-}, \quad g_{ \pm} \in G_{ \pm}
$$

where $G_{ \pm}$is the Lie subgroup in $G$ corresponding to the Lie subalgebra $\mathfrak{g}_{ \pm}$. Namely, the trajectory of a point $F \in \mathfrak{g}^{*}$ under the Hamiltonian flow defined by $H \in P\left(\mathfrak{g}^{*}\right)^{G}$ is given by

$$
F(t)=K\left(g_{ \pm}(t)\right) F, \quad \text { where } g_{ \pm}(t) \text { are defined by } \quad \exp (t d H(F))=g_{+}(t)^{-1} g_{-}(t) \text {. }
$$

Application of this scheme to different Lie algebras $\mathfrak{g}$ and different points $F \in \mathfrak{g}^{*}$ gives a uniform construction for most known integrable systems.

The only deficiency of this approach is that it appeared post factum, when almost all interesting examples were discovered by other methods.

\section{Some OPEn PROBlems AND SUBJECTS FOR MEDITATION}

8.1. Functional dimension. It is well known that:

1) All (separable, infinite dimensional) Hilbert spaces are isomorphic.

2) The spaces $C^{\infty}(M)$ are isomorphic Frechet spaces for all smooth manifolds $M$ (of positive dimension).

3) All infinite countable sets are equivalent.

But there is no natural isomorphism between

1) $L^{2}(\mathbb{R}, d x)$ and $L^{2}\left(\mathbb{R}^{2}, d x d y\right)$.

2) $C^{\infty}(\mathbb{R})$ and $C^{\infty}\left(\mathbb{R}^{2}\right)$.

3) $\mathbb{Z}$ and $\mathbb{Z}^{2}$. 
The non-formal problem is to define the functional dimension $f$-dim of an infinite-dimensional space so that, for example, we have

$$
f-\operatorname{dim} L^{2}\left(\mathbb{R}^{n}, d^{n} x\right)=f-\operatorname{dim} C^{\infty}\left(\mathbb{R}^{n}\right)=n .
$$

In order to do it we have to restrict the set of morphisms between our spaces and allow only natural morphisms. There are several possible ways to impose such a restriction. One way is to define some "basic" morphisms and consider as natural only those morphisms which are compositions of basic ones. For the spaces of smooth functions on manifolds the set of basic morphisms should include:

a) multiplication by non-vanishing functions,

b) diffeomorphisms of the underlying manifolds,

c) some integral transformations such as Fourier or Radon transforms.

Another way is to introduce an additional structure in our linear spaces and consider only morphisms that preserve this structure. For example, it is not difficult to show that a compact smooth manifold $M$ is completely determined by the associative algebra $A=C^{\infty}(M)$ or by the Lie algebra $L=\operatorname{Vect}(M)$ of smooth vector fields on $M$. Indeed, the points of $M$ correspond to maximal ideals in $A$ or to Lie subalgebras of minimal codimension in $L$.

Here we discuss the related non-formal problem:

Show that if $\operatorname{dim} M_{1}>\operatorname{dim} M_{2}$, then the Lie algebra Vect $\left(M_{1}\right)$ is, firstly, "bigger" and, secondly, "more non-commutative" than Vect $\left(M_{2}\right)$.

The answer to the simplest version of the first question was obtained in [KK] and $[\mathrm{KKM}]$. Let $\xi, \eta$ be a pair of vector fields on $M$. Consider the Lie subalgebra $L(\xi, \eta) \subset \operatorname{Vect}(M)$ generated by these fields. It is a bigraded Lie algebra of the form

$$
L(\xi, \eta)=F L(x, y) / I(\xi, \eta)
$$

where $F L(x, y)$ is a naturally bigraded free Lie algebra with two generators $x, y$ and $I(\xi, \eta) \subset F L(x, y)$ is the kernel of the map $\phi: F L(x, y) \rightarrow L(\xi, \eta)$ defined by $\phi(\xi)=x, \phi(\eta)=y$. It turns out that for generic $\xi, \eta$ the ideal $I(\xi, \eta)$ depends only on $\operatorname{dim} M$. So, for each $n \in \mathbb{N}$ we get a distinguished bigraded ideal $I_{n} \subset F L(x, y)$, and we define the bigraded Lie algebra $L(n):=F L(x, y) / I_{n}$.

The growth of the numbers $a_{k . l}(n):=\operatorname{dim} L^{k, l}(n)$ can be considered as characteristic of the size of $\operatorname{Vect}(M)$ for $n$-dimensional $M$.

Theorem. (Conjectured in $[\mathrm{KK}]$, proved later by A.I. Molev)

$$
a_{k, l}(1)=p_{k}(k+l-1)+p_{l}(k+l-1)-p(k+l-1)
$$

where $p(n)$ is the standard partition function and $p_{k}(n)$ is the number of partitions of $n$ into $\leq k$ parts (or in parts of size $\leq k$ ).

The interesting corollary is that the sequence $a_{m}(n)=\sum_{k+l=m} a_{k . l}(n)$ for $n=1$ has intermediate, or subexponential growth. Namely,

$$
a_{m}(1) \approx \frac{1}{4 \sqrt{3}} e^{\pi \sqrt{\frac{2}{3} m}} \quad \text { which implies } \quad \log \log a_{m}(1) \approx \frac{1}{2} \log m .
$$

A more general result has been obtained by A.I. Molev:

$$
\log \log a_{m}(n) \approx \frac{n}{n+1} \log m .
$$

Hence, all Lie algebras of vector fields on smooth manifolds have intermediate growth. In particular, they never contain a free Lie subalgebra which has exponential 
growth:

$$
\sum_{k+l=m} \operatorname{dim} F L^{k, l}(x, y)=\frac{1}{m} \sum_{d \mid m} \mu(d) 2^{m / d} \approx \frac{2^{m}}{m} .
$$

Many interesting questions arise in connection with the second question, i.e. with the structure of algebras $L(n)$ and ideals $I(n)$. We mention only the

Conjecture. The ideal I(1) is spanned by expressions

$$
\sum_{s \in S_{4}} \operatorname{sgns} \cdot \operatorname{ad}\left(x_{s(1)}\right) \operatorname{ad}\left(x_{s(2)}\right) \operatorname{ad}\left(x_{s(3)}\right) \operatorname{ad}\left(x_{s(4)}\right) y, x_{i}, y \in L(1) .
$$

In conclusion we repeat the main problem:

Give a definition of the functional dimension of a representation so that rule 9 is valid.

8.2. Infinitesimal characters. The general proof of the modified rule 7 was obtained independently in [Du2] and [Gi]. The proof is rather involved and analytic in nature. But the statement itself is purely algebraic and certainly can be proved algebraically. My own attempt to do it was broken by the discovery that the manifold $A_{n}$ of the structure constants of $n$-dimensional Lie groups turned out to be highly reducible (the number of irreducible components grows at least as $p(n)$ ). Another approach was suggested in $[\mathrm{KV}]$, but the problem is still open.

Quite recently I learned that M. Kontsevich has found a new proof suggested by the string theory and based on the computation of a functional integral (see [Kon]). Probably this is the right solution to the problem (cf. the quotation in the end of section 6).

8.3. Multiplicities and geometry. Let $G$ be a compact simply connected Lie group and $T$ its maximal connected abelian subgroup, $\mathfrak{g} \supset \mathfrak{t}$ the corresponding Lie algebras which are identified with their duals via an $A d(G)$-invariant scalar product. Let $P \subset i \mathrm{t}^{*}$ be the weight lattice, $\rho \in P$ the sum of the fundamental weights and $\Omega_{\lambda} \subset \mathfrak{g}^{*}$ the coadjoint orbit of the point $i \lambda \in \mathfrak{t}^{*} \subset \mathfrak{g}^{*}$. We denote by $p$ the natural projection of $\mathfrak{g}^{*}$ on $\mathfrak{t}^{*}$.

It is known that for any $\lambda \in P_{+}$the set $C_{\lambda}=p\left(\Omega_{\lambda+\rho}\right)$ is the convex hull of $|W|$ different points $\{i w(\lambda+\rho), w \in W\}$. Let us call elementary cell in $\mathfrak{t}^{*}$ the set $C_{0}=p\left(\Omega_{\rho}\right)$ as well as all its translations by elements of $P$. One can check that $C_{\lambda}$ is the union of elementary cells centered at the points of $P \cap p\left(\Omega_{\lambda}\right)$.

Let $\phi_{\lambda}$ be the density of the measure on $\mathfrak{t}^{*}$ which arises by pushing forward the canonical measure on $\Omega_{\lambda}$. The following exercise should be easy for those who know rule 6 for compact groups:

Show that

$$
\phi_{\lambda+\rho}(x)=\sum_{P \cap p\left(\Omega_{\lambda}\right)} m_{\lambda}(\mu) \phi_{\rho}(\mu+x) .
$$

A more difficult and less formal question is:

How is the multiplicity $m_{\lambda}(\mu)$ of a weight $\mu$ in the unirrep $\pi_{\lambda}$ related to the geometry of the set $p^{-1}\left(C_{0}+\mu\right)$ ? 
8.4. Complementary series. The orbit method apparently leaves no place for the complementary series of representations of semisimple groups. Indeed, according to the ideology of the orbit method, the partition of $\mathfrak{g}^{*}$ into coadjoint orbits corresponds to the decomposition of the regular representation into irreducible components. But unirreps of the complementary series by definition do not contribute to this decomposition.

The possible solution to this paradox is based on a remark made in one of the early papers by Gelfand-Naimark. They observed that for non-compact semisimple groups there is a big difference between $L^{1}(G, d g)$ and $L^{2}(G, d g)$ due to the exponential growth of the density of the Haar measure. One of the ingredients of the orbit method is the generalized Fourier transform $\left(2.3 .4^{\prime}\right)$ from the space of functions on $G$ to the space of functions on $\mathfrak{g}^{*}$, which is the composition of two maps:

1. The map from functions on $G$ to functions on $\mathfrak{g}: f \mapsto \phi: \phi(X)=$ $j(X) f(\exp X)$, where $j(X)=\sqrt{\frac{d(\exp X)}{d X}}$;

2. The usual Fourier transform which sends functions on $\mathfrak{g}$ to functions on $\mathfrak{g}^{*}$.

The image of $L^{2}(G, d g)$ under the generalized Fourier transform consists of square integrable functions (at least if we consider functions with support in the domain $\mathcal{E}$ where the exponential map is one-to-one). But the image of $L^{1}(G, d g)$ consists of much nicer functions which admit an analytic continuation from $\mathfrak{g}^{*}$ to some strip in $\mathfrak{g}_{\mathbb{C}}^{*}$. So, one can try to associate complementary series of unirreps with $G$ orbits which lie inside this strip and are invariant under the complex conjugation. One can check that in the simplest case $G=S L(2, \mathbb{R})$ this approach leads to the correct integral formula for the (distributional) character of a representation of the complementary series.

I believe that the problem deserves further investigation.

8.5. Finite groups. Let $G_{n}(K)$ be the group of all upper triangular matrices with elements from some field $K$ and with units on the main diagonal. The case $K=\mathbb{R}$ was one of the main examples illustrating the orbit method in [K1]. It turns out that the case of a finite field $\mathbb{F}_{q}$ (when $G_{n}\left(\mathbb{F}_{q}\right)$ is a finite nilpotent group of order $q^{\frac{n(n-1)}{2}}$ ) is also very interesting (see $[\mathrm{K} 6],[\mathrm{KM}]$ ).

We are interested in the asymptotic properties of harmonic analysis on $G_{n}\left(\mathbb{F}_{q}\right)$ when $q$ is fixed and $n$ goes to infinity. In particular I want to advertise here once again some principal questions:

1. What are the asymptotics of the number of coadjoint orbits for $G_{n}$ ?

2. Can one describe the "generic" or "typical" coadjoint orbit?

3. More generally, which characteristics of orbits and representations can one deal with for the groups of "very large matrices", say, of order $10^{10}$ (or even 20) over a finite field? (Note that the simplest numerical questions about these groups are outside the capability of modern computers.)

Of course, these questions make sense not only for triangular groups and their analogs (unipotent radicals of classical groups). For instance, one can try to find the answer for $G L\left(n, \mathbb{F}_{q}\right)$, using the results from $[\mathrm{Z}]$.

8.6. Infinite dimensional groups. The most intriguing and important question is related to the Virasoro-Bott group. It can be formulated as follows:

How can one explain the (rather complicated) structure of discrete series of unirreps of Vir in terms of coadjoint orbits? 
Note that the very interesting paper [AS] gives an answer to this question, though it is written on the physical level of accuracy and needs a translation into mathematical language.

I leave it to the reader to formulate (and solve) other questions concerning the application of the orbit method to infinite dimensional groups.

\section{REFERENCES}

[AB] Atiyah M.F. and Bott R., The Yang-Mills equations over Riemann surfaces, Phil. Trans. Roy. Soc. A 308 (1982), 523-615. MR 85k:14006

[AG] Arnold V.I. and Givental A.B., Symplectic geometry, Encyclopaedia of Mathematical Sciences, vol. 4, Dynamical systems IV, Springer, 1990, pp. 1-136. MR 88b:58044

[AK] Auslander L. and Kostant B., Polarization and unitary representations of solvable Lie groups, Invent. Math. 14 (1971), 255-354. MR 45:2092

[AS] Alekseev A. and Shatashvili S., Path integral quantization of the coadjoint orbit of the Virasoro group and 2d gravity, Nucl. Phys. B323 (1989), 719-733. MR 90g:22028

[B] Brown I.D., Dual topology of a nilpotent Lie group, Ann. Sci. École Sup. Norm. 6 (1973), 407-411. MR 50:4813

[Ba] Bargmann V., Irreducible unitary representations of the Lorentz group, Ann. of Math. 48 (1947), 568-640. MR 9:133a

[Bu] Busyatskaya I.K., Representations of exponential Lie groups, Funct. Anal. and Appl. 7, No 2 (1973), 151-152.

[BCD] Bernat P., Conze N., Duflo M. et al., Représentations des groupes de Lie résolubles, Dunod, Paris, 1972, pp. 1-272. MR 56:3183

[BK] Brylinski R.K. and Kostant B., Minimal representations, geometric quantization and unitarity, Proc. Natl. Acad. Sci. USA 91 (1994), 6026-6029. MR 95d:58059; Geometric quantization and holomorphic half-form models of unitary minimal representations, I, II (to appear).

[D1] Dixmier J., Sur les représentation unitaires des groupes de Lie nilpotents, III, Canad. J. Math. 10 (1958), 321-348. MR 20:1929

[D2] Dixmier J., Enveloping algebras, North Holland, Amsterdam, 1977. MR 58:16803b

[De] Devaney R.L., An Introduction to Chaotic Dynamical Systems, Addison-Wesley, 1987. MR 91a:58114

[Du1] Duflo M., Fundamental-series representations of a semisimple Lie group, Funct. Anal. and Appl. 4 (1970), 122-126. MR 45:6975

[Du2] Duflo M., Opérateurs différentiels bi-invariants sur un groupe de Lie, Ann. Sci. École Norm. Sup. IV 10 Ser. 3 (1977), 265-283. MR 56:3188

[Du3] Duflo M., Théorie de Mackey pour les groupes de Lie algèbriques, Acta Math. 149 (1982), 153-213. MR 85h:22022

[DH] Duistermaat J.J. and Heckmann G.J., On the variation in the cohomology of the symplectic form of the reduce phase space, Invent. Math. 69 (1982), 259-268. MR 84h:58051a

[DKN] Dubrovin B.A., Krichever I.M. and Novikov S.P., Integrable systems. I, Encyclopaedia of Mathematical Sciences, vol. 4, Dynamical systems IV, Springer, 1990, pp. 173-280. MR 87k:58112

[EFK] Etingof P., Frenkel I.B. and Kirillov A., Jr, Spherical functions on affine Lie groups, Duke Math. Jour. 80, No 1 (1995), 59-90. MR 97e:22018

[EKJ] Etingof P. and Kirillov A., Jr, The unified representation-theoretic approach to special functions, Funct. Anal. and Appl. 28 (1994), 91-94. MR 95h:33010

[F] Fell J.M.G., Weak containment and induced representations of groups, Canad. J. Math. 14 (1962), 237-268. MR 27:242

[Fr] Frenkel I.B., Orbital theory for affine Lie algebras, Invent. Math. 77 (1984), 301-352. MR 86d:17014

[FR] Fock V.V. and Rosly A. A., Flat connections and polyubles, Theoret. and Math. Phys. 95, no. 2 (1993), 526-534. MR 95a:58019

[G] Gelfand I.M., Collected papers, vol. II, Springer, 1988. MR 89k:01042b

[Gi] Ginzburg V.A., The method of orbits in the representation theory of complex Lie groups, Funct. Anal. and Appl. 15 (1981), 18-28. MR 83f:22011; $\mathfrak{g}$-modules, Springer's representations and bivariant Chern classes, Adv. in Math. 61 (1986), 1-48. 
[GLS] Guillemin V., Lerman E. and Sternberg S., Symplectic fibrations and multiplicity diagrams, Cambridge Univ. Press, 1996. MR 98d:58074

[GS] Guillemin V. and Sternberg S., Quantization and multiplicities of group representations, Invent. Math. 67 (1982), 515-538. MR 83m:58040; Symplectic techniques in physics, Cambridge Univ. Press, 1994. MR 86f:58054; MR 91d:58073

[H] Heckman G.J., Projections of orbits and asymptotic behavior of multiplicities for compact connected Lie groups, Invent. Math. 67 (1983), 333-356. MR 84d:22019

[Ho1] Howe R., On Frobenius reciprocity for unipotent algebraic groups over $\mathbb{Q}$, Amer. J. Math. 93 (1971), 163-172. MR 43:7556

[Ho2] Howe R., Reciprocity laws in the theory of dual pairs, Representation Theory of Reductive Groups (P. Trombi, ed.), Progress in Math., vol. 40, Birkhäuser, Boston, 1983. MR 85k:22033

[I] Isaacs I.M. and Karagueuzian D., Conjugacy in Group of Upper Triangular Matrices, J. of Algebra 202 (1998), 704-711. MR 99g:20021

[Is] Ismagilov R.S., Representations of infinite dimensional groups, Translations of Mathematical Monographs, vol. 152, AMS, 1996. MR 97g:22014

[K1] Kirillov A.A., Unitary representations of nilpotent Lie groups, Russian Math. Surveys 17 (1962), 57-110. MR 25:5396

[K2] Kirillov A.A., Elements of the theory of representations, Springer, 1976 (Russian editions 1972, 1978). MR 54:447

[K3] Kirillov A.A., Introduction to the theory of representations and noncommutative harmonic analysis, Encyclopaedia of Mathematical Sciences, vol. 22 Representation theory and noncommutative harmonic analysis I, Springer, 1994, pp. 1-156. CMP 95:06

[K4] Kirillov A.A. ( Adams J., Kleppner A., Li J.-S., Lipsman R., Rosenberg J., eds), Introduction to the orbit method. I, II., Progress in Math. 145 (1992). MR 94i:22042

[K5] Kirillov A.A., Geometric quantization, Encyclopaedia of Mathematical Sciences, vol. 4 Dynamical systems, Springer, 1990 (1997, the second edition), pp. 137-172. MR 87k:58104

[K6] Kirillov A.A., Variations on the triangular theme, AMS Translations, vol. 169, AMS, 1995, pp. 43-74. MR 97a:20072

[K7] Kirillov A.A., The characters of unitary representations of Lie groups, Funct. Anal. and Appl. 2, No 2 (1968), 133-146. MR 38:4615

[K8] Kirillov A.A., Geometric approach to Discrete Series of Unirreps for Vir, Comm. Pures Appl. Math. 77 (1998), 735-746. MR 99h:17027

[Ka] Kac V., Infinite dimensional Lie algebras, Third edition, Cambridge University Press, 1990. MR 92k: 17038

[Ko1] Kostant B., Quantization and unitary representations, Lecture Notes in Math., vol. 170, Springer, 1970, pp. 87-208. MR 52:5892; Graded manifolds, graded Lie theory and prequantization, Lecture Notes in Math., vol. 570, Springer, 1970, pp. 177-306. MR 58:28326

[Ko2] Kostant B., On the existence and irreducibility of certain series of representations, Lie groups and their representations, Proceedings of the Summer School of the Bolyai Janos Math. Soc., I.M. Gelfand, Editor, John Wiley and Sons, 1975, pp. 231-331. MR 53:3206

[Ko3] Kostant B., The solution to a generalized Toda lattice and representation theory, Adv. in Math. 34 (1979), 195-338. MR 82F:58045

[KK] Kirillov A.A. and Kontsevich M.L., The growth of the Lie algebra generated by two generic vector fields on the real line, Vestnik Mosk. Univ. Ser. 14 (1983), 15-20. MR 86d:17012

[KKM] Kirillov A.A., Kontsevich M.L. and Molev A.I., Algebras of intermediate growth, Selecta Math. Sov. 9 (1990), 155-169. MR 85e:17011

[KM] Kirillov A.A. and Melnikov A., On a remarkable sequence of polynomials, Proceedings of the Franco-Belge Colloquium, Reims 1995, Collection S.M.F., 1997, pp. 35-42. CMP 98:07

[Kon] Kontsevich M., Formality conjecture. Deformation theory and symplectic geometry, Math. Phys. Stud., vol. 20, Kluwer Acad. Publ., Dordrecht, 1996, pp. 139-156. MR 98m:58044

[KV] Kashiwara M. and Vergne M., The Campbell-Hausdorff formula and invariant hyperfunctions, Invent. Math. 47 (1978), 249-272. MR 58:11232

[KY] Kirillov A.A. and Yuriev D.V., Kähler geometry of the infinite-dimensional homogeneous space $M=\operatorname{Diff}+\left(S^{1}\right) / \operatorname{Rot}\left(S^{1}\right)$, Funct. Anal. and Appl. 21 (1987), 284-294. MR 89c:22032

[LL] Leptin H. and Ludwig J., Unitary representation theory of exponential Lie groups, Walter de Gruyter \& Co., 1994, pp. X + 200. MR 96e:22001 
[LW] Lu J.H. and Weinstein A., Poisson-Lie groups, dressing transformations and Bruhat decompositions, J. Diff. Geom. 31 (1990), 501-526. MR 91c:22012

[M] Moore C.C., Decomposition of unitary representations defined by a discrete subgroup of nilpotent groups, Ann. of Math. 82 (1965), 146-182. MR 31:5928

[N] Neretin Yu.A., Categories of Symmetries and Infinite-dimensional Groups, LMS Monographs New Series 16, Oxford University Press, 1996, pp. xiv + 417. MR 98b:22003

[P1] Pukanszky L., On the theory of exponential groups, Trans. Amer. Math. Soc. 126 (1967), 487-507. MR 35:301

[P2] Pukanszky L., Unitary representations of solvable Lie groups, Ann. Sci. Ec. Norm. Sup. IV, Ser. 4 (1971), 457-608. MR 55:12866

[PS] Pressley A. and Segal G., Loop groups, Clarendon Press, Oxford, 1986. MR 88i:22049

[R] Rossmann W., Kirillov's character formula for reductive Lie groups, Invent. Math. 48 (1978), 207-220. MR 81g:22012

[RS] Reiman A.G. and Semenov-Tian-Shanskii M.A., Group-theoretic methods in the theory of integrable systems, Encyclopaedia of Mathematical Sciences, vol. 16, Dynamical systems VII, Springer, 1994, pp. 116-262. MR 94h:58069

[S] Souriau J.-M., Structure des systèmes dynamiques, Dunod, 1970. MR 41:4866

[Sh] Shchepochkina I.M., On representations of solvable Lie groups, Funct. Anal. and Appl. 11 (1977), 93-94; Orbit method for the restriction-induction problems for normal subgroup of a solvable Lie group, C.R. de l'Acad. bulgare des Sci. 33 (1980), 1039-1042. MR 82m:22012

[So] Soibelman Ya., Orbit method for the algebra of functions on quantum groups and coherent states. I., Int. Math. Res. Notices 6 (1993), 151-163. MR 94c:17033

[V] Vogan D., Jr, Associated varieties and unipotent representations, Progr. Math., vol. 101, Birkhäuser, Boston, 1991. MR 93k:22012; Unitary representations of reductive Lie groups, Princeton Univ. Press, 1987. MR 89g:22024

[W1] Witten E., Non-abelian bosonization in two dimensions, Commun. Math. Phys. 92 (1984), 455-490 MR 86g:81104; Quantum field theory and the Jones polynomial, Commun. Math. Phys. 121 (1989), 351-399. MR 90h:57009

[W2] Witten E., On quantum gauge theories in two dimensions, Commun. Math. Phys. 141 (1991), 153-209. MR 93i:58164

[WD] Wildberger N.J., On a relationship between adjoint orbits and conjugacy classes of a Lie group, Canad. Math. Bull. 33 (3) (1990), 297-304 MR 92a:22010; Dooley A.H. and Wildberger N.J., Harmonic analysis and the global exponential map for compact Lie groups, Funct. Anal. and Appl. 27 (1993), 25-32. MR 94e:22032

[Z] Zelevinsky A.V., Representations of finite classical groups, In: Lecture Notes in Math., vol. 869, Springer, 1981, pp. 1 -184. MR 83k:20017

[Za] Zagier D., Values of zeta functions and their applications, In: First European Congress of Mathematics, Vol. II (Paris, 1992), Birkhäuser, Basel, 1994, pp. 497-512. MR 96k:11110

Department of Mathematics, The University of Pennsylvania, Philadelphia, PA 19104-6395

E-mail address: kirillov@math.upenn.edu

Institute for Problems of Information Transmission, Russian Academy of Sciences, B. Karetny 19, Moscow 101 477, GSP-4, Russia 